I $\mathrm{BL}--23302$

DE87 011212

\title{
A Measurement of the Low Frequency Spectrum of the Cosmic Microwave Background Radiaion
}

\author{
Steven M. Levin \\ (PhD Dissertation)
}

Lawrence Berkeley Laboratory

University of California

Berkeley, California 94720

\author{
Committee: $\quad$ P. Buford Price, Co-Chair \\ George F. Smoot, Co-Chair \\ Marc Davis \\ William J. Welch
}

\section{DISCLAIMER}

This report was prepared as an account of work spensored by an agency of the United States Government. Neither the United States Government n' employees, makes any warranty, express or implied, or assumes any legal liability or responsi. bility for the accuracy, completeness, or usefulness of any information, apparatus, product, or process disclosed, or represents that its use would not infringe privately owned rights. Reference herein to any specific commercial product, process, or service by trade name, trademark. manufacturer, or otherwise does not necessarily constitute or imply its endorsement, recommendation, or favoring by the United States Government or any agency thereof. The views and opinions of authors expressed herein do not necessarily state or reflect those of the United States Government or any agency thereof.

\section{MASTER}

IISTRBBUTION OF THIS DOCUMENT IS UNLIMITEO 
A Low-Frequency Measurement of the Spectrum of the Cosmic Microwave Background Radiation

Steven Mark Levin

\begin{abstract}
As part of a larger effort to measure the spectrum of the Cosmic Background Radiation (CBR) at low frequencies, the intensity of the CBR has been measured at a frequency of $1.410 \mathrm{GHz}$. The measurement was made by comparing the power received from the sky with the power received from a specially designed cooled calibration target with known properties. Sources of radiation other than the CBR were then identified and subtracted to calculate the antenna temperature of the $\mathrm{CBR}$ at $1.410 \mathrm{GHz}$.

The instrument used to measure the CBR was a total-power microwave radiometer with a $25 \mathrm{MHz}$ bandwidth centered at 1.410 GHz. The radiometer had a noise temperature of $80 \mathrm{~K}$, and sufficient data were taken that radiometer noise did not contribute significantly to the total measurement error. The sources of error were predominantly systematic in nature, and the largest error was due to uncertainty in the reflection characteristics of the coldload calibrator. Identification and subtraction of signals from the Galaxy $(0.7 \mathrm{~K})$ and the Earth's atmosphere $(0.8 \mathrm{~K})$ were also significant parts of the data reduction and error analysis.
\end{abstract}


The brightness temperature of the Cosmic Background Radiation at $1.410 \mathrm{GHz}$ is $2.22 \pm 0.55$ Kelvin. The spectrum of the CBR, as determined by this measurement and other published results, is consistent with a blackbody spectrum of temperature $2.741 \pm 0.016$. Constraints on the amount by which the CBR spectrum deviates from Planck spectrum are used to place limits on energy releases early in the history of the universe.

The experimental error could be reduced by changes in the instrument and measurement procedure, and several possible improvements are described. In particular, alternative methods for reducing reflection effects from the cold-load calibrator are described, as are possible improvements in the measurement of the galactic contribution. 
$\begin{array}{lll}\text { Chapter I: } & \text { Introduction } & 1\end{array}$

Chapter II: $\quad$ Concept of Experiment 9

$\begin{array}{lll}\text { Chapter III: } & \text { The Instrument }\end{array}$

Chapter IV: The Measurement 32

Chapter V: $\quad$ Analysis $\quad 38$

Section i: $G\left(S_{\text {zenith }}-S_{\text {load }}\right) \quad 40$

Section ii: Absolute Reference Load 43

Section iii: $T_{\text {galaxy }} \quad 58$

Section iv: $\Delta \mathrm{T}_{\text {offset }} \quad 64$

Section v: $T_{\text {ground }} \quad 67$

Section vi: $T_{\text {atmosphere }} \quad 69$

$\begin{array}{lll}\text { Chapter VI: } & \text { Results and Conclusions }\end{array}$

$\begin{array}{lll}\text { Chapter VII: Interpretation } & 74\end{array}$

$\begin{array}{lll}\text { Chapter VIII: } & \text { Future Work } & 91\end{array}$

$\begin{array}{lll}\text { Chapter IX: } & \text { Acknowledgements }\end{array}$

$\begin{array}{lll}\text { Chapter X: } & \text { References } & 97\end{array}$

Appendix A: The 2-18 GHz Radiometers

Appendix B: The Atmosphere

Appendix C: The Antenna 
Figure 1: $T_{C B R}$ as a function of time Page 4

Figure 2: Concept of Experiment Page 10

Figure 3: Atmospheric emission at sea level Page 12

Figure 4: Atmospheric and Galactic emission Page 13

Figure 5: Schematic of spectrum experiment Page 16

Figure 6: The observing site Page 17

Figure 7: The cold-load calibrator Page 20

Figure 8: The ambient-temperature calibrator Page 22

Figure 9: Schematic diagram of the radiometer Page 25

Figure 10: Physical layout of the electronics Page 26

Figure 11: Return loss from the LHe dewar Page 33

Figure 12: Gain as a function of time Page 42

Figure 13: Coherent reflection effects Page 44

Figure 14: Apparatus used for measuring return loss Page 47

Figure 15: Schematic of sliding short apparatus Page 49

Figure 16: Radiometer output vs. reflection phase Page 50

Figure 17: LHe dewar mockup Page 53

Figure 18: Reflection from dewar mockup Page 54

Figure 19: Galactic emission measurement Page 61

Figure 20: Predicted galactic emission Page 62

Figure 21: Gain pattern of the $1.4 \mathrm{GHz}$ antenna Page 68

Figure 22: CBR spectrum measurements 
Figure 23: Contours of constant $\chi^{2}$ for model fits

Page 83

Figure 24: Limits on fractional energy release

Page 84

Figure 25: Sample CBR spectral distortions

Page 89

Figure 26: Model distorted spectrum from dust grains Page 90

Figure A1: Schematic of 2-18 GHz radiometers (1984) Page A2

Figure A2: The 2-18 $\mathrm{GHz}$ radiometer

Page A4

Figure A3: Conical ground shield

Page A.6

Figure A4: Schematic of 2-18 GHz radiometers (1986) Page A9

Figure A5: The 2-8 GHz radiometer and cart in 1986 Page A11

\section{Tables}

Table 1: Radiometer characteristics

Page 29

Table 2: Sky temperature minus LHe temperature Page 41

Table 3: Power reflection coefficients

Page 46

Table 4 Phase variation of reflected signal

Page 52

Table 5 Recent measurements of TCBR

Page 73

Table 6 Distortion parameter fits

Page 82

Table 7 Preliminary data from Lange et al.

Page 86

Table 8 Distortion parameter fits with new data

Page 87 
There are as many reasons for doing physics as there are physicists. For most, however, an important reason is the desire to know "what makes things tick," to find simplicity underlying the behavior of apparently complex objects. There can be no object more complex than the universe itself, and the model I am about to discuss is as simple as it is audacious. The Hot Big Bang model of cosmology, so well accepted that it's become known as the "standard model," is based on the idea that long ago the universe was very much hotter and denser than it is today. This cosmic fireball expanded and cooled, and over the course of 10 to 20 billion years, evolved into the present universe.

One of the basic assumptions, elevated to the level of a principle, of modern cosmology is the Cosmological Principle, which states that on a large scale the universe is spatially homogeneous and isotropic. This dictates that it can be described by the Robertson-Walker metric:

$$
d s^{2}=d t^{2}-R^{2}(t)\left[d r^{2}\left(1-k r^{2}\right)^{-1}+r^{2} d \theta^{2}+\left(r^{2} \sin ^{2} \theta\right) d \phi^{2}\right]
$$

Here $\mathbf{k}$, which is commonly normalized to 1,0 , or -1 , parametrizes the curvature of space, and $R(t)$, called the cosmic scale factor, is proportional to the distance separating any two points in space as it evolves with time.

An immediate consequence of the Cosmological Principle is Hubble's Law, discovered by Hubble (1929) in redshift measurements of 
galaxies. Hubble's Law states that the recessional velocity of an object is directly proportional to the distance to that object. This can be written as

$$
H(t)=\frac{1}{R(t)} \frac{d R}{d t}
$$

where $H(t)$, known as the Hubble parameter, is constant in space at any particular epoch. Current estimates for $\mathrm{H}(\mathrm{t})$, called $\mathrm{H}_{0}$ when referring to the present epoch, range from 50 to 100 km-sec-1-Mpc-1 (Sandage and Tammann, 1976, de Vaucouleurs, 1979). By extrapolating back in time the paths of the galaxies we see receding, we can infer a time, roughly 10 to 20 billion years ago, when the separation between any two objects was essentially zero. Conventionally, this point is taken as the origin for our time axis, and the expansion which followed is called the Big Bang.

The radiation left from the hot Big Bang is called the Cosmic Background Radiation (CBR). The CBR is one of the few probes available to us to study the early history of the universe. For the most part, CBR photons that reach us today have traveled undisturbed for 10 to 20 billion years, from a time when the universe was 1000 times smaller and hotter than it is today. The CBR taken as a whole may carry information from times as early as a year or two after the Big Bang. The effort to decipher that information has been underway since 1964, when Penzias and Wilson first discovered the CBR (Penzias and Wilson 1965). 
Soon after the Big Bang, when the universe was much denser and hotter than it is today, matter and radiation interacted so rapidly and both were so energetic that virtually every kind of particle was rapidly being created and destroyed in a dense, high temperature medium. As the universe expanded and cooled, particles began to "freeze out".

Figure 1 shows the CBR temperature as a function of time. When the temperature dropped below the energy level required for pair production of muons, the rate of reactions producing muons decreased dramatically. Since the muons continued to annihilate and decay, there were virtually no muons shortly after the temperature dropped below $10^{12} \mathrm{~K}$. Similarly, the electron density dropped after $\sim 1010 \mathrm{~K}$, as electrons and positrons annihilated until their number was so low that the slight excess of electrons over positrons predominated.

After the temperature dropped below about $10^{9} \mathrm{~K}$, the photon energy was low enough that deuterium formed faster than the radiation broke it apart. The deuterium therefore stayed around long enough to fuse, and helium nucleosynthesis took place. As the universe continued to expand and cool, densities got low enough that, while the photons still interacted with the matter, interactions with more than one particle became rare, and ordinary Compton scattering became the dominant interaction mechanism. (This 


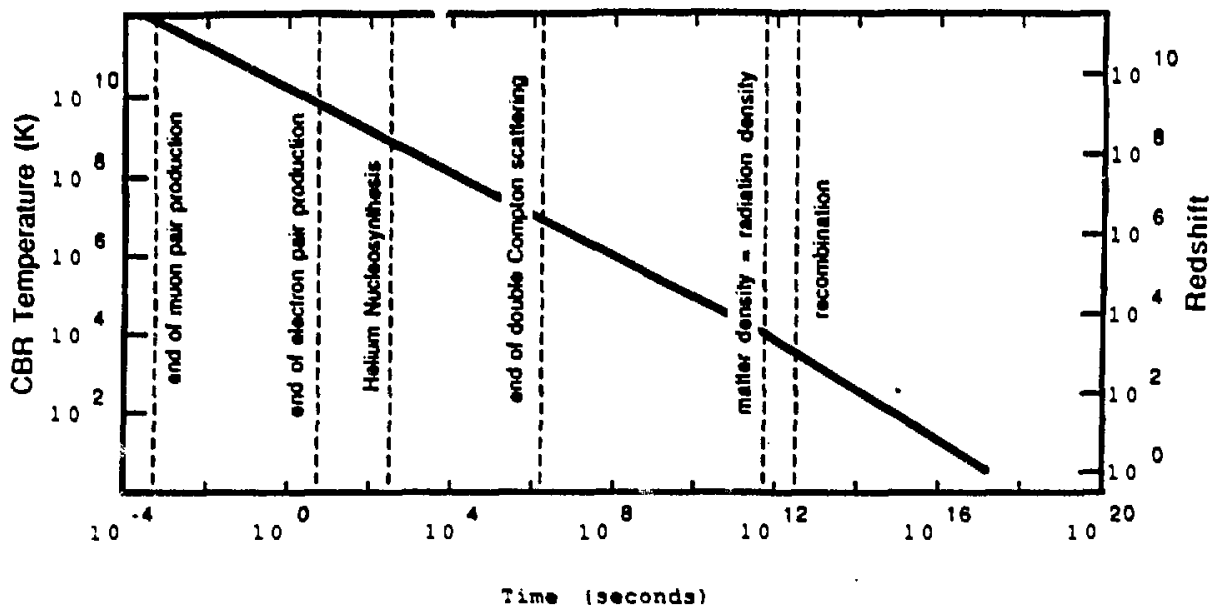

Figure 1. The temperature of the Cosmic Background Radiation as a function of time. 
important point will be discussed further in the Chapter VII.)

For redshifts greater than a few thousand, the universe was radiation dominated; that is, the energy in the CBR was. greater than the energy in the matter. ïventually, as the cooling continued and the mass-energy of the particles became important by comparison with their temperature, the universe became matter-dominated. Shortly afterwards, the temperature dropped below that required to ionize hydrogen, and the protons and electrons combined to form neutral hydrogen atoms. This event (called "recombination") dramatically changed the relationship of the CBR with the rest of the universe, since neutral hydrogen atoms have a much lower cross section for interacting with light than do free electrons. The ratio of the crosssection for Rayleigh scattering of photons by neutral hydrogen to the cross section for Compton scattering by free electrons is approximately $\left(v / v_{0}\right)^{4}$, where $v_{0}$ is $-3 \times 10^{6} \mathrm{GHz}$ and $v$ for the peak of the CBR at that time was $3 \times 10^{5} \mathrm{GHz}$. The mean free path for photons became larger than $10^{10}$ light years, the universe was transparent, and the CBR trayeled virtually undisturbed for the remaining $10-20$ billion years.

The description above leads to the conclusion that when the CBR last interacted with matter it had a Planckian spectrum. For a Planckian spectrum the density of photons is proportional to $T^{3}$, and the energy density is proportional to $T^{4}$, where $T$ is the temperature. In a 
uniformly expanding universe, the photon density drops proportionally to the cube of the expansion, $R^{3}$, and the wavelength of the photons increases proportionally to $R$, so that the energy per photon decreases linearly in $R$. Hence the energy density goes as $R^{-4}$, so the expansion produces another Planckian spectrum, with temperature inversely proportional to $R$. Thus the Standard Model predicts that today the CBrian should have a Planckian spectrum, and indeed all measurements of the spectrum to date are consistent with such a spectrum.

As well as a Planckian spectrum for the CBR, the foregoing description predicts a universe consisting of natter spread evenly in all directions, with no clumps, no light sources, no exchanges of energy between the matter and the radiation, and no galaxies, stars, or planets. Clearly, the actual universe is more complex. Most such complexities would have left fingerprints on the CBR. In particular, any interaction of matter with the radiation would affect the spectrum of the CBR. If such interaction took place early enough (before a redshift of $\sim 10^{6}$ ), new photons were produced at low frequencies, Compton scattering redistributed their energies, and the result was a Planckian spectrum for the $C B R$, at an increased temperature. For interactions at later times, when the density was lower and the photon production rate not as high, the spectrum of the CBR would be distorted to something other than a purely Planckian spectrum. By looking for such distortions, one can study 
the early history of the universe.

In the short time since the discovery of the CBR, there have been several attempts to determine its spectrum. Immediately after the discovery of the CBR by Penzias and Wilson in 1965, numerous measurements were made of its intensity at various frequencies, ranging from $400 \mathrm{MHz}$ to $90 \mathrm{GHz}$. These initial measurements were primarily intended to verify the existence of the CBR. Subsequent measurements verified that the CBR spectrum had a roughly Planckian shape by finding the Wien fall-off (Weiss, 1980 and references therein). Still, by the end of the 1970's, a distortion in the CBR spectrum as large as $20 \%$ could have gone undetected.

In the early 1980's, substantial improvements were made in the measurements of the CBR spectrum at frequencies above $100 \mathrm{GHz}$ (Peterson, Richards, and Timusk 1985, Meyer and Jura 1985), and preliminary measurements prompted considerable speculation about the possible distortions and their causes.

In 1978, a group at the Lawrence Berkeley Laboratory and the Space Sciences Laboratory in Berkeley began to consider a remeasurement of the CBR spectrum in the Rayleigh-Jeans region. Simultaneously, an international collaboration of researchers from Italy and the United States was beginning a similar effort. In 1979 the two efforts were combined, and the collaboration made several measurements at frequencies of $2.5,4.75,10,33$, and $90 \mathrm{GHz}$ (Smoot et al 1983, Smoot 
et al 1985a, b) which substantially improved the limits on possible distortions of the CBR spectrum.

I joined the Berkeley group in 1983, and began working on the 2-18 $\mathrm{GHz}$ radiometers (see Appendix $\mathrm{A}$ ), which were developed as an extension of the collaborative project. In 1985, after the completion of the collaborative effort, the Berkeley group began working on two new radiometers, operating at $1.4 \mathrm{GHz}$ and at $3.7 \mathrm{GHz}$. By July of 1986, when we returned to the high-altitude research station where we had made previous measurements, the 2-18 $\mathrm{GHz}$ radiometers had been abandoned, and we made new measurements at 1.4, 3.7, 10, and $90 \mathrm{GHz}$. During the measurements and the analysis which followed, the $1.4 \mathrm{GHz}$ radiometer was my primary responsibility, and it will be the focus of this dissertation. 
The basic concept of the experiment is to compare the microwave signal from the sky with the signal from a low-temperature calibration target with known radiometric properties. After thus determining the signal from the sky, we subtract the signals from all sources other than the CBR to arrive at the intensity of the CBR at $1.4 \mathrm{GHz}$. To make this measurement, we used a radiometer, a device whose output voltage is proportional to the microwave power intercepted by its antenna. The radiometer is discussed in detail in Chapter III.

Microwave signals are measured in antenna temperature, defined by

$$
k T_{A}=P / B
$$

where $k$ is Boltzmann's constant, $P$ is the power received in bandwidth $B$, and $T_{A}$ is the antenna temperature. A blackbody filling the aperture at thermodynamic temperature $T$ will have an antenna temperature of

$$
T_{A}=\frac{x T}{\left(e^{x}-1\right)}=\frac{T_{V}}{\left(e^{T_{V} / T}-1\right)}
$$

where $x=h v / k T, v$ is the frequency, $h$ is Planck's constant, and the antenna temperature of a single photon, $T_{v}=h v / k$, is $0.067 \mathrm{~K}$ at $1.4 \mathrm{GHz}$.

As shown in Figure 2, there are a variety of signals which must be identified or eliminated in order to arrive at the antenna 


\section{Page $10 \quad$ Chapter II: Concept of Experiment}

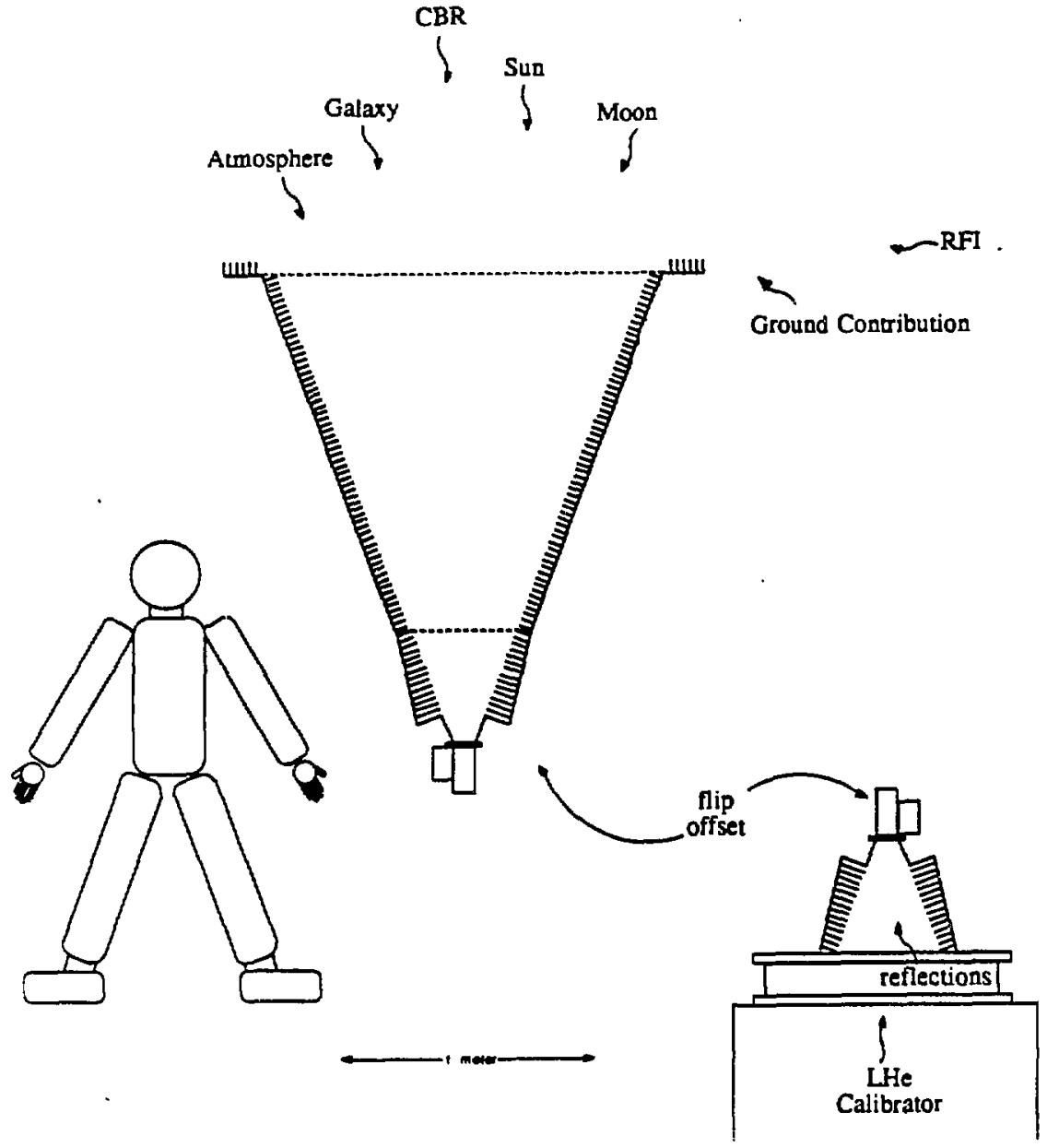

Figure 2. Concept of the experiment. The radiometer alternately is pointed at the sky and at a calibration target. After comparing the signal from the sky with the signal from the target to determine the total power received from the sky, all sources of radiation other than the Cosmic Background Radiation are identified and subtracted. 
temperature of the CBR, including signals from the Sun and the Moon, the Galaxy, the Earth's atmosphere, man-made interference, contributions from the ground, from gravitational stresses, and from the calibration target. Each of these signals will be discussed in more detail in Chapter $V$, but I will note here their major effects on the design of the experiment.

Signals from the Sun and the Moon are eliminated simply by taking data only at night, at times when the Moon is near or below the horizon. Similarly, RF interference and diffracted signals from the ground were reduced by careful design of the antenna (discussed in Chapter III and in Chapter V, Section v) and by choosing a remote site, far from most man-made signals.

The two signals which were most influential in the design of this experiment are the signals from the atmosphere and from the Galaxy. The atmosphere is not perfectly transparent in the microwave region (see Figure 3 ), and its thermodynamic temperature is high, so its antentic iemperative is comparabie to the signal we wish to measure, TCBR. The experiment therefore had to be designed to minimize the atmospheric contribution, and to identify the remaining signal. Choosing a high-altitude site helped to reduce the problem (see Figure 4), but the signal was still significant.

Figure 4, shows that the atmospheric signal is smaller at lower frequencies. The same plot, however, shows that the signal from the 
Page 12 Chapter II: Concept of Experiment

Atmospheric Emission at Sea Level

$3-$

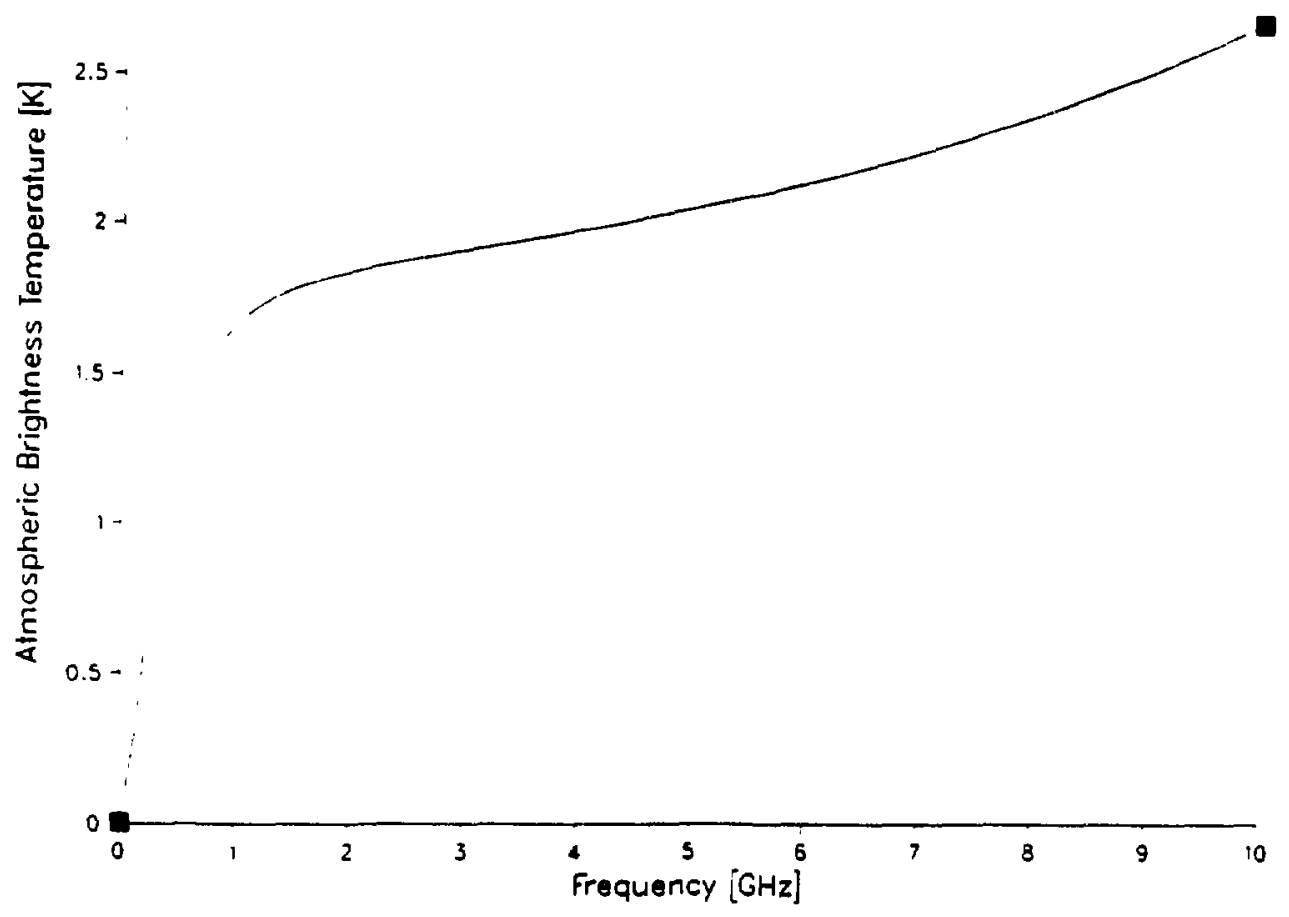

Figure 3. Estimated antenna temperature of the atmosphere as viewed vertically from sea level. (Water vapor content is $8 \mathrm{~mm}$.) 
Atmospheric Emission at 3800 Meters

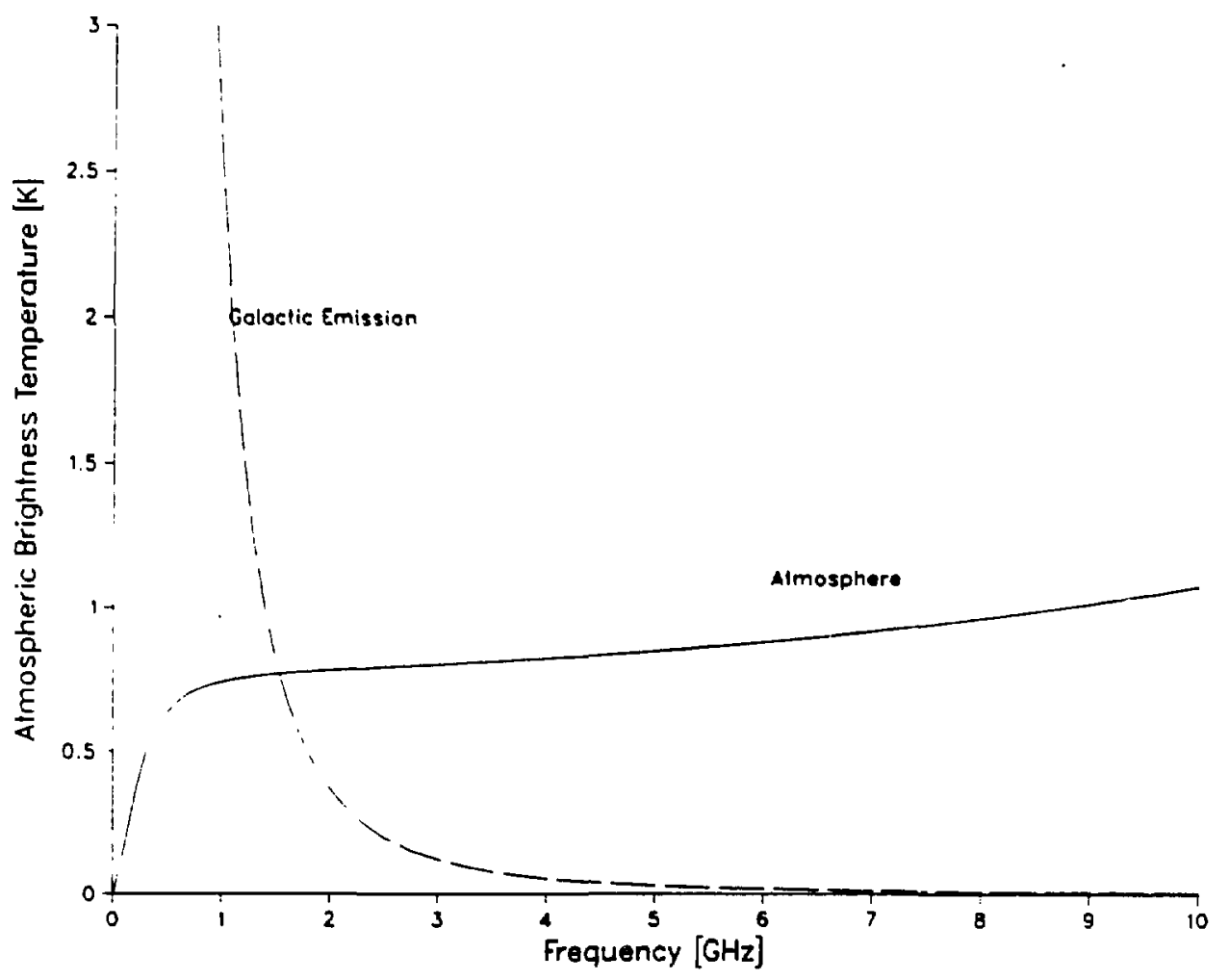

Figure 4. Estimated antenna temperature of the atmosphere as viewed vertically from an altitude of 3800 meters. (Water vapor content is $4 \mathrm{~mm}$.) Typical emission from the Galaxy is also shown. Galactic emission is spatially dependent, ranging from a high 3 times the typical emission shown to a low half as large as the emission depicted here. 
galaxy increases sharply with decreasing frequency. The choice of $1.4 \mathrm{GHz}$ as an operating frequency is a compromise between minimizing the errors caused by the galactic and by the atmospheric contributions, tempered by our desire to observe at lower frequencies, where interesting physics may heretofore have remained undetected.

Despite the choice of site and frequency, both the galactic signal and the atmospheric signal must be measured and subtracted. In measurements at other frequencies, we have measured the atmospheric contribution by tipping the radiometer to various zenith angles, and fitting the resultant signal changes to a model of the atmospheric pathlength traversed by the antenna pattern. Because of the size of the $1.4 \mathrm{GHz}$ radiometer, we could rot easily duplicate this technique. We therefore elected to extrapolate from simultaneous measurements of the atmospheric signal at other frequencies, a method I will describe in more detail later.

Unlike the atmospheric contribution, the galactic signal at $1.4 \mathrm{GHz}$ can be measured directly with our design. The radiometric signal from the galaxy has both a frequency and a spatial dependence, and we used these dependences to estimate the size of the signal. We assumed that $T_{C B R}$ varies slowly with frequency by comparison with the Galaxy's steep power law, and measured the galactic signal by comparing the sky signals at two nearby frequencies, fitting to an 
appropriate power law. Similarly, we used the Galaxy's spatial dependence to good advantage by letting the Earth's rotation bring different parts of the Galaxy overhead, and fitting the resulting profile to a model of the galactic structure. Because such a galactic scan takes a minimum of several hours, we had to calibrate the radiometer with a known noise source many times during the scan. We can also estimate the galactic signal by using some of our precious time over the cold calibration target to measure the signal from the sky at a time when the galactic plane is nearly overhead (so that the signal is large). By comparing this with the signal when observing far from the galactic plane, we can fit our measurements to a model of the spatial structure of the Galaxy. We used this last method as well, and I'll describe the results later. The expected galactic emission affected the experimental design mainly in that, in preparation for galactic scans, we fitted the radiometer with a removable noise snurce capable of being turned on and off by computer, and designed the instrument to be capable of changing frequency slightly $(165 \mathrm{MHz})$ in an automated fashion.

As well as the radiometer, the experimental design had to include the equipment needed to run the instrument and record the data. As shown in Figure 5, a variety of support equipment is needed to run the radiometer. The radiometer is powered by two pairs of 12 volt batteries, each connected in series to produce a 24 volt power source. The power for the temperature control circuits comes from one set of 


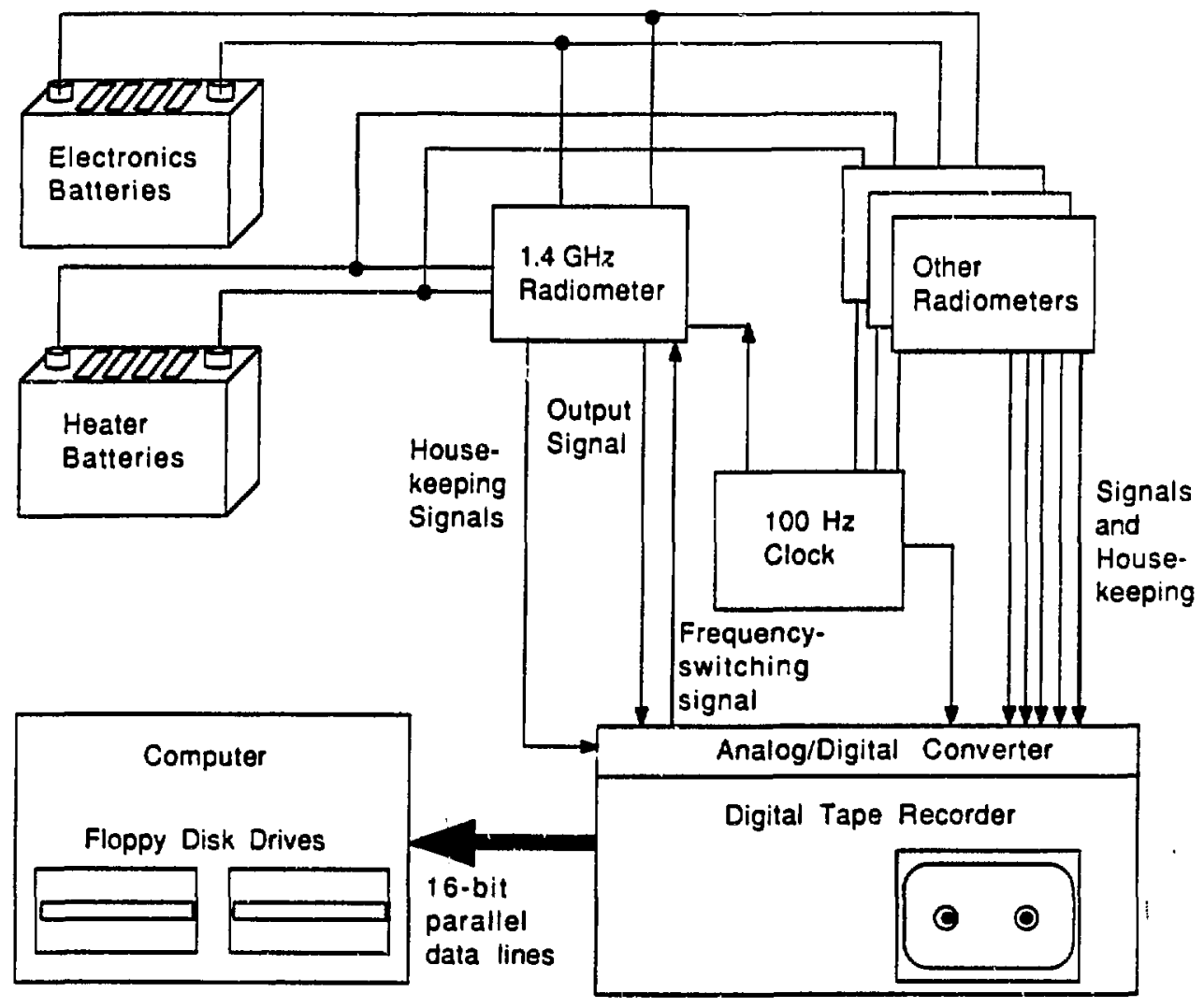

Figure 5. Schematic diagram of the spectrum experiment. 


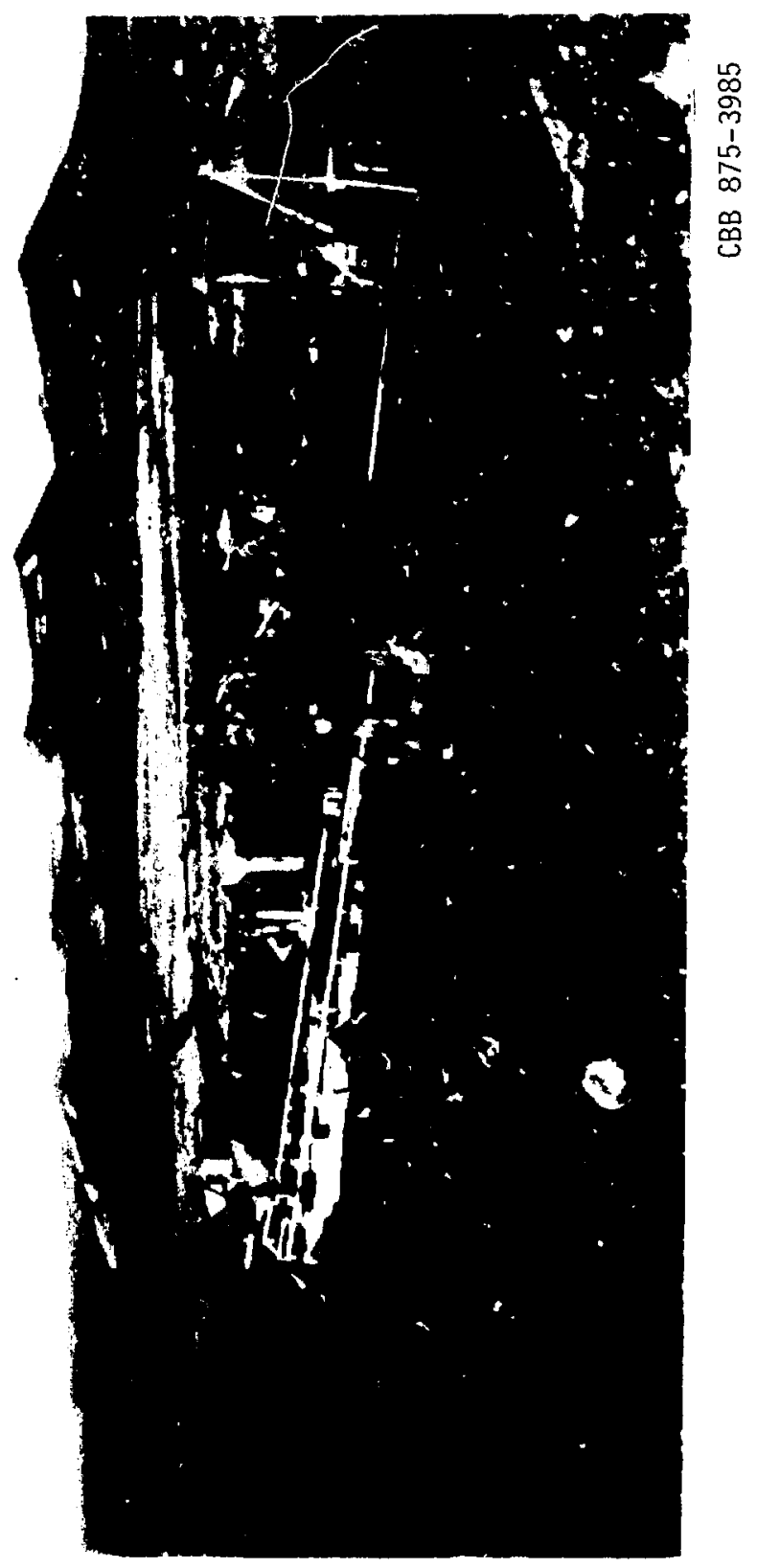

Figure 6a. 
Chapter II: Concept of Experiment

Page 18

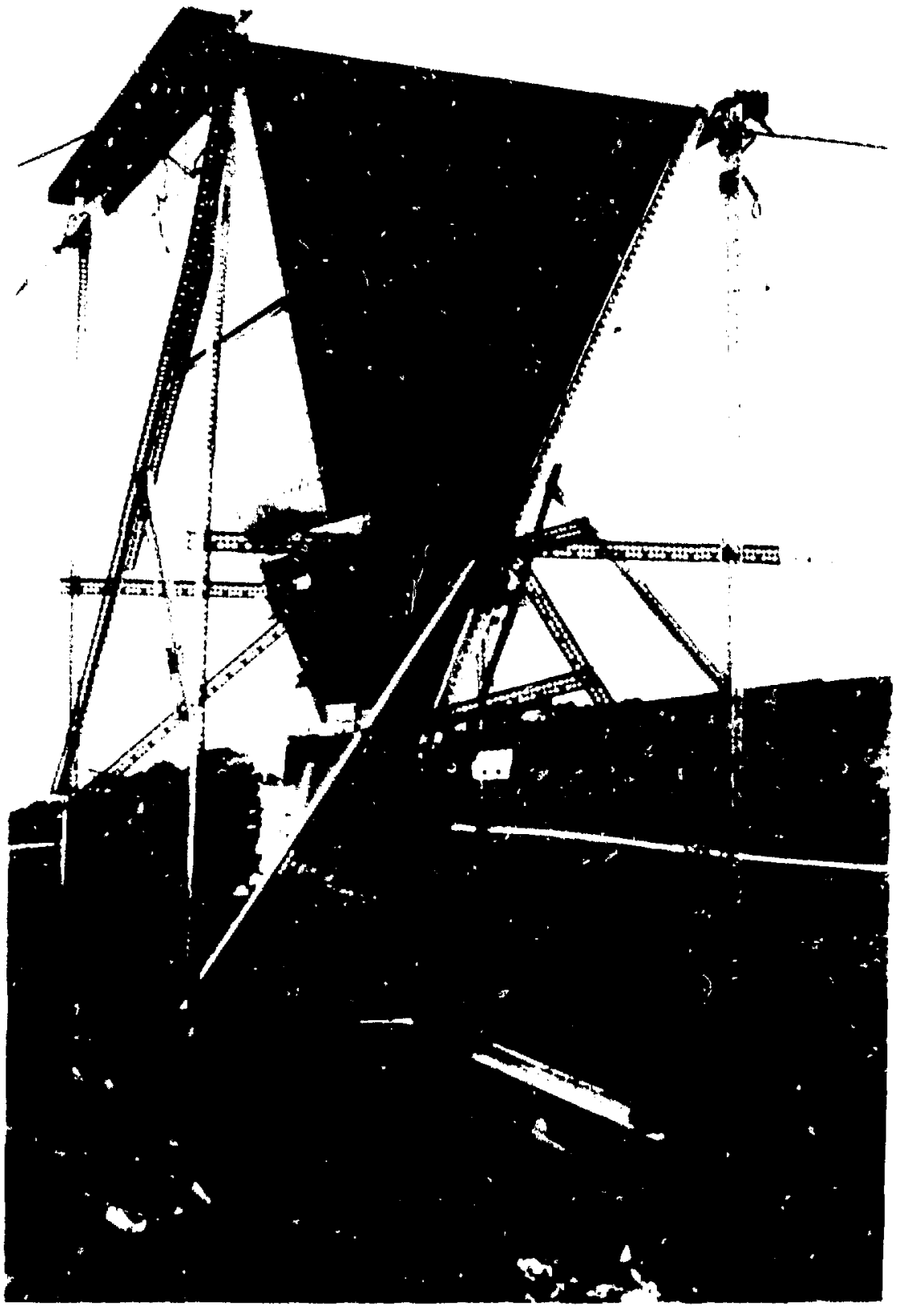

CAB $875-3987$

Figure $6 \mathrm{~b}$. The $1.4 \mathrm{GHz}$ radiometer at the observing site. 
batteries, and the power for the radiometer electronics comes from the other set. By keeping the two isolated, we eliminate any possibility of cross talk between the heaters which maintain a constant temperature and the amplifiers which produce the signal. The thermal control circuit was also designed to respond to temperature changes proportionally, so that there were no large current surges. The batteries are kept charged by a power supply (Electro Products Laboratory Modei NFB) powered from the availabie $110 \mathrm{~V}$ lines.

Once the radiometer has collected and araplified a signal, the signal still must be recorded for analysis. We recorded the radiometer olitput, as well as the signals from the thermal controller and from switches used to manually indicate the target being viewed, on magnetic tape with a digital tape recorder. A computer was also used to monitor the process in real time, and store backup data on floppy disks.

The observing site is shown in Figure 6. The radionseters (except the 3.7 $\mathrm{GHz}$ radiometer, which is free-standing) were rolled along the rails to view the LHe target, which was below glound level in the center of the rails. When not viewing the LHe target, the radiometers were rolled to the end of the rails, so that they would not interfere with other measurements.

The calibration target was kept cold with liquid helium, so that its 

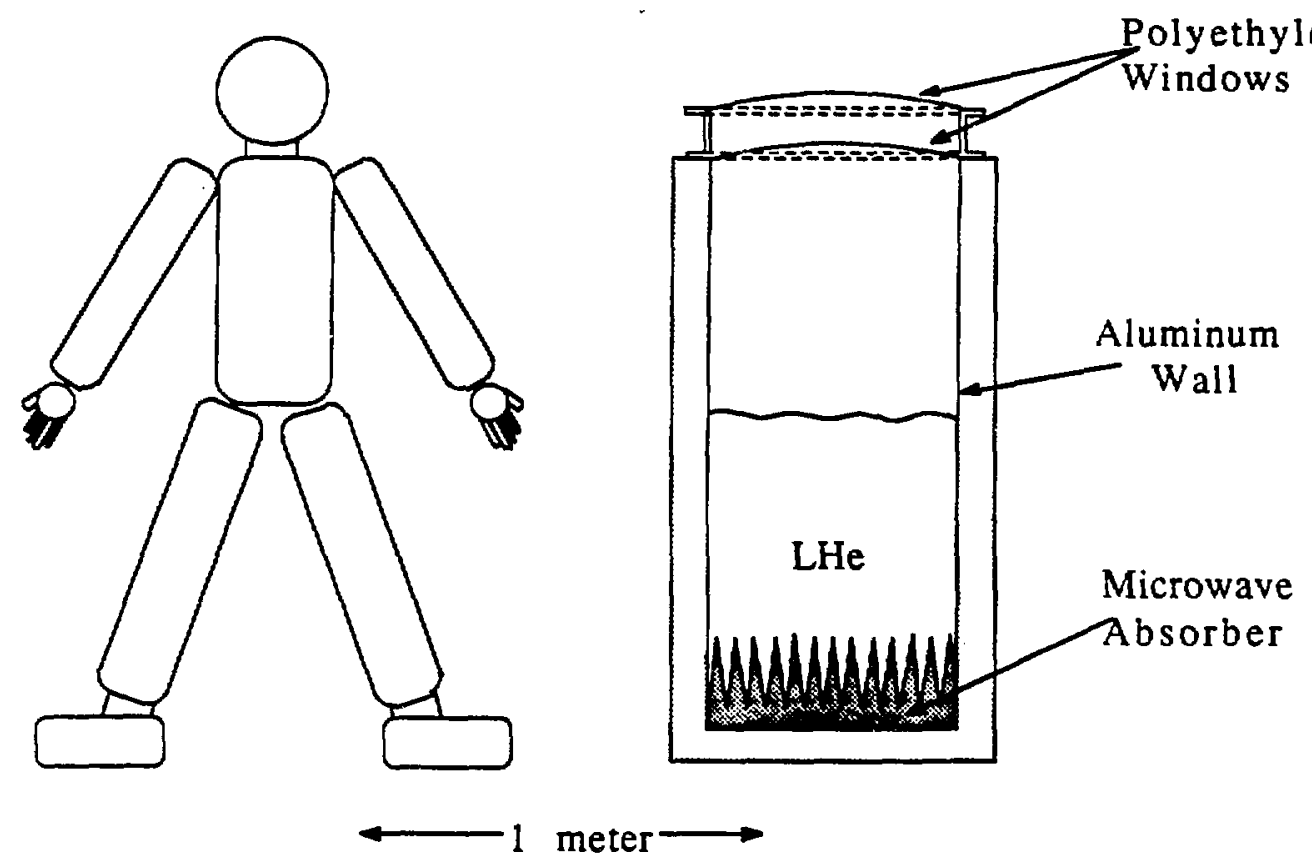

meter $\longrightarrow$

Figure 7. The low-temperature calibration target. 
signal was close to the signal received from the sky. By minimizing this temperature difference, we minimized the error induced by gain calibration error. As shown in Figure 7, the LHe target consists of a microwave absorber (Emerson-Cumings Eccosorb VHP-8) at the bottom of a cryogenic dewar. To minimize emission from the walls of the dewar, a false wall of aluminum-coated mylar runs from the Eccosorb to the opening at the top of the dewar. At the top of the dewar is a highly reflective movable shutter, which, when closed, greatly reduces heat leak from the surrounding environment to the cryogen. Above this shutter is another, short section of aluminum wall, with thin (20 micron) polyethylene windows above and below to prevent air from leaking into the cold load and condensing. This short section was continuously flushed with warm dry gas (boil-off gas from the cryogen was heated for the purpose) to keep condensation from forming on the windows.

The radiometer made an RF-tight seal with the top of the dewar by means of an interface plate shaped to match the surfaces of both the radiometer and the dewar. Alignment pins on the radiometer and corresponding slots in the plate ensured that the rectangular horn mated to the circular plate in a repeatable fashion. In normal operation the plate was fastened securely to the dewar, and the radiometer was held down on the plate by its own weight. When actually taking data, no one touched the radiometer or the dewar and the whole apparatus was motionless. 


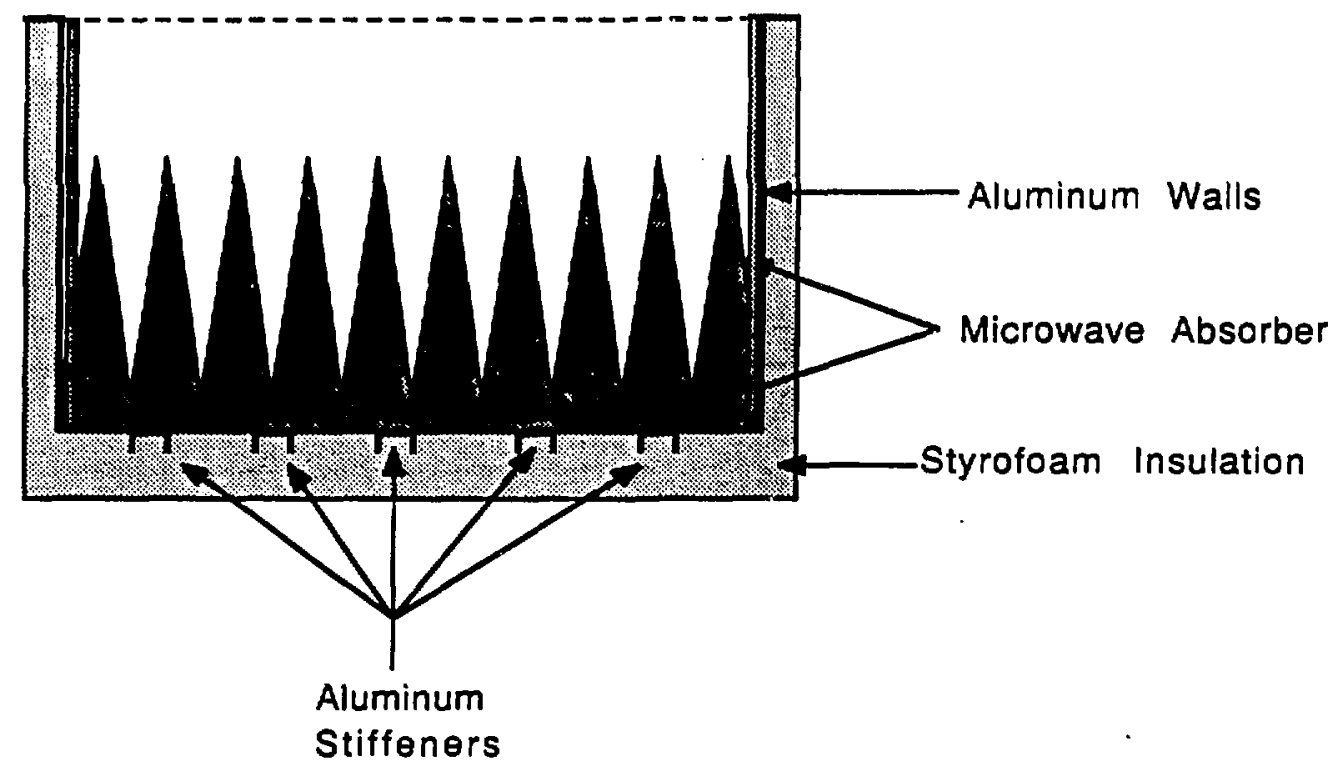
1 meter

Figure 8. The ambient-temperature calibration target. 
In order to determine the gain of the radiometer, we needed a known target with an antenna temperature much different from the LHe target. We therefore built an ambient-temperature target, and measured the gain of the radiometer by comparing the signal from the ambient-temperature target with the signal from the LHe target. The ambient target is a simplified, non-cryogenic version of the LHe target. It consists of a piece of Eccosorb at the end of an aluminum container (see Figure 8). Because we wished the target to remain unchanged under differing gravitational stresses (see Chapter V, Section iv), we stiffened the container with $4 \mathrm{~cm}$ aluminum angle, and lined the inside of the walls with absorber to minimize reflections. The walls were the same temperature as the target itself, so emission from the walls was not a problem. The outside of the target was covered with a $5 \mathrm{~cm}$ layer of styrofoam, to minimize temperature fluctuations. $A$ hole in the side of the container allowed us to insert a thermometer to measure the target's temperature. The bulk target temperature drifted less than $2 \mathrm{~K}$ per hour, and when not in use the target was covered with styrofoam insulation to minimize variation in the surface temperature. 
The $1.4 \mathrm{GHz}$ radiometer collects radiation with a broadband antenna, impedance matched to a coaxial cable. We built the antenna ourselves, and it is made from aluminum sheet, reinforced with $5 \mathrm{~cm}$ aluminum angle. In order to get low sidelobes in a reasonable size antenna, we used a corrugated design, described in Witebsky et al., (1987), reprinted as Appendix C. It is approximately 2 meters long, and detaches in the center (as shown in Figure 2) so that the radiometer can view the $\mathrm{LHe}$ calibrator.

Figure 9 shows a block diagram of the $1.4 \mathrm{GHz}$ radiometer. A microwave signal entering the antenna goes through a 3 stage GaAs FET amplifier, with $35 \mathrm{~dB}$ of gain. The input stage of this first amplifier is the dominant source of radiometer noise, because the signal is smallest here. Once the signal has been amplified, the signal to noise ratio for additional noise is high enough to make additional contributions to the $80 \mathrm{~K}$ system noise temperature negligible. Next there is a $2 \mathrm{~dB}$ attenuator to reduce reflections and to keep the final signal level within the optimum operating range of the detector diode. The signal then goes through a second amplifier (Miteq model $\mathrm{AM} 5 \mathrm{~A}$ 0420) with $50 \mathrm{~dB}$ gain, and then to a $1.0-1.6 \mathrm{GHz}$ bandpass filter. The bandpass filter eliminates any out-of-band RF interference which might otherwise cause a problem. A variety of radars and communications links operate at frequencies near $1.4 \mathrm{GHz}$ and, without the additional rejection of a bandpass filter, such signals 


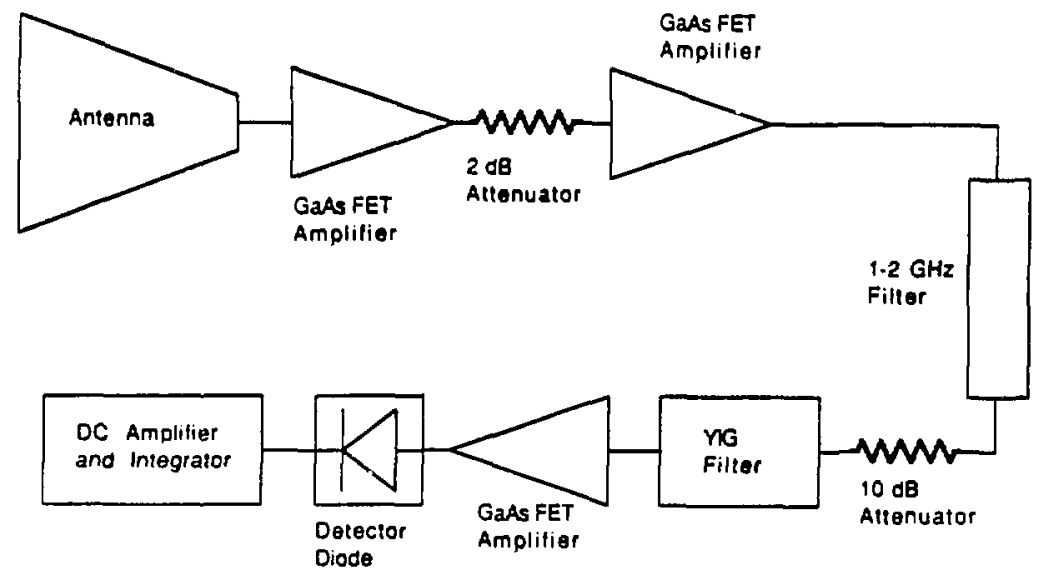

Figure 9. Schematic diagram of the $1.4 \mathrm{GHz}$ radiometer. 


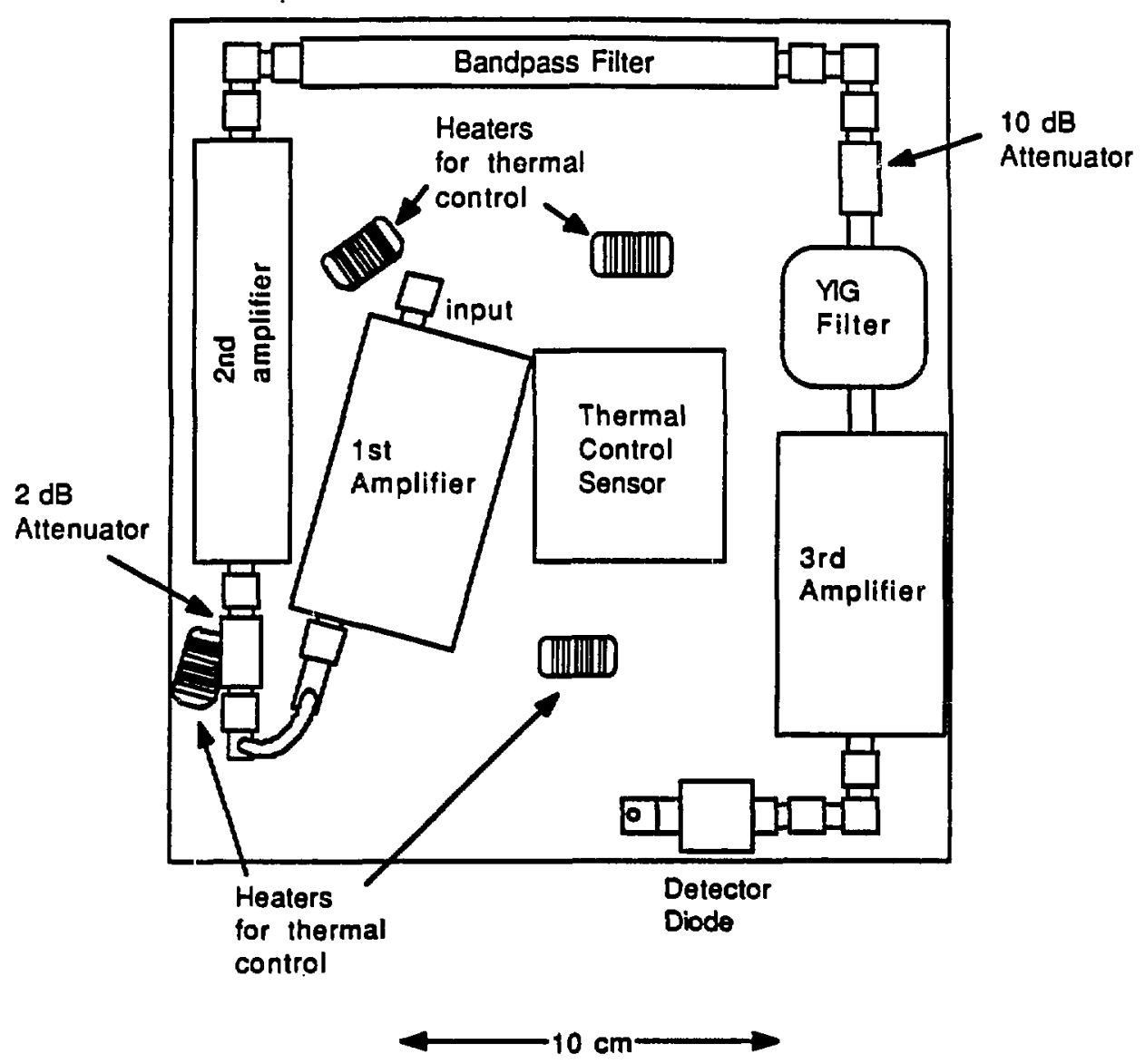

Figure 10. Physical layout of the $1.4 \mathrm{GHz}$ radiometer electronics. The backing plate is $1.3 \mathrm{~cm}$ thick aluminum to provide thermal contact. 
could give spurious, time-dependent results. RFI was much more of a problem in Berkeley than at the remote mountain observing site.

After the bandpass filter and another attenuator $(10 \mathrm{~dB})$ for more reflection padding, the signal goes through a Yttrium Iron Garnet (YIG) filter (YIG-TEK model 174-17). YIG is a ferritic material, and the heart of a YIG filter is a small YIG sphere suspended in a magnetic field. The field causes the aligned spins in the ferrite to precess, and the precession frequency is dependent on the magnitude of the magnetic field. By using this precession to produce a resonance affect, one can create a filter whose center frequency depends on the magnetic field. The field is then controlled with a small coil and a current source, and the result is an electronically tunable microwave filter. The YIG filter is magnetically shielded with mu-metal to reduce the effects of changes in the local magnetic field as the radiometer is moved about.

This particular YIG filter has a bandpass of $25 \mathrm{MHz}$, and an out-ofband rejection of $40 \mathrm{~dB}$. The current to the coils, and hence the center frequency of the filter, is controlled by an adjustable constant-current source. In normal operation, the current source electronically switches back and forth between two frquencies. It can be set with a toggle switch to use either of two pairs of frequencies, and the 4 frequency settings can be individually adjusted by opening the circuit box and changing one of 4 variable 
resistors.

After the 3rd amplifier (Watkins-Johnson model A43) boosts the signal again, it goes to a detector diode (Hewlett-Packard model 8473B). This nonlinear passive device is a square-law detector: Its output voltage is proportional to the square of the input voltage. Thus the d.c. level of the signal from the detector diode is proportional to the power in the RF signal going into it (and hence proportional to the RF power received by the antenna).

This d.c. voltage is then amplified a factor of 1000, from a few millivolts to a few volts, by a d.c. amplifier, which also integrates and averages the signal for 2 seconds (as clocked by the $100 \mathrm{~Hz}$ clock signal). While the integrator is accumulating its 2 -second average, the voltage displayed on the output is the average from the previous 2 -second period. From the integrator, the signal goes via coaxial cable to the $\mathrm{ADC}$ and tape recorder, as described in Chapter II.

The radiometer is thermally controlled to minimize gain drifts as a result of temperature-dependent variations in the gain of the amplifiers and the sensitivity of the detector diode. The YIG is also temperature-sensitive, but it has its own built-in thermal controller. The temperature regulating system for the $1.4 \mathrm{GHz}$ radiometer consists of 4 heaters and a thermistor-controlled circuit which adjusts the heater current to maintain a constant temperature. The heaters, the thermistor, and all the RF components are mounted on a 


\section{Radiometer Characteristics}

\begin{tabular}{|lrrr|}
\hline System Noise Temperature & & $80 \mathrm{~K}$ \\
\hline Sensitivity & $\begin{array}{c}0.017 \\
\mathrm{~K} / \sqrt{\mathrm{Hz}}\end{array}$ & $\begin{array}{c}\text { (theoretical) } \\
\text { (measured) }\end{array}$ \\
\hline Calibration Constant & $0.023 \mathrm{~K} / \sqrt{\mathrm{Hz}}$ & $46 \mathrm{~K} / \mathrm{V}$ \\
\hline Gain Stability & & 0.1 & $\% /$ minute \\
\hline Operating Frequency & 1.410 & $\mathrm{GHz}$ & (adjustable) \\
\hline Bandwidth & & & $0.025 \mathrm{GHz}$ \\
\hline
\end{tabular}

Table 1. Some important characteristics of the $1.4 \mathrm{GHz}$ radiometer. 
$1.3 \mathrm{~cm}$ aluminum plate for thermal stability. Contact between the plate and the antenna is limited to a short $(3 \mathrm{~cm})$ piece of stainless steel coaxial line, 2 stainless steel stand-offs, and the thin stainless steel RF shielding which surrounds the microwave components. Stainless steel was chosen because of its high ratio of strength to thermal conductivity. The antenna itself is not thermally regulated, but its large mass and aluminum construction serve to keep its temperature relatively constant.

Table 1 contains a summary of the radiometer characteristics and specifications. The system noise temperature is measured by comparing the signal when looking at a cold target (such as the LHe calibration target) with the signal from a warm target (such as the ambient-temperature target). The signal, $S_{\text {Target, seen when looking }}$ at a target is proportional to $T_{S Y S}+T_{\text {Target, }}$ so the system temperature is given by

$$
\frac{S_{\text {Cold }}}{S_{\text {Hot }}}=\frac{T_{\text {SYS }}+T_{\text {Cold }}}{T_{S Y S}+T_{\text {Hot }}}
$$

Hence

$$
T_{S Y S}=\frac{T_{\text {Hot }} S_{\text {Cold }}-T_{\text {Cold }} S_{\text {Hot }}}{S_{\text {Hot }}-S_{\text {Cold }}}
$$

The radiometer noise for an integration time $t$ is expected to be $\Delta \mathrm{T}=\frac{\mathrm{TSYS}}{\sqrt{\mathrm{Bt}}}$, where $\mathrm{B}$ is the bandwidth (Kraus, 1966). The measured value given in the table is based on the average variation of 2- 
second data samples taken with the radiometer at the remote site. The calibration constant and gain stability given in the table are based on comparison of the signal from the ambient-temperature target and the LHe reference target at the time of the actual CBR measurement, and are discussed in greater detail in Chapter $\mathrm{V}$, Section i. Gain variation is dominated by linear drifts (caused by temperature variation in the radiometer) on a time scale of several minutes. 
In late July of 1986 we went to the Nello Pace laboratory of the University of California's White Mountain Research Station on Mount Barcroft in the White Mountains, near Bishop, California. We arrived on July 25, 1986. The Nello Pace Laboratory is at an altitude of 3800 meters above sea level, and is crewed nearly year-round, although inaccessible to large vehicles during the winter months when snow closes the roads. In addition to the $1.4 \mathrm{GHz}$ radiometer, we brought radiometers designed to operate at frequencies of $3.7,10$, and 90 $\mathrm{GHz}$. Of the 4 radiometers, only the $90 \mathrm{GHz}$ radiometer had remained essentially unchanged since the last previous measurement, in August of 1984. Both the $1.4 \mathrm{GHz}$ radiometer and the $3.7 \mathrm{GHz}$ radiometer were newly constructed, and there were still minor additions to be made to the $1.4 \mathrm{GHz}$ radiometer (such as the magnetic shielding on the YIG filter and the last of the thermal insulation).

The research team consisted of Jon Aymon, Marc Bensadoun, Marco Bersanelli, Giovanni De Amici, John Gibson, Al Kogut, Steve Levin, Larry Levin, George Smoot, Carol Stanton, and Chris Witebsky, from U.C. Berkeley's Space Sciences Laboratory and Lawrence Berkeley Laboratory.

While readying the support equipment, such as the cold load and the computers, we finished construction and testing of the $1.4 \mathrm{GHz}$ radiometer. Using the radiometer with the $R F$ output sent to a spectrum analyzer, we determined that man-made sources of interference (RFI) were absent at the $10 \mathrm{mK}$ level. Based on 


\section{Return Loss from LHe Dewar}

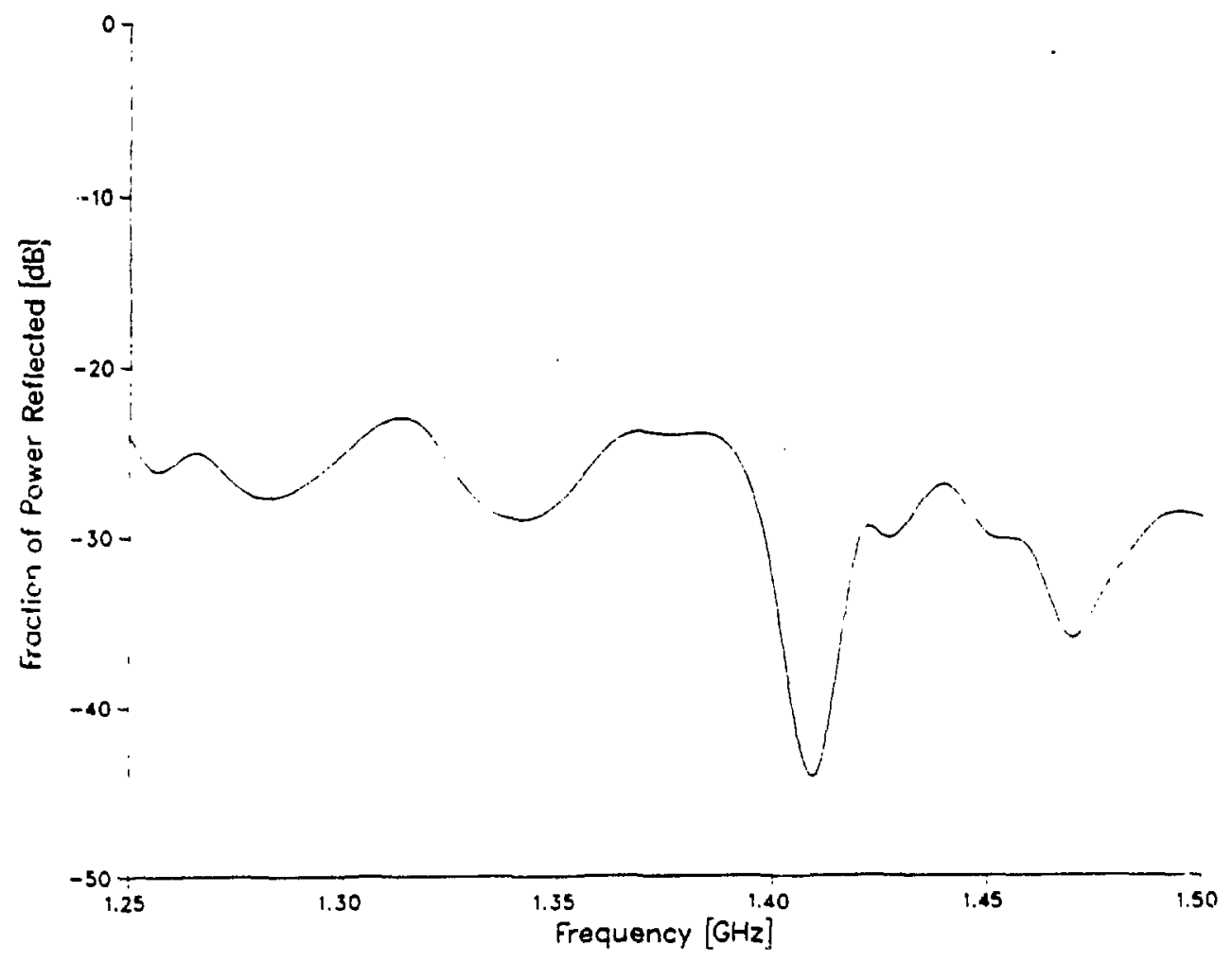

Figure 11. Return loss from the LHe dewar as seen through the 1.4 $\mathrm{GHz}$ antenna. 
measurements of the dewar reflection (see figure 11) and the antenna bandwidth, we chose operating frequencies of $1.410 \mathrm{GHz}$ and $1.465 \mathrm{GHz}$, and frequencies of 1.300 and $1.465 \mathrm{GHz}$ for galactic scan measurements.

On August 1, 1986, while trying to improve the impedance match between the radiometer input and the coax line leading from the antenna, we accidently destroyed the input stage to the 1st amplifier in the $1.4 \mathrm{GHz}$ radiometer. We arranged by radiotelephone to borrow a replacement from Dave Williams at the University of California's Radio Astronomy Lab on the Berkeley campus, and it was sent to us at Barcroft. In the meantime, the weather forecast, while acceptable for the next few days, predicted rain in about a week. A decision was made to begin measurements before the rains made it impossible. The cold load was filled with liquid nitrogen for final tests, and liquid helium was sent up the mountain, giving us a firm deadline (LHe is lost from the dewars at a rate of about $1 \%$ per day.)

The replacement amplifier arrived on August 3, and was immediately installed in the radiometer, necessitating changes in the component layout to accommodate its size and shape. Because of the changes, we repeated the vertical flip tests (discussed in Chapter $V$, Section iv) and the gain stability tests, as well as measuring the new gain, system temperature, and input impedance.

On August 7 , we began practice runs with liquid nitrogen in the 
dewar. Preliminary results showed a significant difference between the calibration target temperature at $1.465 \mathrm{GHz}$ and at $1.410 \mathrm{GHz}$. (Later analysis confirmed that this was caused by reflection problems, discussed in Chapter V, Section ii.) On August 8, 1986 we began measurements of the CBR. We made measurements with the LHe calibrator from 2:37 Universal Time (UT) to $3: 32$ UT, from $6: 43$ to 7:38 UT (while the galactic plane was nearly overhead) and from 10:35 until the LHe ran out at 11:30 UT. In the first few minutes over the LHe target, we realized that LHe loss would be unacceptably high if the radiometer were to view the LHe target with a $45 \%$ duty cycle, as originally planned. We therefore changed to the following cycle, used for all subsequent runs over the dewar:

1) The radiometer viewed the sky for 64 seconds.

2) The radiometer viewed the LHe target for 64 seconds

3) The radiometer viewed the sky again for 64 seconds 64 seconds

4) The radiometer viewed the ambient-temperature target for

This cycle was repeated throughout the run. When not being viewed, the LHe dewar was covered with a highly reflective shutter to minimize cryogen loss. Moving the radiometer to view a different target always took less than 16 seconds, and normally took less than 5. In the analysis, data from the 6 seconds immediately after the radiometer was moved were discarded, as were any anomalous records. A total of 8 out of 172 records were thrown out. 
The original plan was to use the $1.4 \mathrm{GHz}$ radiometer for galactic scans when it was not over the dewar, but because manpower was needed on the other radiometers, and because of concerns (later found to be groundless) that operation of the noise source used to calibrate such scans would interfere with the $3.7 \mathrm{GHz}$ radiometer, we only did galactic scans from $7: 46$ to $10: 02$ and from 11:54 to 13:05. On August 9, problems with the LHe target resulted in an wbnormally high rate of LHe loss, and the available measurement time was reduced. Measurements were made from $6: 39$ to $7: 29$ UT and from 10:08 to 11:02 UT. A galactic scan was done from 7:37 to 9:53 UT.

On August 10, 1986 we ran various tests of the radiometer, and performed a galactic scan from 6:08 UT until sunrise (see Chapter V, Section iii for details). Throughout the galactic scan, there were lightning flashes from a rainstorm on the eastern horizon, and the weather at the site, while not actually raining, was poor. We removed the quarter-wave traps from the antenna, and alternately returned and removed one of the traps, to look for changes in signal as an estimate of sidelobe pickup. Results of these and related tests are discussed in Chapter V.

As well as the actual measurement of the CBR and the Galactic signal, we did a variety of tests to ensure that the radiometer was operating properly and was well understood. The major tests of radiometer performance were tests for stability, gravitationally induced changes, magnetic sensitivity, temperature sensitivity, sensitivity to 
microphonics, and gain linearity.

On 8/6/86 at 6:09 UT, we did a vertical flip test with the radiometer, turning it upside down and back repeatedly to test for effects from gravitational stress and magnetic sensitivity. Details are discussed in Chapter V, Section iv. The actual data-taking sequence over the LHe dewar included measurement of the signal from an ambient target as well as from the sky and the LHe target. The best tests of gain stability and temperature sensitivity were these gain and system temperature measurements performed as part of the measurement sequence. An increased variability in the radiometer output during the CBR measurements and the flip test does indicate some sensitivity to microphonics, but the resulting fluctuations in the measurement of the sky/LHe antenna temperature difference were not a major contributor to the error budget (See Chapter V, Section i). Gain linearity was verified on $7 / 15 / 86$ by inserting known attenuators in the amplification chain of the radiometer, and saturation effects were absent at the $3 \%$ level. 
As discussed in Chapter II, the basic concept of this experiment is to measure the difference between the signal from the sky and the LHe target, and calculate the CBR signal by determining and subtracting all other contributions. In this chapter I will describe the analysis used to determine those additional contributions and their attendant errors.

The signal difference between the sky and the LHe target is

$$
\begin{aligned}
G\left(S_{\text {zenith }}-S_{\text {load }}\right)=T_{\text {zenith }}-T_{A, \text { load }} \\
=T_{A, \text { CBR }}+T_{\text {galaxy }}+T_{\text {atmosphere }}+T_{\text {ground }}+\Delta T_{\text {offset }}-T_{A, \text { load }}
\end{aligned}
$$

where $G$ is the radiometer's calibration constant; $S_{z e n i t h}$ is the radiometer output when viewing the zenith; $S_{\text {load }}$ is the radiometer output when viewing the cold-load LHe calibrator; $\boldsymbol{T}_{\text {zenith }}$ is the antenna temperature of the sky as seen by the radiometer looking vertically; $\mathrm{T}_{\mathrm{A}, \text { load }}$ is the antenna temperature of the LHe calibrator as seen by the $1.4 \mathrm{GHz}$ radiometer; $\mathrm{T}_{\mathrm{A}, \mathrm{CBR}}$ is the quantity we're trying to measure, the antenna temperature of the cosmic background radiation; $T_{\text {galaxy }}$ is the antenna temperature of the galaxy during this observation; $T_{a t m o s p h e r}$ is the antenna temperature of the atmosphere as seen by the $1.4 \mathrm{GHz}$ radiometer observing the zenith; $T_{\text {ground }}$ is the contribution from the Earth, picked up by diffraction in the antenna sidelobes; and $\Delta \mathrm{T}_{\text {offset }}$ is any change in the radiometer output due to gravitational stresses as the radiometer is turned over to view the LHe target. Solving for $T_{A, C B R}$ : 


$$
\begin{aligned}
T_{A, C B R}= & G\left(S_{\text {zenith }}-S_{\text {load }}\right) \\
& -T_{\text {galaxy }}-T_{\text {atmosphere }}-T_{\text {ground }}-\Delta T_{\text {offset }}+T_{A, \text { load }} .
\end{aligned}
$$

In order to determine $T_{A, C B R}$, we analyze each of the terms in Equation 8 separately. 


\section{$G\left(S_{\text {zenith }}-S_{\text {load }}\right)$}

During the 3 runs over the LHe dewar, 180 measurements were taken of $\left(S_{\text {zenith }}-S_{\text {load }}\right)$. 8 measurements were rejected, as having anomalously high signal values caused by changing targets too slowly (See Table 2). Using the concurrently measured values of the calibration constant, the measured value of $G\left(S_{z e n i t h}-S_{l o a d}\right)$ is $-0.06 \pm 0.03$ Kelvin, where the error is based solely on the statistical fluctuations in the measurement, and assumes a Gaussian parent distribution.

The gain does not vary with a Gaussian distribution, but in fact shows slow drifts (probably caused by temperature variation). Figure 12 is a plot of the measured gain as a function of time for 3 runs over the LHe target. The maximum spread between any 2 gain measurements was less than $5 \%$ and the limit on gain saturation effects is less than $3 \%$, so even the most conservative assumptions predict that the error induced by gain variation is less than $8 \%(0.06$ $\mathrm{K})=0.005 \mathrm{~K}$. The final result for this term is thus

$$
G\left(S_{\text {zenith }}-S_{\text {load }}\right)=-0.06 \pm 0.03 \mathrm{~K}
$$

The corresponding result at $1.465 \mathrm{GHz}$ is anomalously low $(-3.02 \pm 0.03 \mathrm{~K})$, and will be discussed further in the next section. 


\section{G(Szenith - Sload $)$}

\begin{tabular}{|c|c|c|c|c|c|}
\hline $\begin{array}{l}\text { TIME } \\
\text { (UT) }\end{array}$ & $\begin{array}{l}\text { FREQ. } \\
(\mathrm{GHz})\end{array}$ & $\begin{array}{l}\text { \# OF PTS. } \\
\text { (removed) }\end{array}$ & $\begin{array}{l}\text { AVE. } \\
(\mathrm{K})\end{array}$ & $\begin{array}{l}\text { RMS } \\
\text { (K) }\end{array}$ & $\begin{array}{c}\sigma \\
(\mathrm{K})\end{array}$ \\
\hline \multicolumn{6}{|l|}{$8 / 8 / 86$} \\
\hline $2: 40-$ & 1.410 & 57 (1) & -0.0271 & 0.4277 & 0.0567 \\
\hline $3: 30$ & 1.465 & $58(0)$ & -3.384 & 0.2643 & 0.0347 \\
\hline $10: 40-$ & 1.410 & 60 (1) & +0.0052 & 0.2709 & 0.0350 \\
\hline $11: 30$ & 1.465 & $61(0)$ & -2.953 & 0.3131 & 0.0401 \\
\hline \multicolumn{6}{|l|}{$8 / 9 / 86$} \\
\hline $10: 00-$ & 1.410 & $63(6)$ & -0.1599 & 0.5156 & 0.0650 \\
\hline $11: 00$ & 1.465 & $68(0)$ & -2.712 & 0.6185 & 0.0750 \\
\hline
\end{tabular}

Table 2. Measurements of sky temperature minus LHe temperature. Each data point represents the difference between two 16-second records. 
Gain of $1.41 \mathrm{GHz}$ Radiometer on 8/8/86

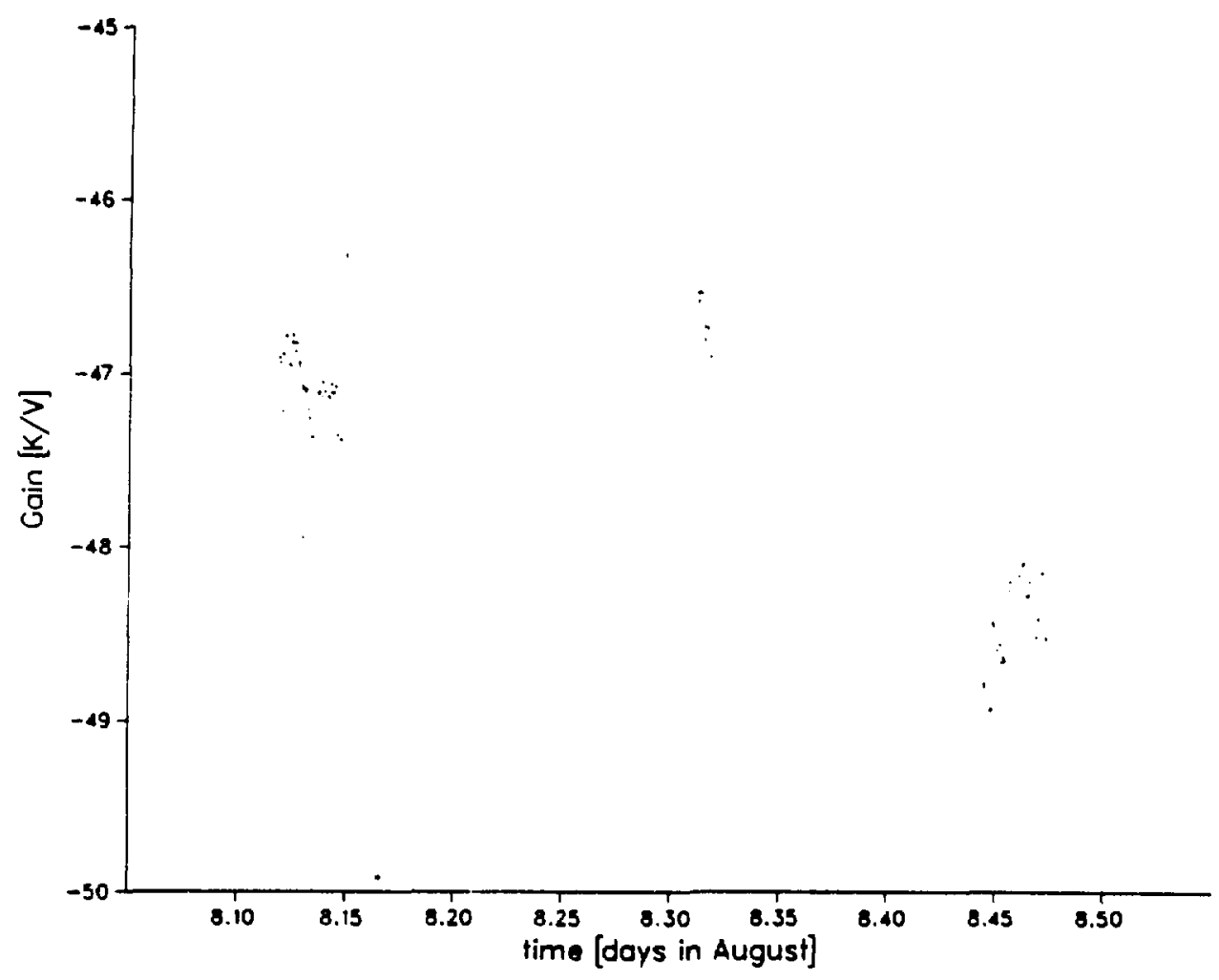

Figure 12. Gain as function of time for LHe measurements made on $8 / 8 / 86$. 


\section{Absolute Reference Load}

$T_{A, l o a d}$, the antenna temperature of the cold load, depends both on the power emitted by the cold load, and on the power reflected from the cold load. The measured atmospheric pressure during measurements was $489 \pm 2 \mathrm{~mm}$ of mercury, so the boiling temperature of helium was $3.782 \pm 0.004 \mathrm{~K}$ (Donnelly, 1967). Thus, the Eccosorb had an antenna temperature of $3.748 \pm 0.004 \mathrm{~K}$. The power emitted by the polyethylene windows and by the walls is small, $0.003 \pm 0.002$ and $0.019 \pm 0.019 \mathrm{~K}$, respectively.

The cold load reflects power as well as emitting it, and the radiometer, as does any object above absolute zero, emits radiation. Because the LHe target is not a perfect absorber, a small fraction of the power emitted by the radiometer is reflected from the cold load back into the antenna, and contributes to the antenna temperature of the cold load. The power broadcast by the radiometer should be equivalent to the system temperature, or roughly $100 \mathrm{~K}$, and we measured the power reflection coefficient of the dewar as viewed through the antenna, finding it to be $-34 \mathrm{~dB}$, so the contribution would, on the surface, appear to be only about $0.030 \mathrm{~K}$. Unfortunately, this is not the case. Because the bandwidth of the radiometer is only $25 \mathrm{MHiz}$, its coherence length is approximately 12 meters. This is longer than the length of the dewar, so reflections from the LHe target can interfere coherently with reflections from inside the radiometer itself (see Figure 13). 


$$
\begin{aligned}
E & =r_{1} E_{0} e^{i \phi}+r_{2} E_{0} e^{i \theta} \quad\left(r_{1}<1\right) \\
I & =|E|^{2} \\
|E|^{2} & =E_{0}{ }^{2} r^{2}+E_{0}{ }^{2} r_{2}^{2}+2 E_{0}{ }^{2} r_{1} r_{2} \cos (\theta-\phi)
\end{aligned}
$$

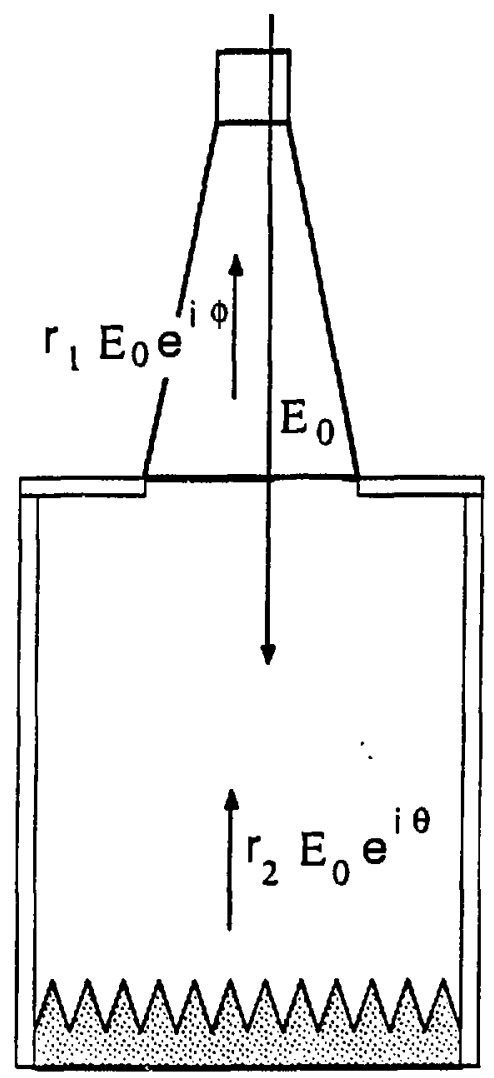

Figure 13. Coherent reflection. Reflections from within the radiometer can interfere coherently with reflections from the target. 
If the radiometer broadcasts power of intensity $E_{0}{ }^{2}$, then the power reflected from the LHe target is

$$
\begin{aligned}
& E^{2}=E_{0}^{2} r_{1}^{2}+E_{0}^{2}\left(1-r_{1}^{2}\right) r_{2}^{2}+2 E_{0}^{2}\left(1-r_{1}^{2}\right) r_{1} r_{2} \cos (\Delta \Phi) \\
& E^{2}=E_{0}^{2} r_{1}^{2}+E_{0}^{2} r_{2}^{2}+2 E_{0}^{2} r_{1} r_{2} \cos (\Delta \Phi)
\end{aligned}
$$

where $r_{1}$ is the magnitude of the amplitude reflection coefficient for reflections inside the radiometer, $r_{2}$ is the magnitude of the amplitude reflection coefficient for reflections from the target, $\Delta \Phi$ is the net phase difference between the two reflected signals when they recombine, and $I$ have assumed $r_{1}<<1$.

The first term in Equation 9 does not affect the measurement, because it remains unchanged when we compare the signal from the sky with the signal from the LHe target. The second term in Equation 9, which represents the power reflected from the target when coherence effects are neglected, is small, as we saw above. This leaves the final, phase-dependent term. Not having anticipated the magnitude of the reflection effect, we were not prepared to measure the reflection phase difference during actual measurements at the remote site on Barcroft mountain. There are, however, a number of ways to determine the magnitude of the effect (if not its sign) after the fact.

Before and after taking data at the remote site, we measured the power reflection coefficient of the LHe target, the radiometer 


\begin{tabular}{|c|c|c|c|}
\hline Frequency & Target & $\begin{array}{l}\text { Return } \\
1 \mathrm{MHz} \text { BW }\end{array}$ & $\begin{array}{l}\text { Loss } \\
25 \mathrm{MHz} \text { BW }\end{array}$ \\
\hline $1.410 \mathrm{GHz}$ & cold-load calibrator & $44 \mathrm{~dB}$ & $34 \mathrm{~dB}$ \\
\hline $1.410 \mathrm{GHz}$ & $\begin{array}{c}\text { ambient-temperature } \\
\text { calibrator }\end{array}$ & $27 \mathrm{~dB}$ & $27 \mathrm{~dB}$ \\
\hline $1.410 \mathrm{GHz}$ & antenna (sky) & $30 \mathrm{~dB}$ & $29 \mathrm{~dB}$ \\
\hline $1.410 \mathrm{GHz}$ & antenna extension & $39 \mathrm{~dB}$ & $38 \mathrm{~dB}$ \\
\hline $1.410 \mathrm{GHz}$ & $\begin{array}{l}\text { input to } 1 \text { st amplifier } \\
\text { (sensitive to load) }\end{array}$ & $(22 \mathrm{~dB})$ & $(22 \mathrm{~dB})$ \\
\hline $1.465 \mathrm{GHz}$ & old-load calibrator & $35 \mathrm{~dB}$ & $32 \mathrm{~dB}$ \\
\hline $1.465 \mathrm{GHz}$ & $\begin{array}{c}\text { ambient-temperature } \\
\text { calibrator }\end{array}$ & $34 \mathrm{~dB}$ & $33 \mathrm{~dB}$ \\
\hline $1.465 \mathrm{GHz}$ & antenna alone & $34 \mathrm{~dB}$ & $33 \mathrm{~dB}$ \\
\hline $1.465 \mathrm{GHz}$ & antenna with extension & $43 \mathrm{~dB}$ & $42 \mathrm{~dB}$ \\
\hline $1.465 \mathrm{GHz}$ & $\begin{array}{l}\text { input to } 1 \text { st amplifier } \\
\text { (sensitive to load) }\end{array}$ & $(24 \mathrm{~dB})$ & $(24 \mathrm{~dB})$ \\
\hline
\end{tabular}

Table 3. Power reflection coefficients from various targets, as measured with the directional coupler and spectrum analyzer. Return loss is given both for the $1 \mathrm{MHz}$ resolution of the measurement, and averaged over $25 \mathrm{MHz}$, to approximate the radiometer's bandwidth. 


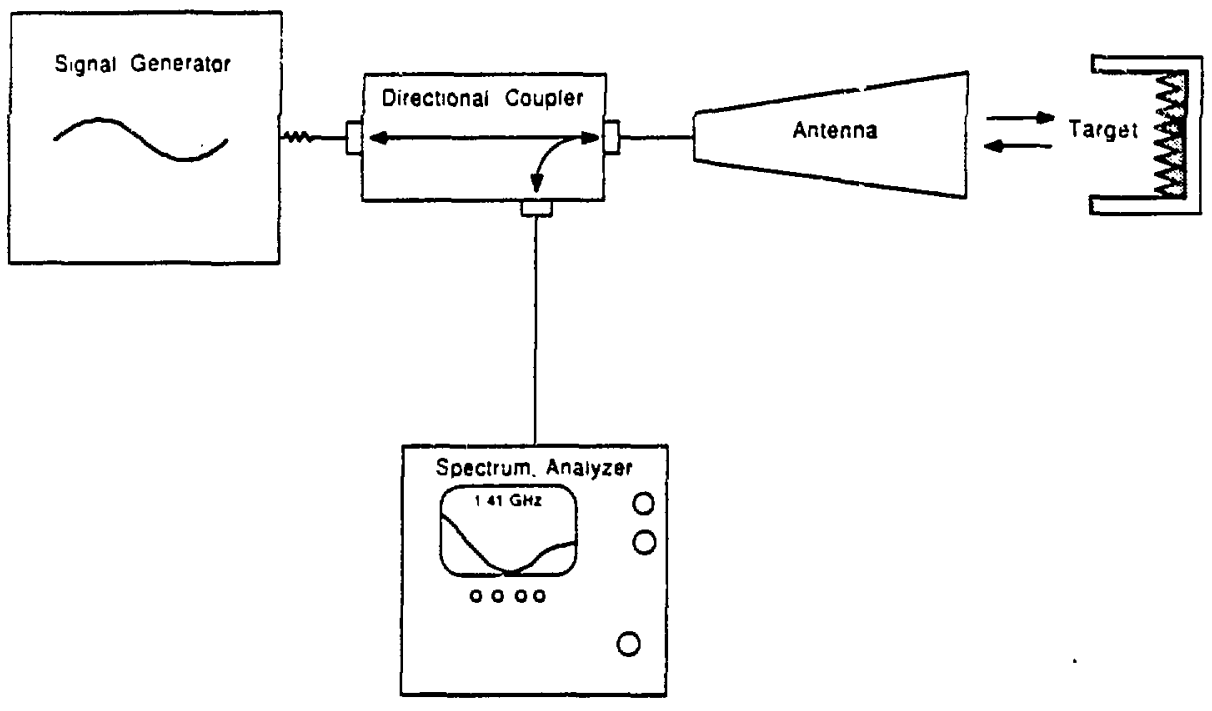

Figure 14. Apparatus used for measuring return loss. 
antenna, the ambient target, and the interface plate to the cold-load (See Table 3). The setup used to make these measurements is shown in Figure 14. We sent a large signal through a directional coupler to the antenna (and hence on to the target) and used a spectrum analyzer to look at the reflected signal through the directional port. The signal received at the spectrum analyzer was therefore proportional to the power reflected by the antenna/target combination. By replacing the antenna with a piece of shorted coaxial line, we saw the signal for $100 \%$ reflection. Hence the ratio (logarithmic difference) of the two signals gave us the power reflection coefficient of the antenna/target combination.

To measure the reflection from the antenna itself we used the sky as a target. We also measured the input impedance of the radiometer by sending a signal into the input of the first amplifier in the same fashion. Because the input impedance depends partly on the load seen by the radiometer, this measurement must be viewed somewhat skeptically. In principle, however, the input impedance, combined with the reflection measurements of the LHe target and the antenna, should enable us to determine the amplitude (but not the phase) of the last term in Equation 9. In practice, we decided to investigate the reflection effects more thoroughly.

First, to verify that we understood the effect, we constructed a test apparatus (see Figure 15) to examine the variation of radiometer 


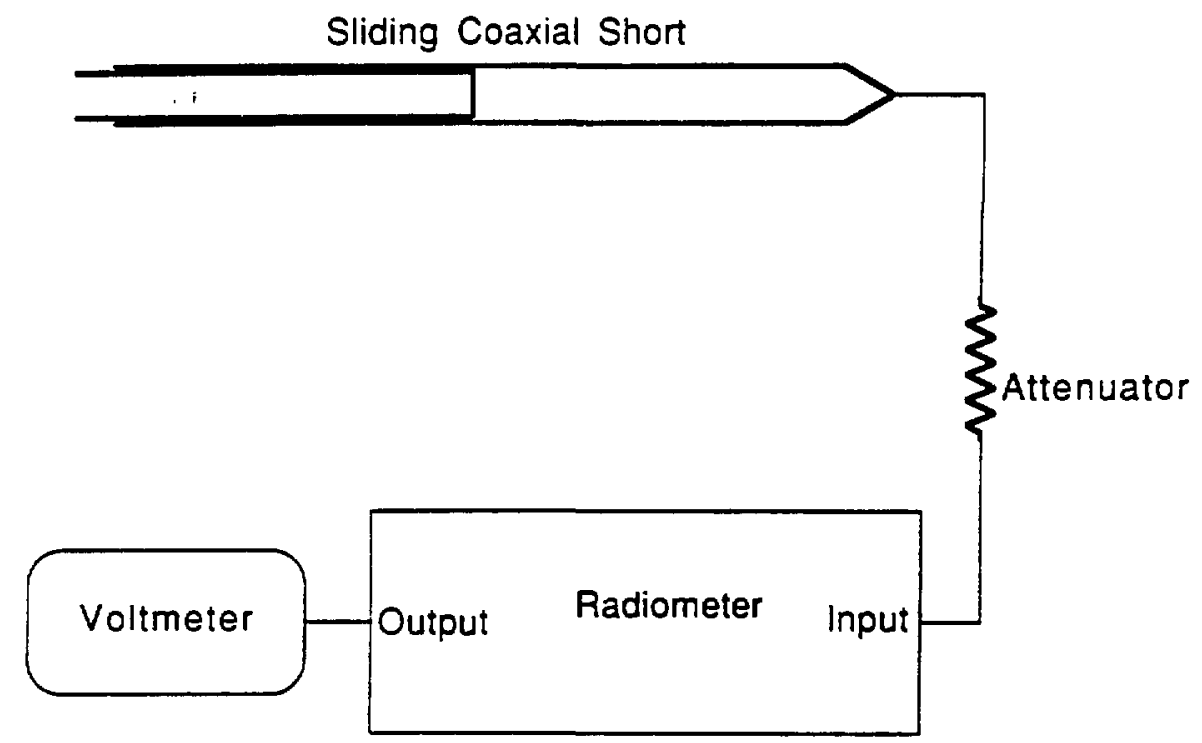

Figure 15. Schematic of sliding short apparatus used to measure the radiometer's response to reflections. 
Phase Variation of Reflection Response

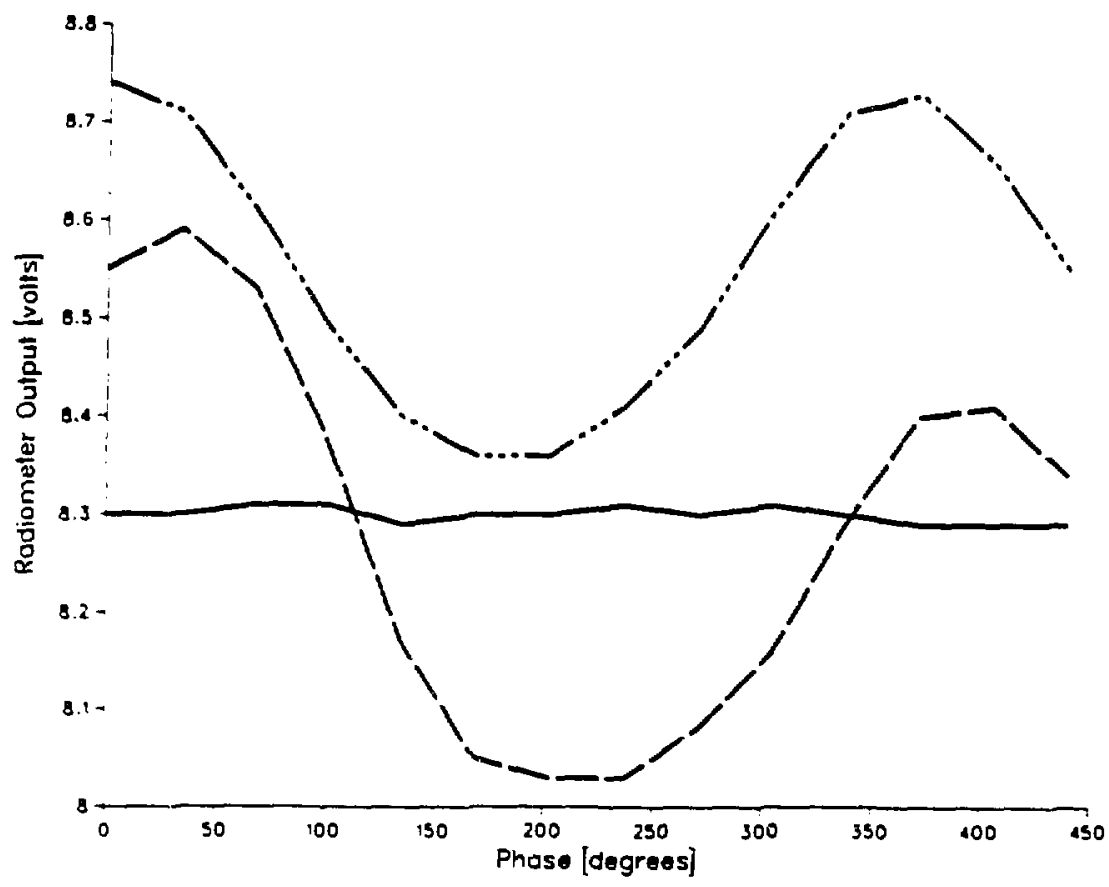

Legend

16 de altenuatc

$5 \mathrm{~dB}$ attenuator

to ab oftenuate

Figure 16. Radiometer signal as a function of phase for reflections seen through various attenuators. The equivalent return loss (in $\mathrm{dB}$ ) is iwice the value of the attenuator used. 
output as we changed the phase of the reflected signal. By causing the radiometer to view a shorted piece of coaxial line through an attenuator, we created a known partial reflector, whose reflection coefficient could be changed simply by switching attenuators. We varied the phase by moving the sliding short up and down the coaxial line. In this fashion, we mapped the radiometer response to various values of the phase and reflection coefficient. Sample plots of radiometer output versus phase are shown in Figure 16. As expected, the output of the radiometer varies sinusoidally with the phase of the reflected signal.

Operating the radiometer and sliding short system at both $1.410 \mathrm{GHz}$ and $1.465 \mathrm{GHz}$, we quickly found that the radiometer was several times more sensitive to reflections at $1.465 \mathrm{GHz}$ than at $1.410 \mathrm{GHz}$, in keeping with the anomalously low results for $G\left(S_{z e n i t h}-S_{\text {load }}\right)$ at $1.465 \mathrm{GHz}$. Presumably this is due to frequency-dependence in the radiometer's response to reflections. In any event, we have rejected the data taken at $1.465 \mathrm{GHz}$, and concentrated on measurements made at $1.410 \mathrm{GHz}$. Table 4 shows the amplitude of the sinusoidal variation with phase in the radiometer output for various values of reflection coefficient and frequency.

Because of weather conditions in the White Mountains, the LHe target itself is inaccessible until spring, but we built a mock-up of the LHe dewar, and examined the radiometer response to phase 


$\begin{array}{cccc}\begin{array}{c}\text { Frequency } \\ \text { (GHz) }\end{array} & \text { Attenuator } & \begin{array}{c}\text { Equivalent } \\ \text { Return Loss }\end{array} & \begin{array}{r}\text { Peak-Peak } \\ \text { Amplitude }\end{array} \\ 1.410 & 16 \mathrm{~dB} & 32 \mathrm{~dB} & <0.9 \mathrm{~K} \\ 1.410 & 10 \mathrm{~dB} & 20 \mathrm{~dB} & 16 \mathrm{~K} \\ 1.410 & 6 \mathrm{~dB} & 16 \mathrm{~dB} & 22 \mathrm{~K} \\ 1.410 & 0 \mathrm{~dB} & 0 \mathrm{~dB} & 33 \mathrm{~K} \\ 1.465 & 16 \mathrm{~dB} & 32 \mathrm{~dB} & 6 \mathrm{~K} \\ 1.465 & 10 \mathrm{~dB} & 20 \mathrm{~dB} & 9 \mathrm{~K} \\ 1.465 & 6 \mathrm{~dB} & 12 \mathrm{~dB} & 28 \mathrm{~K}\end{array}$

Table 4. Response of radiometer to phase variation of reflections, using the sliding short apparatus described in the text. 


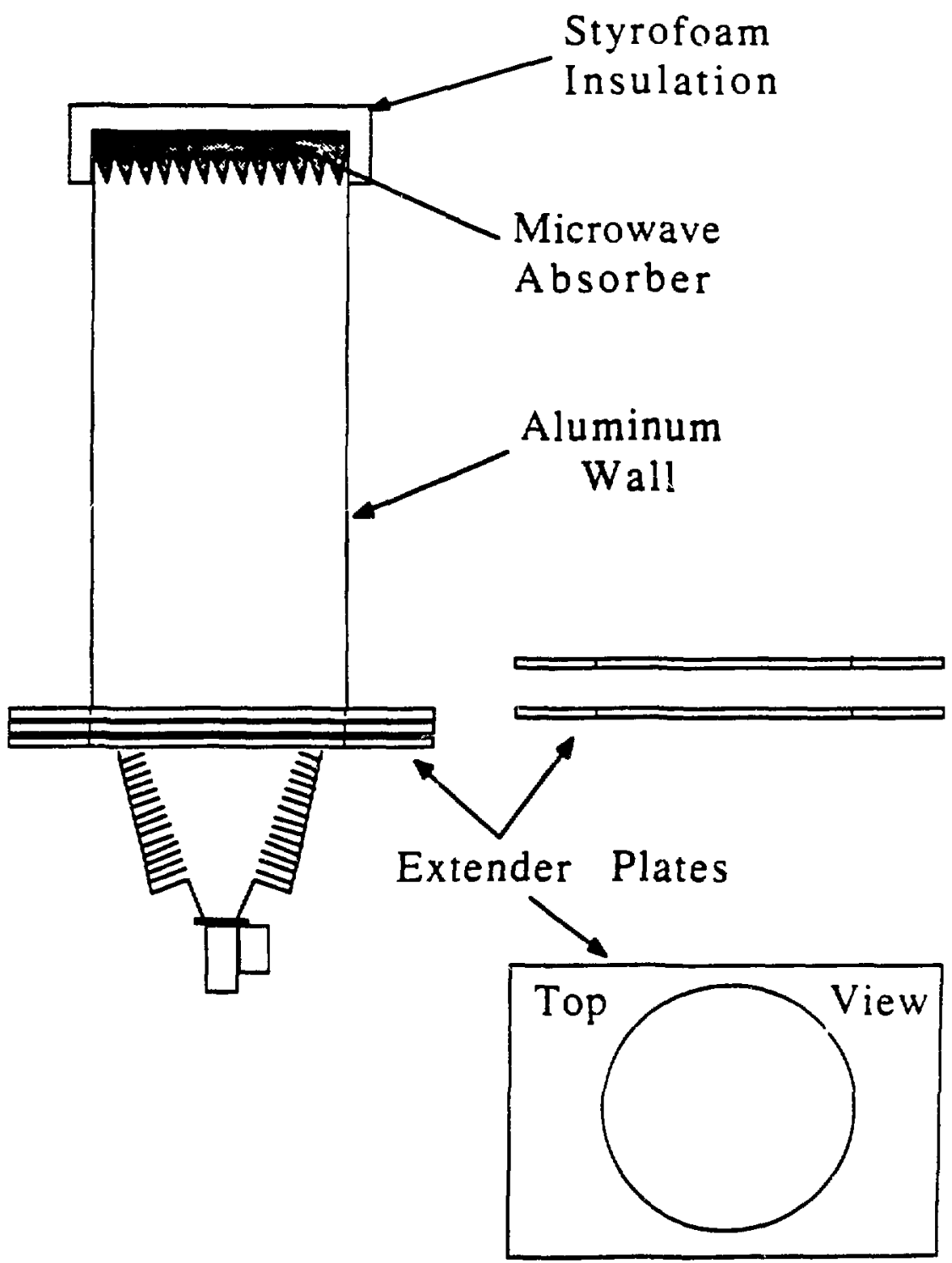

Figure 17. Mock-up used to simulate the LHe dewar for reflection purposes. 
Varying Length of Dewar Mockup: $1.410 \mathrm{GHz}$

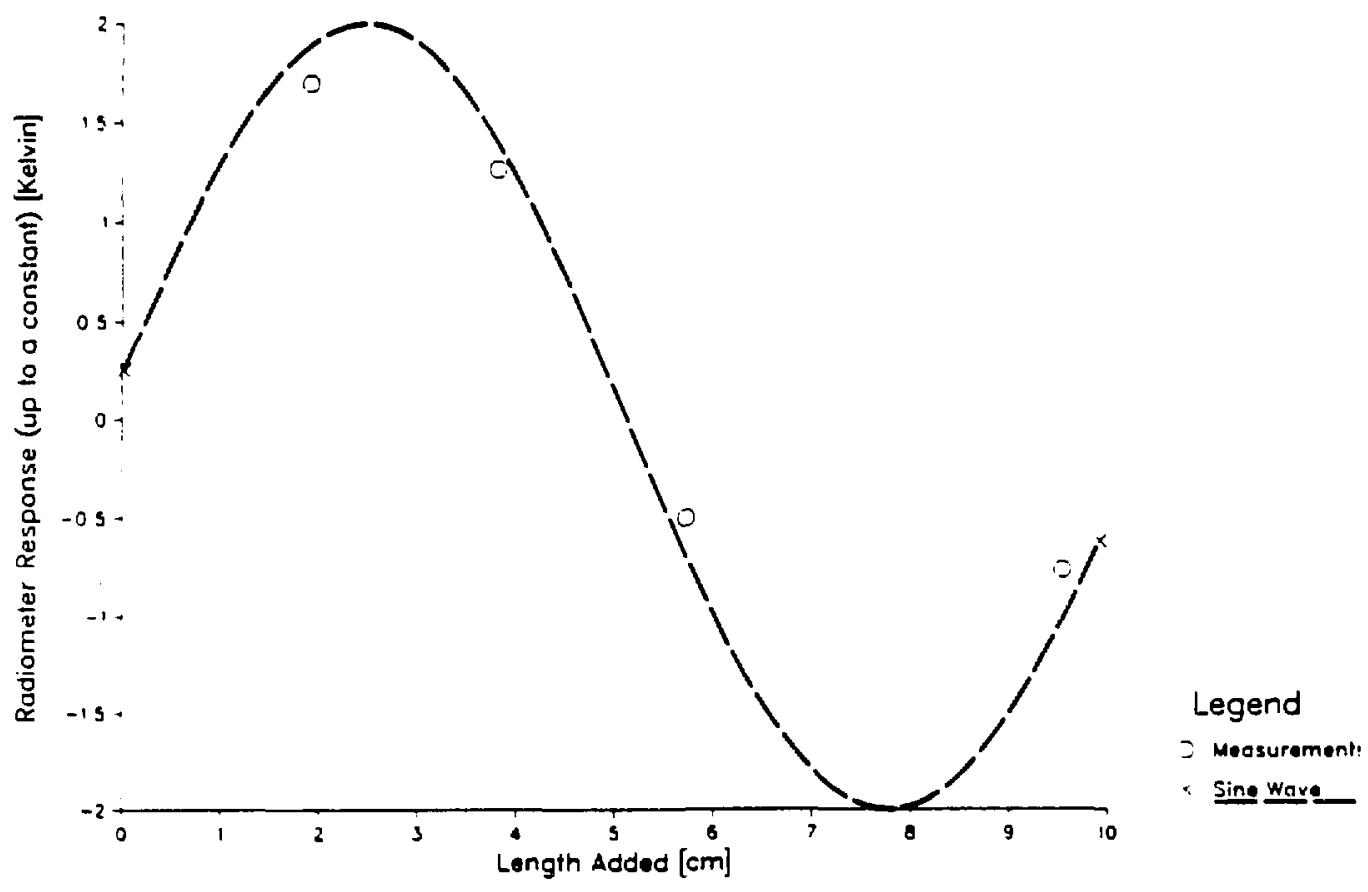

Figure 18. Radiometer output when viewing simulated dewar, as a function of phase. The $2 \mathrm{~K}$ sine wave shown has phase adjusted to fit the data points. 
variation in Berkeley by effectively varying the length of the dewar wall (see Figure 17). We were able to see the sinusoidal variation of radiometer output with reflection phase, but return loss measurements with the directional coupler showed that the mock-up was not sufficiently like the real LHe dewar to justify using the phase of the reflections measured on the mock-up to estimate the effect from the real thing. As a result, the phase of the reflection term is unknown, and its amplitude is determined by measurements of the mock-up, as well as by the sliding-short measurements mentioned above, along with the return loss measurements performed on the target in situ.

Figure 16 shows the results of the sliding short tests with various attenuators. The equivalent return loss is just twice the attenuator value. The measured return loss of the LHe target at $1.410 \mathrm{GHz}$ was $34 \mathrm{~dB}$, and the reflection response of the radiometer at $1.410 \mathrm{GHz}$, as measured with sliding short, was less than $0.5 \mathrm{~K}$ for a return loss of $32 \pm 2 \mathrm{~dB}$.

Measurements of the phase variation of reflections from the mock dewar show a sine wave with amplitude $2.0 \mathrm{~K}$ (see Figure 18). The reflection effect, however, involves reflection of the signal emitted by the target, as well as of the signal emitted by the radiometer. Because the radiation emitted by the radiometer is incoherent with the radiation emitted by the target, we can treat the two reflection 
problems separately, and simply add the power which arrives at the radiometer input after being emitted by the target to the power which arrives there after being emitted by the radiometer and reflected.

If the reflection is symmetric with respect to direction, as is the case for any system with no non-reciprocal elements (such as ferrites or active components), the same fraction of power is reflected back to the target as was reflected back to the radiometer (Ramo et al., 1965). Thus, if the fractional power reflected back to the receiver is $\frac{\left|E_{R r}\right|^{2}}{\left|E_{R}\right|^{2}}$, then the fractional power from the absorber transmitted through to the radiometer will be

$$
\frac{\left|E_{A r}\right|^{2}}{\left|E_{A}\right|^{2}}=\frac{\left|E_{A}\right|^{2}-\left|E_{A r}\right|^{2}}{\left|E_{A}\right|^{2}}=1-\frac{\left|E_{A r}\right|^{2}}{\left|E_{A}\right|^{2}}=1-\frac{\left|E_{R_{r}}\right|^{2}}{\left|E_{R}\right|^{2}}
$$

where the subscripts $A, R, r$, and $t$ refer to the absorber, the receiver, the reflected component, and the transmitted component, respectively. The signal received by the radiometer is thus

$$
\begin{aligned}
\left|E_{R_{r}}\right|^{2}+\left|E_{A t}\right|^{2} & =\left|E_{A}\right|^{2}-\left|E_{A}\right|^{2} \frac{\left|E_{R_{r}}\right|^{2}}{\left|E_{R}\right|^{2}}+\left|E_{R_{r}}\right|^{2} \\
& =\left|E_{A}\right|^{2}-\frac{\left|E_{R_{r}}\right|^{2}}{\left|E_{R}\right|^{2}}\left(\left|E_{A}\right|^{2}-\left|E_{R}\right|^{2}\right) .
\end{aligned}
$$

The net reflection effect will therefore be proportional to the difference between the radiometer's broadcast temperature ( $\sim 80 \mathrm{~K})$ and the temperature of the target. 
For the $(\sim 4 \mathrm{~K})$ LHe target the decrease in target emission is small, and the sliding-short measurements have the emission source (the attenuator) between the reflection and the radioneter, so that the effect of reflections on the emitted power is negligible. For the dewar mock-up, however, the emission temperature is the ambient temperature, $288 \mathrm{~K}$, and the net reflection effect is then larger by a factor of $(288 \mathrm{~K}-80 \mathrm{~K}) /(4 \mathrm{~K}-80 \mathrm{~K})=-2.74$ from what it would be for a cold target. Hence the equivalent phase-dependent sine wave for the LHe target has an amplitude of $(2.0 \mathrm{~K}) / 2.74=0.73 \mathrm{~K}$. This is larger than the limit implied by the sliding-short tests, and may indicate that the dewar mock-up is not sufficiently accurate or that reflection in the antenna itself is more important than the (apparently larger) reflection within the radiometer. In any event, we'l" use the larger limit of $0.73 \mathrm{~K}$. Since the phase is unknown, the correction applied is 0 , with an error given by the root mean square of the sine wave, or $0.00 \pm 0.52 \mathrm{~K}$. Adding in the phase-independent term $\left(E_{0}{ }^{2} r_{2}{ }^{2}\right)$, the effect of reflections is to add $0.03 \pm 0.52 \mathrm{~K}$ to the antenna temperature of the target.

Thus, the total contribution from the cold calibration target is

$$
\mathrm{T}_{\mathrm{A}, \text { load }}=3.80 \pm 0.52 \mathrm{~K} \text {. }
$$




\section{$T_{\text {galaxy }}$}

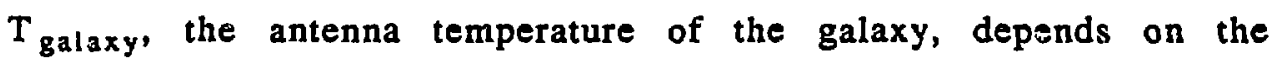
frequency of observation and on the part of the sky which is overhead when the CBR is being measured. We used this spatial and frequency dependence to help determine the contribution from the Galaxy.

The most direct way to determine $T_{\text {galaxy }}$ is to measure it using the $1.4 \mathrm{GHz}$ radiometer. As mentioned earlier, we did a galactic drift scan on August 10,1986 from 6:08 to 14:30 UT. During the scan, the radiometer automatically switched back and forth between two frequencies, $1.300 \mathrm{GHz}$ and $1.465 \mathrm{GHz}$. Also, a broadband microwave noise source was turned on for 16 out of every 32 seconds. After using the noise source signal to determine the radiometer gain, we can use the difference in sky signal at the two frequencies to determine the contribution from the Galaxy, based on an assumed spectral dependence.

Taking the galactic signal to be proportional to $\mathrm{v}^{-2.75}$ (Webster, 1974), where $v$ is the frequency, and assuming that the rest of the sky signal (principally $T_{A, C B R}$ ) is independent of frequency between 1.300 and $1.465 \mathrm{GHz}$, we have

$$
\mathrm{T}_{\text {galaxy, } 1.300 \mathrm{GHz}}=\left(\frac{1.465}{1.300}\right)^{2.75} \mathrm{~T}_{\text {galaxy,1.465 GHz}}
$$


so

$$
\mathrm{T}_{\text {galaxy, } 1.300 \mathrm{GHz}}-\mathrm{T}_{\text {galaxy, } 1.465 \mathrm{GHz}}=0.389 \mathrm{~T}_{\text {galaxy, } 1.465 \mathrm{GHz}} \text {. }
$$

Using the same power law to find the galactic signal at the frequency of observation, $1.410 \mathrm{GHz}$, we have

$$
\begin{aligned}
T_{\text {galaxy, } 1.410 \mathrm{GHz}} & =(1.465 / 1.410)^{2.75} \mathrm{~T}_{\text {galaxy, } 1.465 \mathrm{GHz}} \\
& =2.856\left(\mathrm{~T}_{\text {galaxy, } 1.300 \mathrm{GHz}}-\mathrm{T}_{\text {galaxy, } 1.465 \mathrm{GHz}}\right)
\end{aligned}
$$

During a galactic scan, the measured quantities are

$$
\begin{aligned}
S_{1.300} & =\left(T_{\text {galaxy, } 1.300 \mathrm{GHz}}+T_{C B R}+T_{S Y S, 1.300 \mathrm{GHz}}\right) / \mathrm{G}_{1.300} \\
\text { and } S_{1.465} & =\left(\mathrm{T}_{\text {galaxy,1.465 GHz}}+T_{C B R}+T_{S Y S, 1.465 \mathrm{GHz}_{2}}\right) / \mathrm{G}_{1.465}
\end{aligned}
$$

where $S$ refers to the radiometer output voltage, $T_{S Y S}$ is the system temperature of the radiometer, and $G$ refers to the gain. Gain drifts (largely due to temperature variation in the instrument) at 1.300 $\mathrm{GHz}$ are proportional to those at $1.465 \mathrm{GHz}$, so we can write the gain at $1.300 \mathrm{GHz}$ in terms of the gain at $1.465 \mathrm{GHz}$ and a constant, a.

$$
\mathrm{G}_{1.300}=\mathrm{aG}_{1.465}
$$

Solving for the galactic signal in terms of the measured quantities:

$$
\begin{aligned}
T_{\text {galaxy }} & \equiv T_{\text {galaxy, } 1.410 \mathrm{GHz}}=2.856\left(\mathrm{~T}_{\text {galaxy, } 1.300 \mathrm{GHz}}-T_{\text {galaxy, } 1.465 \mathrm{GHz}}\right) \\
& =2.856\left(\mathrm{aG}_{1.465} \mathrm{~S}_{1.300}-\mathrm{G}_{1.465} \mathrm{~S}_{1.465}-\mathrm{T}_{S Y S, 1.300}+\mathrm{T}_{S Y S, 1.465}\right)
\end{aligned}
$$




$$
=2.856\left[G_{1.465}\left(a S_{1.300}-S_{1.465}\right)-\Delta T_{S Y S}\right]
$$

where $\Delta \mathrm{T}_{S Y S}(-5 \mathrm{~K})$ is the difference in system temperatures at 1.300 and $1.465 \mathrm{GHz}$. From gain measurements discussed above, we know that the gain can vary by as much as several percent over the course of the night, and this gain drift, if uncorrected, would cause an error of $\delta G_{1.465}\left(a S_{1.300}-S_{1.465}\right)$, where $\delta G_{1.465}$ is the error in the gain at $1.465 \mathrm{GHz}$. For a $3 \%$ gain error and a (typical) value of $10 \mathrm{~K}$ for $G_{1.465}\left(a S_{1.300}-S_{1.465}\right)$, this produces an error of $0.3 \mathrm{~K}$. During the course of a galactic scan, the noise source is periodically turned on and off, introducing a known signal, and thus allowing a redetermination of $G_{1.465}$, provided that the noise signal itself remains constant throughout the scan. $\Delta \mathrm{T}_{S Y S}$ may also vary during the course of a (several hour) galactic scan.

On the nights when CBR measurements were being done, the system temperature at $1.410 \mathrm{GHz}$ drifted by several Kelvin during the course of a night. Figure 19 is a plot of $\mathrm{T}_{\text {galaxy }}$ versus position on the sky based on the single galactic scan performed on $8 / 10 / 86$. This single scan was taken during bad weather (see Chapter 4), and the variation evident in Figure 19, with no other scans available for cross-checking, makes it an unreliable measure of the galactic signal. Fortunately, we have other alternatives.

Using published maps of the galactic signal at $400 \mathrm{MHz}$ (Haslam et al. 1982), and scaling by the expected $v-2.75 \pm 0.1$ frequency dependence, 
Galactic Scan on $8 / 10 / 86$

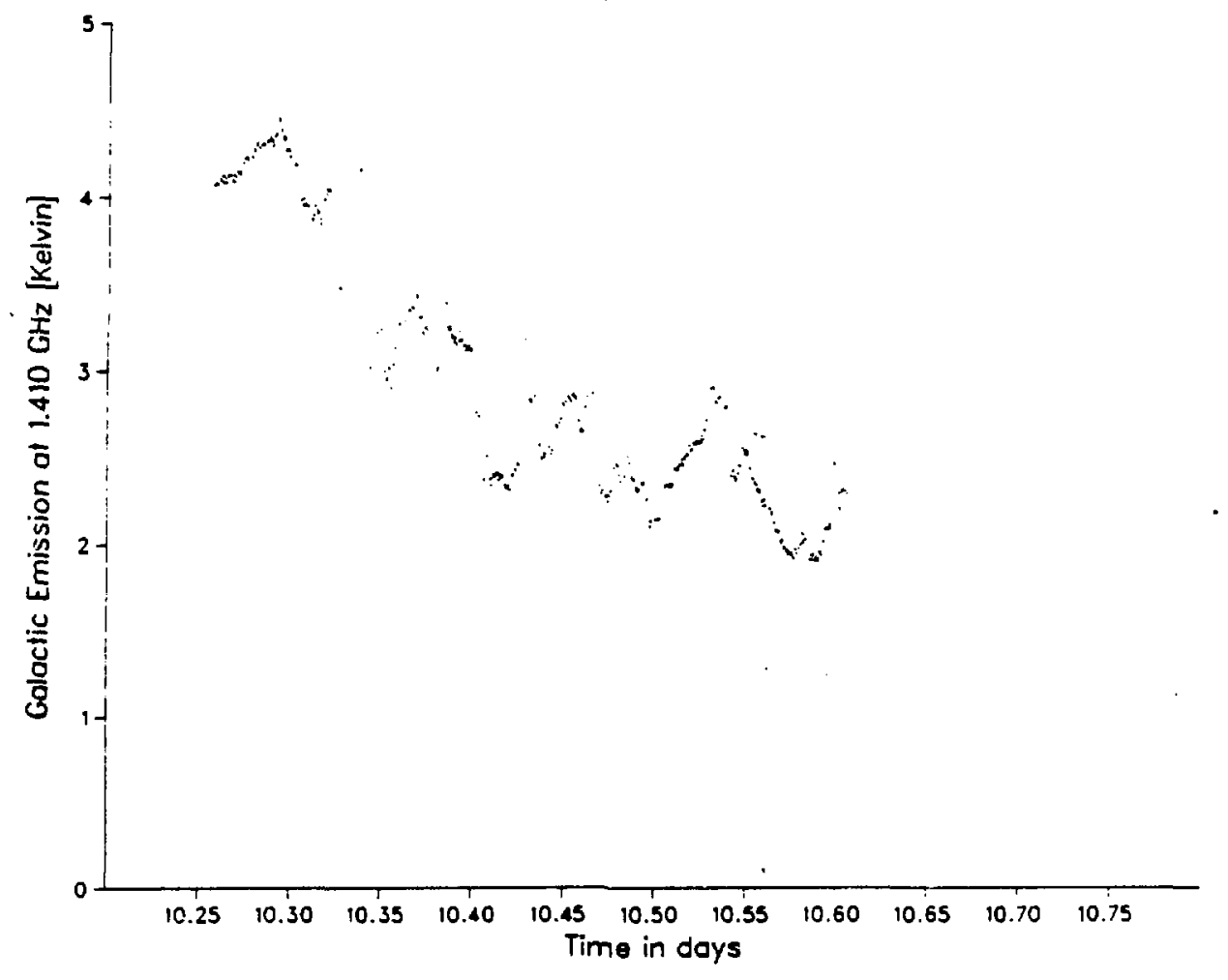

Figure 19. Galactic antenna temperature as determined by a single measurement scan on $8 / 10 / 86$. 
Signal From the Galaxy at $1.410 \mathrm{GHz}$ of 38 Degrees Declination

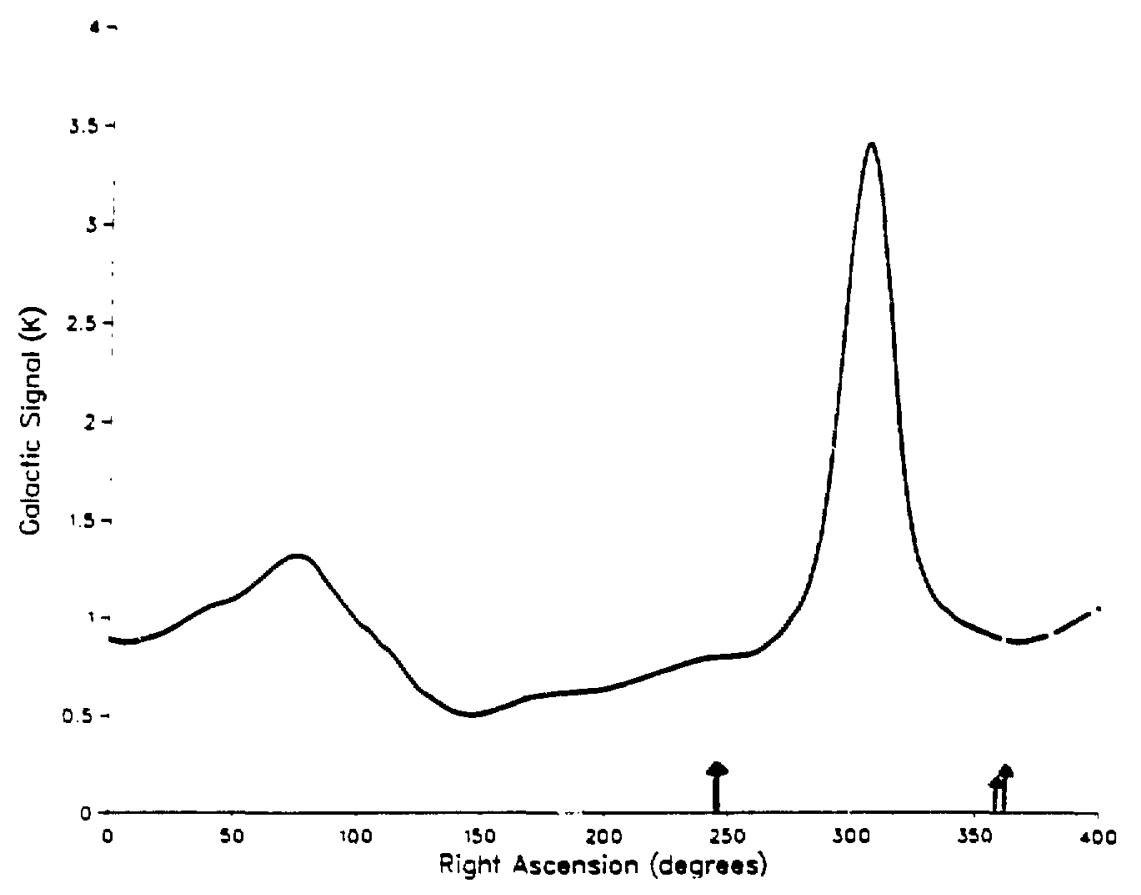

Figure 20. Galactic signal as a function of R.A. at $1.410 \mathrm{GHz}$ as seen from latitude 34. Extrapolated from published measurements at 400 MHz. 
we can calculate the galactic signal at $1.410 \mathrm{GHz}$ as a function of position on the sky. Figure 20 shows the predicted signal as seen by the $1.410 \mathrm{GHz}$ radiometer (looking vertically from our observing site) as the Earth's rotation varies the Right Ascension of observation. The arrows indicate times when the radiometer was taking data with the LHe target.

Data were taken (in roughly equal numbers) at Right Ascensions of 15.87-16.70, 23.89-00.72, and 23.23-00.06. Using the model described above, the weighted average ratio of the galactic signal at these positions to the peak galactic signal is 0.243. Brief measurements were made on White Mountain at $1.410 \mathrm{GHz}$ on $8 / 8 / 86$ and $8 / 9 / 86$ while the galactic signal passed through maximum. The measured average difference between the peak signal and the signal at other times was $2.2 \pm 0.3 \mathrm{~K}$, so solving for the galactic signal during $C B R$ measurements, $T_{\text {galaxy }}=0.71 \pm 0.10 \mathrm{~K}$.

We can verify this result by comparing with a measurement recently made by Giorgio Sironi (1987) at $0.60 \mathrm{GHz}$. According to his preliminary result (private communication, 12/11/86), the galactic signal in a nearby (within 20 degrees) region of the sky, at the same galactic latitude, is $T_{\text {galaxy, } 0.60 \mathrm{GHz}}=7 \pm 1 \mathrm{~K}$. Scaling by $v-2.75 \pm 0.1$, we find that at $1.410 \mathrm{GHz}$ we have $\mathrm{T}_{\text {galaxy }}=0.67 \pm 0.15 \mathrm{~K}$, which is consistent with the other estimate. The estimate of the galactic signal used for substitution in Equation 8 is $T_{\text {galaxy }}=0.71 \pm 0.10 \mathrm{~K}$. 


\section{$\Delta T_{\text {offset }}$}

The central assumption of our measurement is that when the radiometer alternates between viewing the sky and viewing the LHe target, the only changes in signal are those due to the difference in targets. Because we move the radiometer from one position to another in viewing the different targets, this assumption must be tested. The radiometer operates within gravitational and electromagnetic fields which are directional in nature. Conceivably, changes in the stresses on the electronic components could induce small changes in their positions, which could in turn slightly change the gain of the radiometer. Similarly, rotating the radiometer within the Earih's magnetic field could have an effect on the magnetically sensitive components (particularly the YIG filter), again changing slightly the gain of the radiometer.

Regardless of the causes, any change in the radiometer gain as a function of orientation can be detected by performing a "flip test," in which a target is attached to the horn of the radiometer, and the radiometer is repeatedly rotated, with the target attached. By measuring the amplitude (if any) of the resultant square-wave radiometer output, we can determine the extent to which rotating the radiometer changes its gain. Such a flip test was performed on $8 / 6 / 86$, and the results are discussed below.

The ambient-temperature target was the only stable target capable 
of staying attached to the radiometer while being inverted, and was used as a target when doing flip tests. We soon discovered, however, that slight deformations in the shape of the target (achieved by pushing on the back of the metal casing) caused significant changes in the radiometer signal. Return-loss measurements with the directional coupler and the spectrum analyzer showed a power reflection coefficient of $-30 \mathrm{~dB}$. Adding stiffeners to the target and rearranging the microwave absorber to reduce reflections made the target more stable under gravitational stress, but still left a residual effect.

The vertical flip test done on $8 / 6 / 86$ showed a $0.35 \mathrm{~K}$ effect at 1.410 $\mathrm{GHz}$ and a $-0.15 \mathrm{~K}$ effect at $1.465 \mathrm{GHz}$. The previously mentioned problems with the ambient target, as well as the strong frequency dependence of the effect, argue that the "flip offset" seen is actually caused by a change in the reflection of the ambient-temperature target, masking any potential change in the radiometer gain. Using the signal seen in the flip test as an upper limit on changes in the radiometer gain due to gravitational stress, we thus have a possible flip offset when viewing the ambient target of $0.35 \mathrm{~K}$. The signal with the ambient target is $280 \mathrm{~K}$ plus the $80 \mathrm{~K}$ system temperature, or $360 \mathrm{~K}$, and the signal when looking at LHe is $\sim 85 \mathrm{~K}$, so the possible effect of such gain changes on the radiometer signal is $(0.35 \mathrm{~K})(85 / 360)=0.083 \mathrm{~K}$.

A change in system temperature would not scale in this fashion, but 
one expects such a change to be accompanied by a corresponding change in gain. There are no active components upstream of the first amplifier, and a change in any component downstream from the first amplifier should result in a change in gain. A change in the insertion loss of the antenna would go undetected in a flip test with the ambient-temperature target, but the antenna is rigidly constructed and securely attached to the receiver to minimize any change due to gravitational stress.

We therefore assume that the effect (if any) or rotating the radiometer is a change in gain, and $\Delta \mathrm{T}_{\text {offset }}=0.00 \pm 0.083 \mathrm{~K}$. 


\section{$\mathbf{T}_{\text {ground }}$}

Because the surrounding terrain is at a temperature of $\sim 300 \mathrm{~K}$, and the sky signal is only $-4 \mathrm{~K}$, the power radiated by the ground is nearly 100 times greater than the signal we're trying to look at. Accordingly, the antenna must have very low gain at angles below the local horizon. The antenna is discussed in detail in a paper published elsewhere (reproduced as Appendix $C$ ), but Figure 21 shows the measured antenna gain as a function of angle at $1.410 \mathrm{GHz}$.

At its highest point, the local horizon for our observing site is 67 degrees from the zenith. Taking the antenna gain to be cylindrically symmetric with the gain as measured in the H-plane (Appendix C), and treating the effective ground temperature as $300 \mathrm{~K}$ for zenith angles greater than 60 degrees, and $0 \mathrm{~K}$ for zenith angles less than 60 degrees I have numerically integrated the antenna response, and conclude that (under these conservative assumptions) the signal received from the ground is $\mathrm{T}_{\text {ground }}=0.009 \pm 0.008 \mathrm{~K}$. Clearly the assumptions of beam symmetry and uniform horizon height are not valid, but they are conservative, and the resulting value for $\mathrm{T}_{\text {ground }}$ is so small compared to other sources of error that further analysis is not justified. 


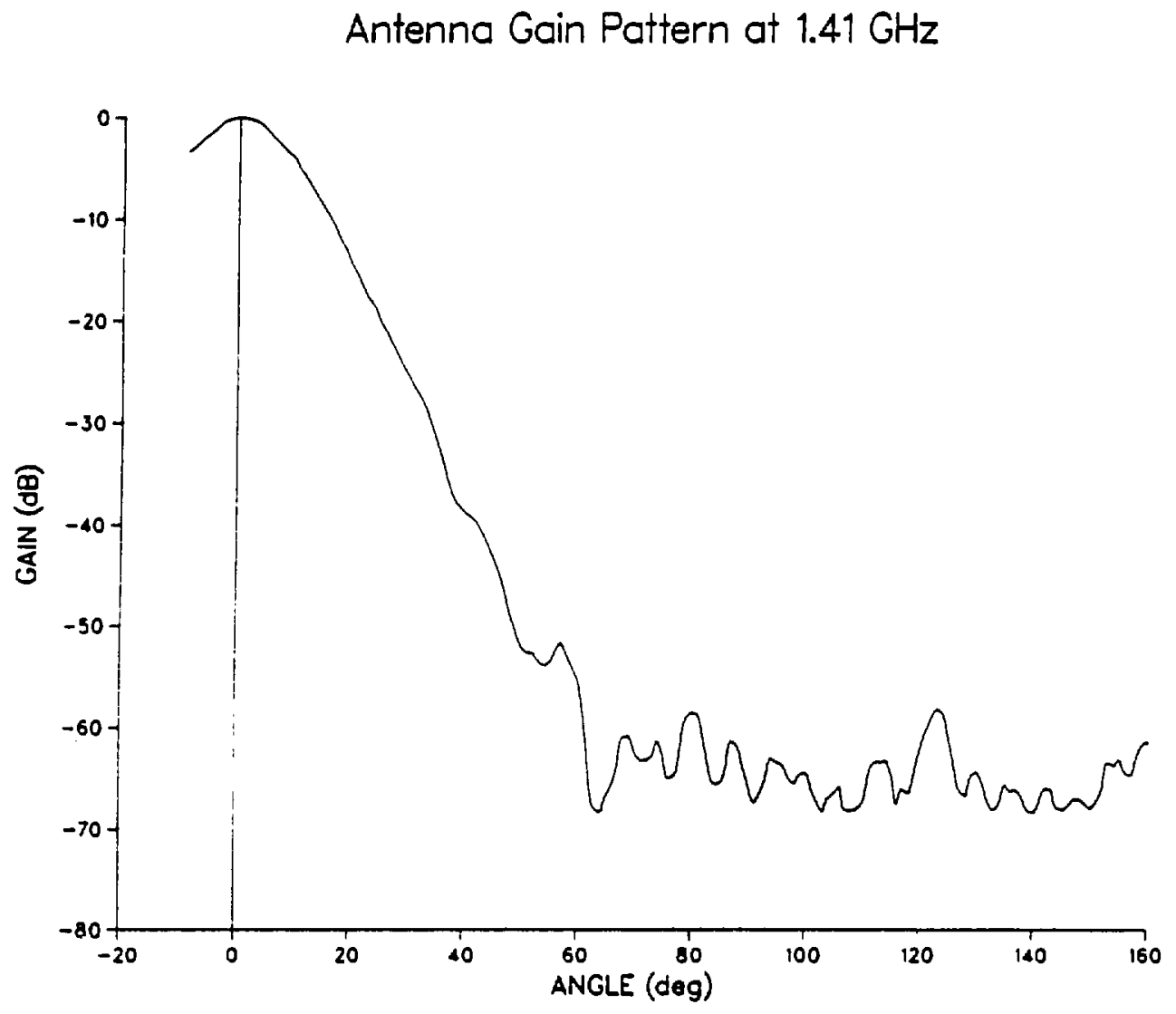

Figure 21. Gain pattern of the $1.4 \mathrm{GHz}$ antenna at $1.410 \mathrm{GHz}$. 


\section{Tatmosphere}

In the past, with other radiometers, we have measured the microwave emission from the atmosphere by tilting the radiometer to observe different zenith angles, and fitting the signal observed to a model of the atmosphere. If the atmosphere were composed of uniform planar layers, the amount of atmosphere traversed by the beam would be proportional to the secant of the zenith angle. For the actual atmosphere, the analysis is slightly more complicated, but has been worked out numerous times (e.g. Smoot et al. 1987).

Unfortunately, the $1.4 \mathrm{GHz}$ radiometer was too big and unwieldy to perform zenith scans of this type, so we were forced to rely on measurements made at other frequencies. At the same time the 1.4 $\mathrm{GHz}$ radiometer was taking data, radiometers at $3.7 \mathrm{GHz}$ and $10 \mathrm{GHz}$ were making atmospheric measurements from the same highaltitude site (De Amici et al. 1987, Kogut et al. 1987). We have extrapolated these atmospheric measurements from $3.7 \mathrm{GHz}$ to 1.410 $\mathrm{GHz}$, based on published models of the frequency dependence of atmospheric absorption (Waters 1976, Rosenkranz 1975, Liebe 1981, Costales 1985).

According to the atmospheric model (discussed in more detail in Appendix B), the atmospheric emission at $1.410 \mathrm{GHz}$ ranges from $97 \%$ of the emission at $3.7 \mathrm{GHz}$ (a completely dry atmosphere) to $93 \%$ of the emission at $3.7 \mathrm{GHz}$ (an atmosphere with $8 \mathrm{~mm}$ of water vapor). 
Thus the ratio of the atmospheric emission at $1.410 \mathrm{GHz}$ to the emission at $3.7 \mathrm{GHz}$ is $0.95 \pm 0.02$.

The antenna temperature of the vertical atmosphere at $3.7 \mathrm{GHz}$ was measured to be $0.875 \pm 0.073 \mathrm{~K}$, so the antenna temperature of the vertical atmosphere at $1.410 \mathrm{GHz}$ is $\mathrm{T}_{\text {atmosphere }}=0.831 \pm 0.075 \mathrm{~K}$. 
Summing all the terms from the preceding chapter, adding the errors in quadrature, we arrive at the antenna temperature of the CBR at $1.410 \mathrm{GHz}$ :

$$
T_{A, C B R}=G\left(S_{\text {zenith }}-S_{\text {load }}\right)
$$

$$
\begin{gathered}
-T_{\text {galaxy }}-T_{\text {atmosphere }}-T_{\text {ground }}-\Delta T_{\text {offset }}+T_{A, \text { load }} \\
=-0.06 \pm 0.03 \mathrm{~K}-0.71 \pm 0.10 \mathrm{~K}-0.831 \pm 0.075 \mathrm{~K} \\
-0.009 \pm 0.008 \mathrm{~K}-0.000 \pm 0.083 \mathrm{~K}+3.80 \pm 0.52 \mathrm{~K} \\
\mathrm{~T}_{\mathrm{A}, \mathrm{CBR}}=2.19 \pm 0.54 \mathrm{Kelvin}
\end{gathered}
$$

Converting to brightness temperature (Equation 4), we have the final result:

$$
\mathrm{T}_{\mathrm{CBR}}=2.22 \pm 0.55 \text { Kelvin }
$$

Figure 22 is a plot of this result and other recent measurements of $T_{C B R}$ at nearby frequencies.

The purpose of this measurement was to see if the CBR deviates from a Planckian spectrum. In the next chapter, I will use this measurement, along with other recent measurements of the intensity of the CBR (see Table 5), to look for such "distortions" of the spectrum of the CBR, and I will interpret the results of that analysis. 


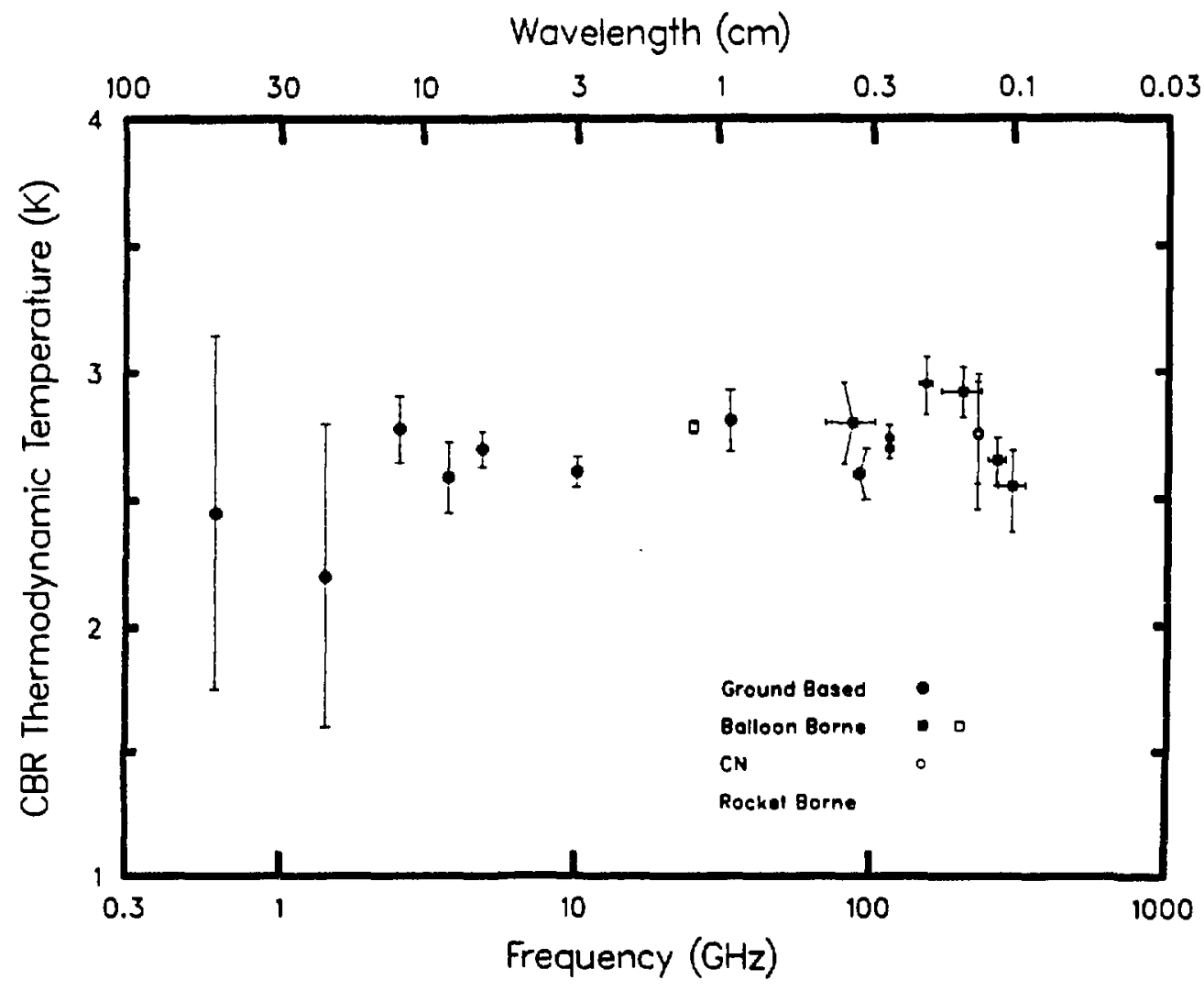

Figure 22. Measurements of the temperature of the Cosmic Background Radiation as a function of frequency. 


\begin{tabular}{|llll|}
\hline Reference & $\begin{array}{c}\text { Wavelength } \\
\text { (cm) }\end{array}$ & $\begin{array}{c}\text { Frequency } \\
\text { (GHz) }\end{array}$ & $\begin{array}{c}\text { TCBR } \\
(\mathrm{K})\end{array}$ \\
\hline Sironi et al., 1986 & 50.0 & 0.6 & $2.45 \pm 0.70$ \\
\hline Levin (this work) & 21.3 & 1.41 & $2.22 \pm 0.55$ \\
\hline $\begin{array}{l}\text { Sironi } \\
\text { (Smoot et al.,1985b) }\end{array}$ & 12.0 & 2.5 & $2.78 \pm 0.13$ \\
\hline De Amici et al., 1987 & 8.2 & 3.66 & $2.59 \pm 0.14$ \\
\hline Mandolesi et al., 1986 & 6.3 & 4.75 & $2.70 \pm 0.07$ \\
\hline Kogur et al., 1987 & 3.0 & 10.0 & $2.60 \pm 0.06$ \\
\hline Johnson and & 1.2 & 24.8 & $2.783 \pm 0.025$ \\
\hline Wilkinson, 1986 & & & \\
\hline De Amici & 0.909 & 33.0 & $2.81 \pm 0.12$ \\
(Smoot et al., 1985b) & 0.333 & 90.0 & $2.60 \pm 0.10$ \\
\hline Bersanelli & & & \\
(Smoot et al., 1987) & & 113.6 & $2.70 \pm 0.04$ \\
\hline Meyer \& Jura, 1985 & 0.264 & 227.3 & $2.76 \pm 0.20$ \\
\hline Meyer \& Jura, 1985 & 0.132 & 113.6 & $2.74 \pm 0.05$ \\
\hline Crane et al., 1986 & 0.264 & 227.3 & $2.75 \pm 0.24$ \\
\hline & 0.132 & 85.5 & $2.80 \pm 0.16$ \\
\hline Peterson, Richards, & 0.351 & 151 & $2.95 \pm 0.11$ \\
\& Timusk, 1985 & 0.198 & 203 & $2.92 \pm 0.10$ \\
\hline & 0.148 & 264 & $2.65 \frac{+0.09}{-0.10}$ \\
& 0.114 & 299 & $2.55 \frac{+0.14}{-0.18}$ \\
\hline
\end{tabular}

Table 5. Recent measurements of $T_{C B R}$. 
Having presented data regarding the spectrum of the CBR, in this chapter I will interpret that data in the context of theory. I will begin by sketching a theoretical outline of how an early energy release would have caused distortions in the CBR spectrum, and then I'll discuss how data and theory fit together.

As mentioned in the Introduction, a simple model of the Big Bang predicts that the CBR spectrum is Planckian, so that on a plot like Figure 22 it would appear as a horizontal straight line. Energy releases early in the history of the universe, however, would cause distortions from this blackbody spectrum. The particular shape and size of the distortion depends on the timing, size, and character of the energy release.

At very early times, when the universe was both dense and hot, there were numerous ways for particles ar. photons to interact on time scales short compared to the expansion time of the universe, so the matter and light vere in thermal equilibrium, and any perturbation in the CBR spectrum was quickly erased. As the universe cooled, fewer interaction processes remained within reach of the energy levels involved. At early enough times, the process of double Compton scattering $\left(\gamma+\mathrm{e}^{-} \leftrightarrow \gamma+\gamma+\mathrm{e}^{-}\right)$was strong enough to completely thermalize arbitrarily large distortions. Denoting $z_{D C}$ as the redshift beyond which double Compton scattering removed all distortions, Danese and De Zotti (1982) find $z_{D C}=2 \times 10^{6} h_{0}-2 / 3 \Omega_{b}-1 / 3$. Here $h_{0}$ is defined as the Hubble constant divided by 50 
$\mathrm{km} / \mathrm{sec} / \mathrm{MPc}$, and $\Omega_{\mathrm{b}}$ is the baryon density of the universe divided by the critical density.

At redshifts small enough for double Compton scattering to be unimportant $\left(z<\sim 6 \times 10^{5}\right)$, the dominant interaction mechanism between matter and radiation is ordinary Compton scattering. While ordinary Compton scattering can restore thermal equlibrium, it cannot establish a Planckian spectrum at the higher temperature corresponding to an increase in energy, because ordinary Compton scattering does not produce new photons. Instead, existing photons are shifted to higher frequencies so that they carry more energy.

The average rate of fractional energy gain for a photon through Compton scattering is

$$
\alpha_{0}(z)=\sigma_{T} n_{e}(z) c \frac{k T_{e}(z)}{m_{e} c^{2}}
$$

where $\sigma_{T}$ is the Thomson scattering cross-section, $n_{e}(z)$ is the electron density, $c$ is the speed of light, $k$ is Boltzmann's constant, $T_{e}(z)$ is the electron temperature, and $m_{e}$ is the mass of an electron. The ratio of electron kinetic energy to rest mass determines the efficiency of an individual scattering, so $\alpha_{0}(z)$ is just the product of the efficiency of each scattering times the number of scatterings per unit time. To find the change in a photon's energy from initial time $t$ to the present, we just integrate. Following the notation of Zel'dovich and Sunyaev (1969), we call this integral $y(z)$, where 


$$
y(z)=-\int_{0}^{t(z)} \alpha_{0}(t) d t=-\int_{0}^{z(t)} \alpha_{0}(z) \frac{d t}{d z} d z
$$

Defining $z_{a}$ by $y\left(z_{a}\right)=1$, we have $z_{a}=3800 h_{0}^{-1} \Omega_{b}^{-1 / 2}$. For energy release at redshifts greater than $z_{2}(y>1)$, the Universe is optically thick to Compton scattering. Compton scattering preserves photon number, so it creates a Bose-Einstein spectrum. Bremsstrahlung creates low-energy photons, resulting in a Planckian spectrum at low frequencies. The result is a spectrum with a frequency-dependent chemical potential, $\mu(x)$, so that at a redshift of approximately $z_{a}$, the photon occupation number, $\eta$, is

$$
\eta(x)=\frac{1}{e^{x+\mu(x)}-1}
$$

where $x$ is the dimensionless frequercy, hv/kT. Hence the briglitness temperature is

$$
T_{B}(x)=\frac{x T}{x+\mu(x)}
$$

$\mu(x)$ is approximately given by $\mu(x)=\mu_{0} \exp \left(2 x_{1} / x\right.$ ) (Sunyaev and Zel'dovich 1970; Danese and De Zotti 1980), where $x_{1}$ is the transition frequency between the Bose-Einstein and Planckian spectra. The transition frequency is the highest frequency for which bremsstrahlung still produces photons faster than Compton scattering can remove them by shifting them to higher frequencies. 
The brightness temperature goes through a minimum at a frequency of $2 x_{1}$. Sunyaev and Zel'dovich (1970) calculate $x_{1}=$ $50[g(x)]^{1 / 2} \Omega_{b}^{1 / 2} z^{-3 / 4}$, where $g$ is the Gaunt factor. Later on, bremsstrahlung fills in some of the dip in the spectrum. If there were no Compton scattering, bremsstrahlung could fill in the spectrum up to a frequency, $x_{B}$, at which the optical depth to freefree absorption is unity. Danese and De Zotti (1980) calculate $x_{B}=5.3 \times 10^{;}[g(x)]^{1 / 2} \Omega_{b}\left\{\left(z+z_{e q}\right)^{1 / 2}-\left(z_{e q}+z_{\text {tec }}\right)^{1 / 2}\right\}^{1 / 2}$, where $z_{\text {eq }}$ is the redshift at which the matter energy density equals the radiation energy density and $z_{\text {rec }}$ is the redshift at which recombination takes place, when the ions and electrons join to form atoms. After recombination, the universe is essentially transparent to bremsstrahlung.

For energy added to the radiation before $z_{a}$, the universe is optically thick to Compton scattering, so the resultant spectrum is independent of the details of the energy injection. The chemical potential is approximately

$$
\mu_{0}=1.4 \frac{\delta U}{U_{0}}
$$

where $U_{0}$ is the energy in the unperturbed $C B R$ and $\delta U$ is the energy added. Note that the spectrum is also independent of the time of energy release, provided that it takes place before $z_{a}$ and after $z_{D C}$.

For energy releases after $z_{a}$, the spectrum is still affected, even 
though Compton scattering doesn't have time to establish a BoseEinstein spectrum. Denoting the redshift at which energy is added by $z_{h}$, we can define a parameter $u$ :

$$
u=-\int_{0}^{t\left(z_{h}\right)} \alpha_{0}(t)\left[\left(T_{e}-T_{R}\right) / T_{e}\right] d t=\int_{0}^{z_{h}} \alpha_{0}(z)\left[\left(T_{e}-T_{R}\right) / T_{e}\right](d t / d z) d z
$$

where $T_{e}$ is the electron temperature and $T_{R}$ is the unperturbed radiation temperature (Sunyaev and Zel'dovich 1980).

To first order in ux, the occupation number due to Compton scatterings is then

$$
\eta_{C}(x, u)=\frac{1+\frac{u x e^{x}}{e^{x}-1}(x / \tanh (x / 2)-4)}{e^{x-1}}
$$

When bremsstrahlung is taken into account, we get (Danese and De Zotti, 1982)

$$
\eta=\eta_{C} e^{-y_{B}\left(x_{e}\right)}+\frac{1-e^{-y_{B}\left(x_{e}\right)}}{e^{x_{e}}-1}
$$

where $\eta_{C}$ comes from Equation 24 above. From conservation of energy, the fractional energy added to the CBR is 


$$
\frac{\Delta \mathrm{U}}{\mathrm{U}_{0}}=\mathrm{e}^{4 \mathrm{u}}-1
$$

(Sunyaev and Zel'dovich 1980).

If the energy release takes place after recombination $\left(z<z_{R}=1000\right)$, when the universe has cooled enough for protons and electrons to combine and make hydrogen atoms, then the situation changes again. Neutral atoms have a much lower cross section for interacting with the cosmic microwave background radiation, so the universe became largely transparent at the time of recombination. Any uniform energy release, provided it was not enough to re-ionize the matter, would have very little effect on the spectrum of the CBR. Any general energy release large enough to re-ionize all the matter would have more energy than the CBR itself, and can be easily ruled out by the observed spectrum of the CBR. On the other hand, heating only part of the matter enough for re-ionization would introduce a distortion in the spectrum of the $C B R$ without requiring such large energy releases. The shape of such a distortion would be the same as that predicted for energy releases taking place before recombination, with the bremsstrahlung term becoming negligible. (Note that bremsstrahlung is proportional to the matter density squared, while Compton scattering is proportional to the first power of the matter density, so that if only a small part of the matter is ionized, Compton scattering is much more important than bremsstrahlung.)

After a redshift of $z \approx 8$, the number of scatterings per photon is 
small, so it's possible to have energy releases to the matter larger than the corresponding energy added to the CBR. The spectral distortions remain the same in this regime, but it must be remembered that in this case $\Delta \mathrm{U} / \mathrm{U}_{0}$ refers to the energy which actually is added to the CBR, rather than to the total energy released to the matter.

In summary then, we can divide the history of the universe into the 5 regimes discussed below.

(1) Before $\mathrm{z}_{\mathrm{DC}}\left(=2 \times 10^{6} \mathrm{~h}_{0}-2 / 3 \Omega_{\mathrm{b}}^{-1 / 3}\right)$, double Compton scattering re-established a Planckian spectrum faster than any perturbations could alter the CBR.

(2) After $z_{D C}$ but before $z_{a}$, single Compton scattering thermalized energy releases, producing a Bose-Einstein spectrum modified by the bremsstrahlung which took place later. Such a spectrum can be characterized by a single parameter, $\mu_{0}=1.4\left(\delta \mathrm{U} / \mathrm{U}_{0}\right)$.

(3) From $z_{a}$ to recombination $\left(z_{R} \approx 1000\right)$, the spectrum is not completely Comptonized, so that a Bose-Einstein spectrum is not formed, and the spectrum (a combination of bremsstrahlung and Comptonization that depends on when the energy release takes place) is characterized by the redshift of energy release, $z_{h}$, and the parameter $u$, with $\delta U / U_{0}=e^{4 u}-1=4 u$.

(4) After recombination but before $z-8$, the shape of the spectral 
distortion in the CBR is the same as for $z \approx z_{R}$ but the only energy detected is energy in ionized matter, such as ionized clouds which could be formed from more localized energy releases.

(5) From $z=z_{R}$ until the present, the spectral distortions in the CBR have the same form as in the previous two regimes, but $\delta U / U_{0}$ refers to the energy actually transferred to the CBR, which is less than the energy in the matter.

Using the distortion models described above, I have used the existing measurements of the CBR spe rum to place limits on possible energy releases at various times. Table 5 is a list of all the recent measurements of the CBR intensity. After choosing a time of energy release, assuming a value for $\Omega_{b}$, picking the size of the energy release, and assuming a value for the unperturbed CBR temperature, one can use the models to predict the spectrum of the CBR. By summing the squares of the differences between the $C B R$ measurements in Table 5 and the prediction in the usual way, one arrives at a value of $\chi^{2}$ for that set of parameters.

Figure 23 shows contours of constant $\chi^{2}$ in the $\mu_{0}-\mathrm{T}_{0}$ and $\mathrm{u}-\mathrm{T}_{0}$ planes for various values of $\Omega_{b}$ and $z_{h} . T_{0}$ is the unperturbed $C B R$ temperatus. Note that negative values of $u$ and $\mu_{0}$ are allowed, even though they are not physically meaningful. For given values of $z_{h}$ and $\Omega_{\mathrm{b}}$, the $95 \%$ confidence level corresponds to the contour with $\chi^{2}$ greater by 4 than the minimum $\chi^{2}$. Table 6 gives best-fit and 


\begin{tabular}{|c|c|c|c|c|c|c|c|c|}
\hline \multirow{2}{*}{$\Omega_{b}$} & \multirow[t]{2}{*}{$z_{h}$} & & Best & \multicolumn{3}{|c|}{ Largest $\mu_{0}{ }^{*}$} & \multirow{2}{*}{$\begin{array}{l}\text { Largest } \\
T_{0}\end{array}$} & \multirow{2}{*}{$\begin{array}{c}T_{0}^{*} \\
\mu_{0}\end{array}$} \\
\hline & & $\mathrm{T}_{0}$ & $\mu_{0}$ & $x^{2}$ & $T_{0}$ & $\mu_{0}$ & & \\
\hline & & & $4.27 \times 10^{-3}$ & 21.69 & 2.805 & $14.10 \times 10^{-3}$ & 2.815 & $12.70 \times 10^{-3}$ \\
\hline
\end{tabular}

\begin{tabular}{|c|c|c|c|c|c|c|c|c|}
\hline \multirow[t]{2}{*}{$\Omega_{b}$} & \multirow[t]{2}{*}{$\mathbf{z h}$} & \multicolumn{2}{|r|}{ Best Fit } & \multicolumn{3}{|c|}{ Largest $U^{*}$} & \multicolumn{2}{|c|}{ Largest $T_{0}{ }^{*}$} \\
\hline & & $\mathrm{T}_{0}$ & $\mathbf{U}$ & $x^{2}$ & $\mathbf{T}_{\mathbf{0}}$ & $\mathbf{U}$ & $T_{0}$ & U \\
\hline 1.0 & 40000 & 2.724 & $-6.03 \times 10^{-3}$ & 20.33 & 2.746 & $2.05 \times 10^{-3}$ & 2.763 & $-0.45 \times 10^{-3}$ \\
\hline 1.0 & 10000 & 2.715 & $-7.28 \times 10^{-3}$ & 21.06 & 2.758 & $4.66 \times 10^{-3}$ & 2.768 & $3.22 \times 10^{-3}$ \\
\hline 1.0 & 4000 & 2.707 & $-8.48 \times 10^{-3}$ & 21.42 & 2.773 & $7.47 \times 10^{-3}$ & 2.778 & $6.64 \times 10^{-3}$ \\
\hline 1.0 & $<1000$ & 2.715 & $-4.54 \times 10^{-3}$ & 21.28 & 2.809 & $12.82 \times 10^{-3}$ & 2.809 & $12.17 \times 10^{-3}$ \\
\hline 0.1 & 100000 & 2.713 & $-6.56 \times 10^{-3}$ & 21.98 & 2.791 & $11.18 \times 10^{-3}$ & $2: 796$ & $10.46 \times 10^{-3}$ \\
\hline 0.1 & 40000 & 2.715 & $-6.00 \times 10^{-3}$ & 22.08 & 2.795 & $11.96 \times 10^{-3}$ & 2.800 & $11.20 \times 10^{-3}$ \\
\hline & 10000 & 2.718 & $-5.26 \times 10^{-3}$ & 22.19 & 2.800 & $12.93 \times 10^{-3}$ & 2.805 & $12.12 \times 10^{-3}$ \\
\hline 1 & 4000 & 2.719 & $-4.98 \times 10^{-3}$ & 22.24 & 2.807 & $11.54 \times 10^{-3}$ & 2.807 & $11.33 \times 10^{-3}$ \\
\hline 0.1 & $<1000$ & 2.721 & $-4.54 \times 10^{-3}$ & 22.28 & 2.809 & $12.82 \times 10^{-3}$ & 2.809 & $12.17 \times 10^{-3}$ \\
\hline
\end{tabular}

*95\% Confidence Level

Table 6. Fits of the data to various models of distortions of the CBR spectrum. 
distortion in the CBR is the same as for $z \approx z_{R}$ but the only energy detected is energy in ionized matter, such as ionized clouds which could be formed from more localized energy releases.

(5) From $z=z_{R}$ until the present, the spectral distortions in the CBR have the same form as in the previous two regimes, but $\delta U / U_{0}$ refers to the energy actually transferred to the CBR, which is less than the energy in the matter.

Using the distortion models described above, I have used the existing measurements of the CBR spectrum to place limits on possible energy releases at various times. Table 5 is a list of all the recent measurements of the CBR intensity. After choosing a time of energy release, assuming a value for $\Omega_{\mathrm{b}}$, picking the size of the energy release, and assuming a value for the unperturbed CBR temperature, one can use the models to predict the spectrum of the CBR. By summing the squares of the differences between the CBR measurements in Table 5 and the prediction in the usual way, one arrives at a value of $\chi^{2}$ for that set of parameters.

Figure 23 shows contours of constant $\chi^{2}$ in the $\mu_{0}-T_{0}$ and $u-T_{0}$ planes for various values of $\Omega_{b}$ and $z_{h}$. $T_{0}$ is the unperturbed CBR temperature. Note that negative values of $\mathbf{u}$ and $\mu_{0}$ are allower, even though they are not physically meaningful. For given values of $z_{h}$ and $\Omega_{\mathrm{b}}$, the $95 \%$ confidence level corresponds to the contour with $\chi^{2}$ greater by 4 than the minimum $\chi^{2}$. Table 6 gives best-fit and 


\begin{tabular}{|c|c|c|c|c|c|c|c|}
\hline \multirow{2}{*}{$\Omega_{\mathrm{b}}$} & \multirow[t]{2}{*}{$\mathbf{z h}$} & & Best & & \multirow{2}{*}{ Largest $\mu_{0}^{*}$} & \multirow{2}{*}{$\begin{array}{l}\text { Largest } \\
\mathrm{T}_{\mathbf{0}}\end{array}$} & \multirow{2}{*}{$\begin{array}{c}\mathbf{T}_{0}{ }^{*} \\
\mu_{0}\end{array}$} \\
\hline & & $T_{0}$ & $\mu_{0}$ & $x^{2}$ & & & \\
\hline 0.0 & $>40000$ & 2.760 & $4.27 \times 10^{-3}$ & 21.69 & $2.80514 .10 \times 10^{-3}$ & 2.815 & $12.70 \times 10^{-3}$ \\
\hline 0.1 & $>100000$ & 2.755 & $1.97 \times 10^{-3}$ & 21.22 & $2.780 \quad 5.63 \times 10^{-3}$ & 2.815 & $4.62 \times 10^{-3}$ \\
\hline
\end{tabular}

\begin{tabular}{|c|c|c|c|c|c|c|c|c|}
\hline \multirow[t]{2}{*}{$\Omega_{b}$} & \multirow[t]{2}{*}{$\mathbf{2 h}$} & \multicolumn{2}{|r|}{ Best Fit } & \multirow[b]{2}{*}{$x^{2}$} & \multicolumn{2}{|c|}{ Largest $\mathbf{U}^{*}$} & \multicolumn{2}{|c|}{ Largest $T_{0}{ }^{*}$} \\
\hline & & $T_{0}$ & $\mathbf{U}$ & & $\mathbf{T}_{0}$ & $\mathbf{U}$ & $\mathrm{T}_{\mathbf{0}}$ & U \\
\hline 1.0 & 40000 & 2.724 & $-6.03 \times 10^{-3}$ & 20.33 & 2.746 & $2.05 \times 10^{-3}$ & 2.763 & $-0.45 \times 10^{-3}$ \\
\hline 1.0 & 10000 & 2.715 & $-7.28 \times 10^{-3}$ & 21.06 & 2.758 & $4.66 \times 10^{-3}$ & 2.768 & $3.22 \times 10^{-3}$ \\
\hline 1.0 & 4000 & 2.707 & $-8.48 \times 10^{-3}$ & 21.42 & 2.773 & $7.47 \times 10^{-3}$ & 2.778 & $6.64 \times 10^{-3}$ \\
\hline 1.0 & $<1000$ & 2.715 & $-4.54 \times 10^{-3}$ & 21.28 & 2.809 & $12.82 \times 10^{-3}$ & 2.809 & $12.17 \times 10^{-3}$ \\
\hline 0.1 & 100000 & 2.713 & $-6.56 \times 10^{-3}$ & 21.98 & 2.791 & $11.18 \times 10^{-3}$ & 2.796 & $10.46 \times 10^{-3}$ \\
\hline 0.1 & 40000 & 2.715 & $-6.00 \times 10^{-3}$ & 22.08 & 2.795 & $11.96 \times 10^{-3}$ & 2.800 & $11.20 \times 10^{-3}$ \\
\hline 0.1 & 10000 & 2.718 & $-5.26 \times 10^{-3}$ & 22.19 & 2.800 & $12.93 \times 10^{-3}$ & 2.805 & $12.12 \times 10^{-3}$ \\
\hline 0.1 & 4000 & 2.719 & $-4.98 \times 10^{-3}$ & 22.24 & 2.807 & $11.54 \times 10^{-3}$ & 2.807 & $11.33 \times 10^{-3}$ \\
\hline 0.1 & $<1000$ & 2.721 & $-4.54 \times 10^{-3}$ & 22.28 & 2.809 & $12.82 \times 10^{-3}$ & 2.809 & $12.17 \times 10^{-3}$ \\
\hline
\end{tabular}

*95\% Confidence Level

Table 6. Fits of the data to various models of distortions of the CBR spectrum. 

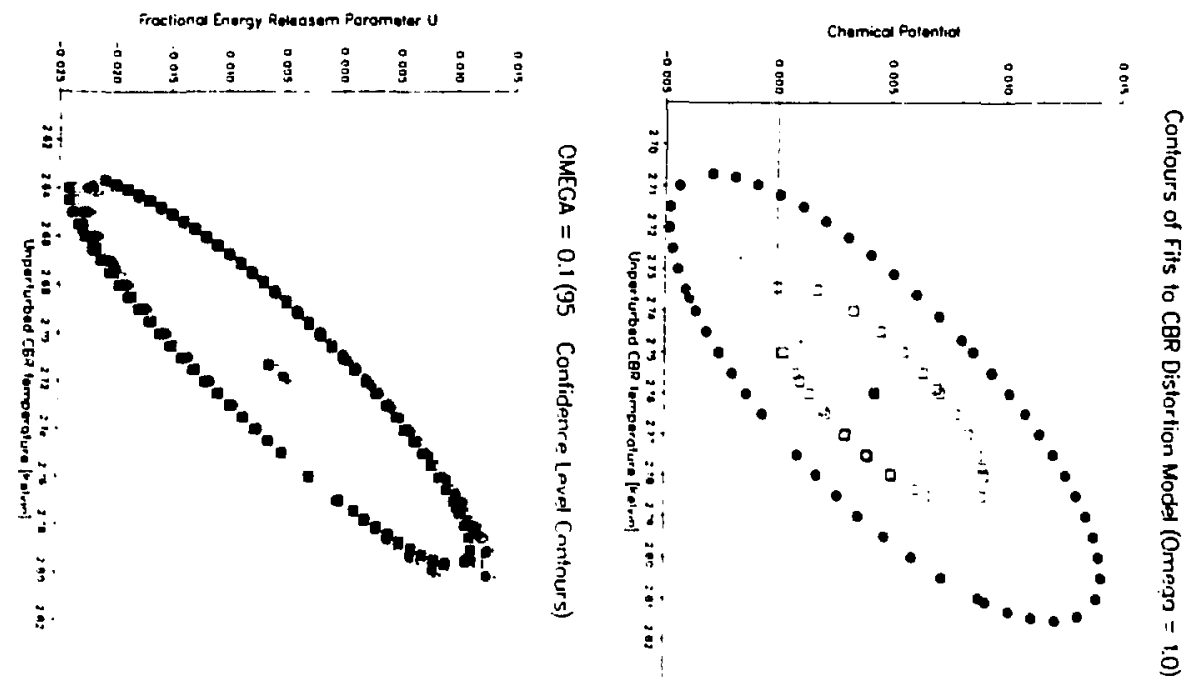

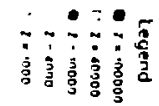

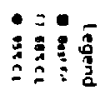
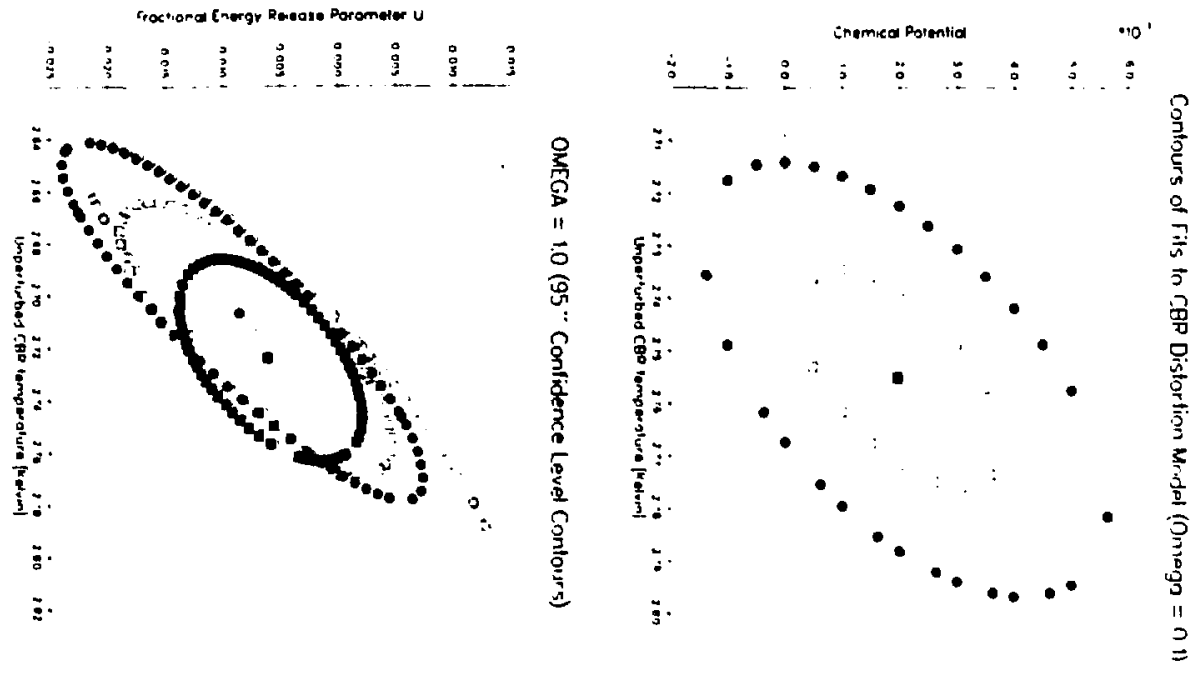

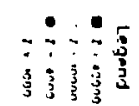

:ִ:

Figure 23. Contour plots of constant $\chi^{2}$ for the CBR spectral distortion models discussed in the text. 
95\% Confidence Limits on Fractional Energy Release

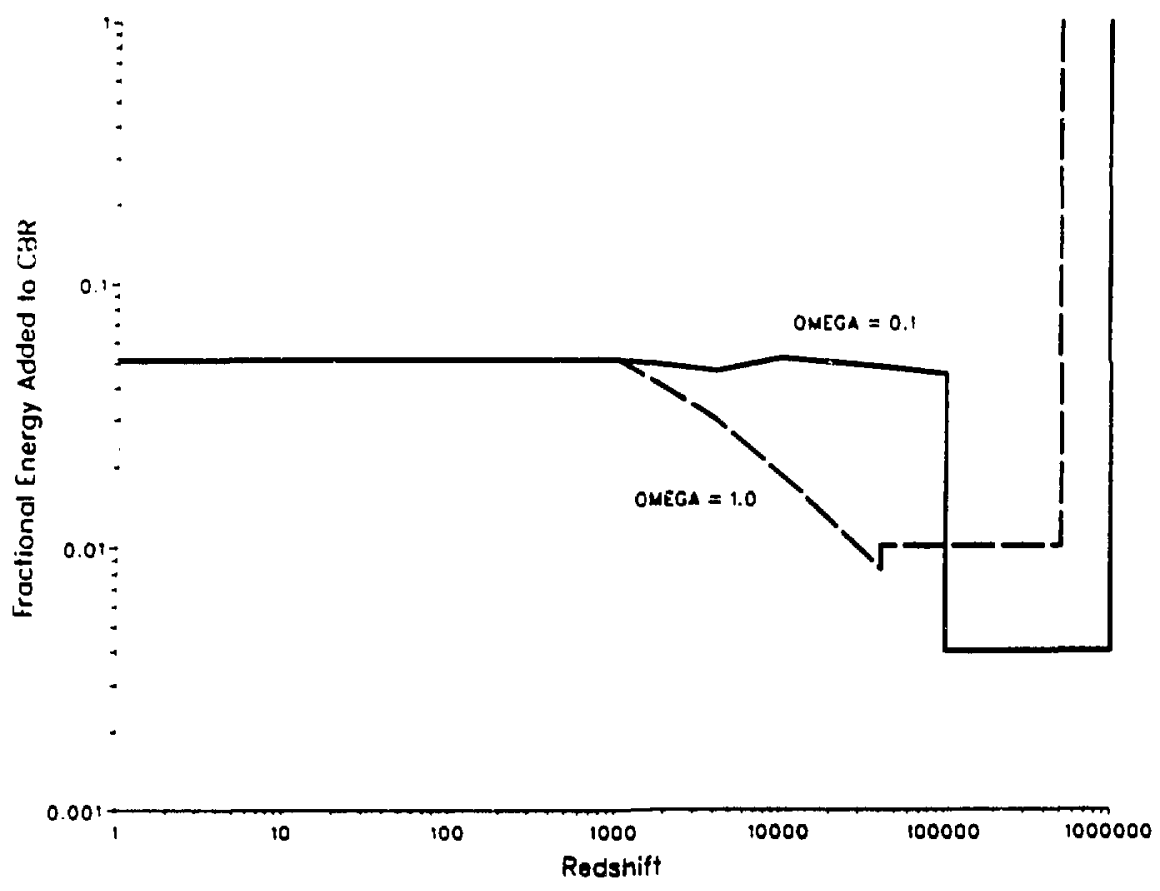

Figure 24. Limits on fractional energy release to the CBR derived from measurements of the CBR spectrum. The limits shown are for energy releases as described in the text. In particular, note that energy releases tu t. matter after $z \approx 1000$ may not be transferred to the CBR. 
maximum values for the distortion parameters under various assumptions about the density of the universe and the timing of energy releases. The best undistorted fit is $T=T_{0}=2.741 \pm 0.016 \mathrm{~K}$ with a $\chi^{2}$ of 22.54 , and the best fitting, "'on-negative distortion is $\mu_{0}$ $=0.00197$ with a $\chi^{2}$ of 21.22. The undistorted fit has 17 degrees of freedom, and because of the additional parameter, the distorted fit has 16 degrees of freedom, so the improvement in $\chi^{2}$ is not statistically significant, and the data are still consistent with a purely Planckian spjectrum.

Having found no distortion in the spectrum, we can use the absence of a distortion to place limits on energy releases to the CBR. Figure 24 shows limits on fractional energy release to the $C B R$ at various times for assumed density parameters of $\Omega_{b}=0.1$ and $\Omega_{b}=1.0$. The limits are based on the contours shown in Figure 23, and must be regarded with the caveat that they refer only to energy releases as described in the text.

Within the last month, Lange et al. (1987) have made new measurements of the CBR at 303,411,645, 1034, 2400, and 3158 GHz. Although the results have not been published as of April 1987, preliminary data are given in Table 7. Measurements at the highest three frequencies are thought to be dominated by interstellar dust, and therefore are not indicative of the CBR. The preliminary results at the lower three frequencies, however, indicate an apparent 


$\begin{array}{ccr}\begin{array}{c}\text { Frequency } \\ \text { (GHz) }\end{array} & \begin{array}{c}\text { Bandwidth } \\ \text { (approximate) }\end{array} & \begin{array}{r}\text { TCBR (Kelvin) } \\ \text { (preliminary) }\end{array} \\ 3158 & 853 & 9.48 \pm 0.11 \\ 2400 & 1128 & 8.05 \pm 0.14 \\ 1034 & 517 & 3.90 \pm 0.07 \\ 645 & 155 & 2.98 \pm 0.03 \\ 411 & 144 & 2.90 \pm 0.03 \\ 303 & 94 & 2.78 \pm 0.03\end{array}$

Table 7. Preliminary data from Lange et al., 1987 


\begin{tabular}{|c|c|c|c|c|c|c|c|c|}
\hline \multirow{2}{*}{\multicolumn{2}{|c|}{$\begin{array}{l}\Omega_{b} \quad z_{h} \\
\text { Larges }\end{array}$}} & & Best & Fit & \multicolumn{3}{|c|}{ Largest $\mu_{0} *$} & \\
\hline & & $\begin{array}{l}\mathbf{T}_{0} \\
\mathbf{T}_{\mathbf{0}}\end{array}$ & $\mu_{0}$ & $x^{2}$ & $\mathbf{T}_{0}$ & $\mu_{0}$ & $\mathbf{T}_{\mathbf{0}}$ & $\mu_{0}$ \\
\hline & $>40000$ & 2.841 & $15.84 \times 10^{-3}$ & 64.52 & 2.855 & $23.50 \times 10^{-3}$ & 2.868 & $21.46 \times 10^{-3}$ \\
\hline & $>100000$ & 2.824 & $5.61 \times 10^{-3}$ & 70.68 & 2.835 & $9.06 \times 10^{-3}$ & 2.849 & $7.01 \times 10^{-3}$ \\
\hline
\end{tabular}

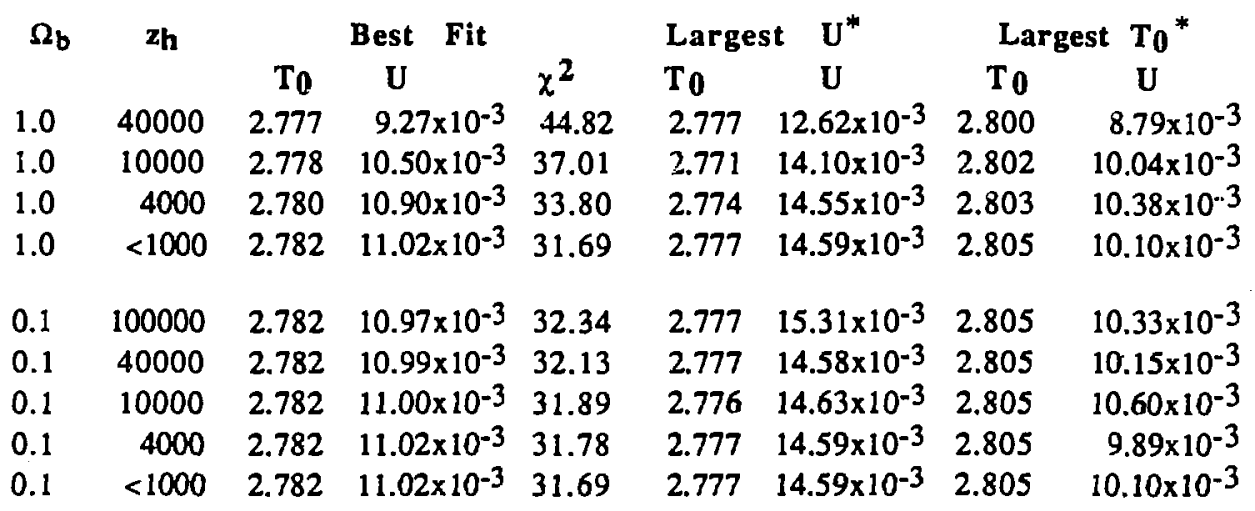

*95\% Confidence Leve!

Table 8. Fits of the data to various models of distortions of the CBR spectrum, using the preliminary data from Lange et al, as well as the other recent measurements. 
distortion in the spectrum of the CBR.

Table 8 shows the best fit and 95\% confidence level values for the distortion parameters using the new data in conjunction with the data in Table 7. The increase in $\chi^{2}$ when the new data is added is due to the conflict between the measurements by Lange et al. and the earlier result by Peterson, Richards, and Timusk. With the new data, the best fit has a $\chi^{2}$ considerably lower than the best fit to an undistorted blackbody. The data are fit nearly equally well by distortion models with distortion parameter $u$ of 0.011 and redshift $\mathrm{z}_{\mathrm{h}}$ ranging from a few to a few times $10^{4}$. Sample plots of the model spectra are shown in Figure 25.

Another possible distortion model is based on the idea that much of the CBR comes from intergalactic dust grains (Hawkins \& Wright, 1987). With such a model, the dust grains must be elongated, to provide a non-Planckian spectrum which resembles those plotted in Figure 26. In this model there is a downturn in the spectrum at frequencies below several $\mathrm{GHz}$, depending on the shape of the dust grains. If the Lange et al. result does indeed indicate a nonPlanckian spectrum, further measurements at $1.4 \mathrm{GHz}$ would be helpful in determining the nature of the distortion. 

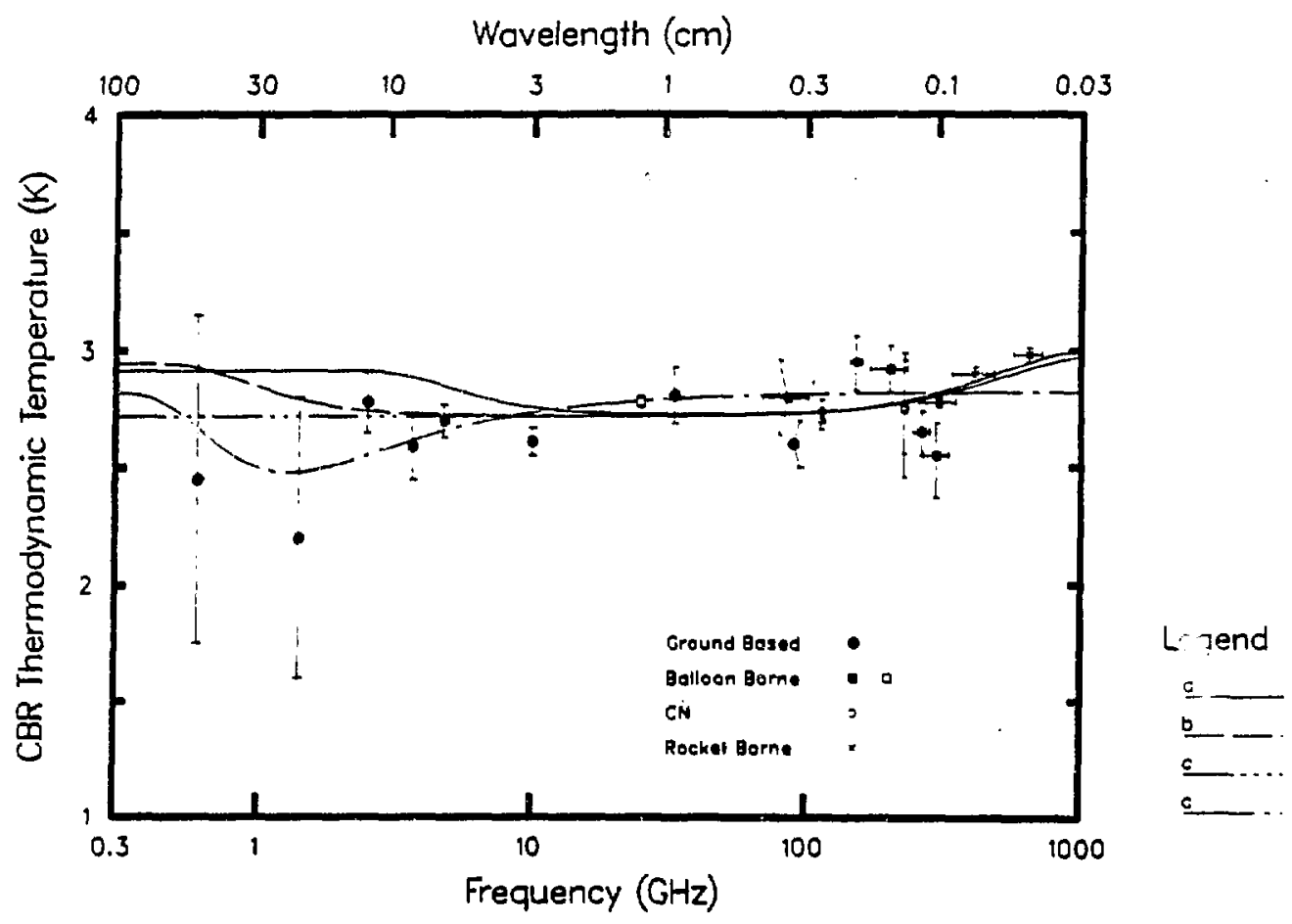

Figure 25. Sample CBR spectral distortions superimposed on existing measurements of $T_{C B R}$. Distortions shown are:

a) $\quad \Omega_{b}=1.0, \quad U=9.27 \times 10^{-3}, \quad z_{h}=40000, \quad T_{0}=2.777$.

b) $\quad \Omega_{\mathrm{b}}=0.1, \quad \mathrm{U}=10.97 \times 10^{-3}, \mathrm{z}_{\mathrm{h}}=100000, \mathrm{~T}_{0}=2.782$.

c) $\quad \Omega_{\mathrm{b}}=0.1, \quad \mathrm{U}=9.27 \times 10^{-3}, \quad \mathrm{z}_{\mathrm{h}}<1000, \quad \mathrm{~T}_{0}=2.824$.

d) $\Omega_{\mathrm{b}}=0.1, \mu_{0}=5.61 \times 10^{-3}, \quad \mathrm{z}_{\mathrm{h}}>100000, \mathrm{~T}_{0}=2.824$. 


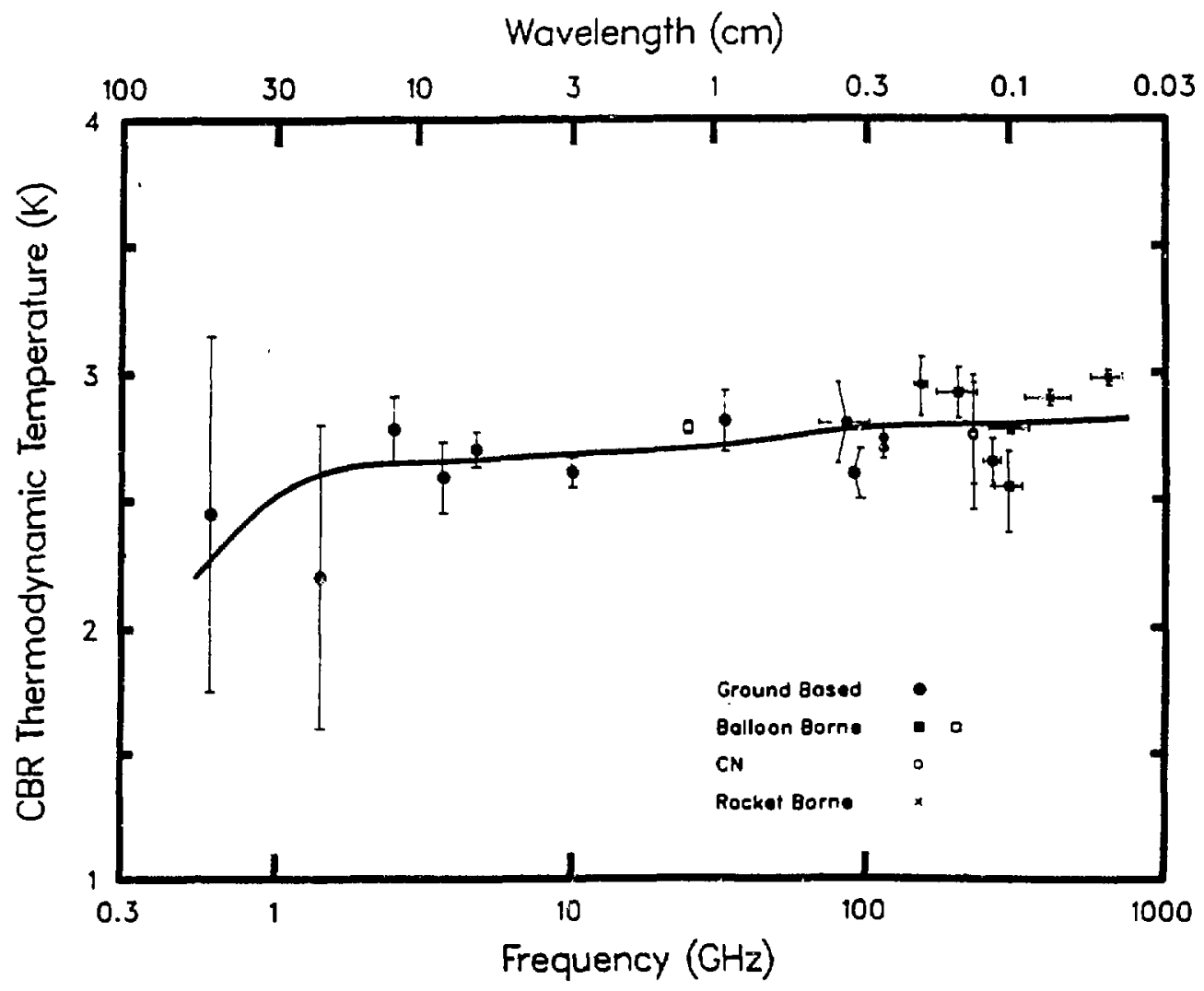

Figure 26. Sample spectrum of CBR derived from a dust model (Hawkins and Wright, 1987). There are a number of free parameters in the model, and the predicted spectrum can vary substantially. 
There are a number of improvements possible in the $1.4 \mathrm{GHz}$ radiometer, and, provided funding is available, we intend to improve the instrument and repeat the measurement. The dominant sources of error are reflections from the LHe target, and uncertainty in the galactic contribution. Both of these can be improved.

The most obvious way to reduce the error caused by reflections from the LHe target is to measure the reflections from the target in situ, under conditions as similar as possible to the actual measurement. After the weather clears, we intend to return to Barcroft and use the same techniques we used to measure the radiometer response to reflections from the simulated dewar (see Section ii of Chapter V) on the actual LHe dewar. This should allow us to determine the phase of reflections from the dewar, and reduce the $0.52 \mathrm{~K}$ uncertainty in $T_{A, l o a d}$ by a factor of 2 or more, depending on what the phase happens to be.

We can go even further, provided we get funding to continue the project, by repeating the entire measurement after replacing the first amplifier in the radiometer with a High Electron Mobility Transistor (HEMT) amplifier. Such amplifiers have been built recently in this frequency range with noise temperatures as low as $30 \mathrm{~K}$ when operating at room temperature and as low as $4 \mathrm{~K}$ when cooled with liquid nitrogen. Because the antenna temperature of the power broadcast by the radiometer is generally the same as the system temperature, this could potentially make reflection effects negligible. 
Reducing the system temperature could also improve the measurement of the galactic signal. While we should be able to reduce the uncertainty in $T_{\text {galaxy }}$ by simply doing multiple galactic scans and averaging the results, a lower system temperature would mean smaller fluctuations, and the number of galactic scans required could drop from dozens to 1 or 2 . Furthermore, if the system temperature were low enough to eliminate reflections as a problem, we could make measurements of the galaxy directly, by comparing the signal at widely separated frequencies (such as $1.300 \mathrm{GHz}$ and $1.465 \mathrm{GHz}$, the frequencies used for previous galactic scans) using the LHe target for comparison. In effect, by doing the CBR measurement itself at 1.300 and $1.465 \mathrm{GHz}$, we could use the frequency dependence of the galaxy to determine its contribution. The remaining uncertainty in $T_{\text {galaxy }}$ could be as low as $25 \mathrm{mK}$, and would be caused by uncertainty in the galactic frequency dependence.

Similarly, the uncertainty in gravitational stress effects from inverting the radiometer could also be reduced, by improving the target used for flip tests, and by performing more such tests with different targets. Uncertainty in the actual measured signal difference between target and sky, $G\left(S_{\text {zenith }}-S_{\text {load }}\right)$, might also be reduced, by studying and reducing the effects of microphonics.

In general, substantial improvements could be made in many areas of radiometer design and technique. This first use of the instrument has provided useiui scientific information, but it has also taught us 
about the experiment itself. With the benefit of experience in its operation, we should be able to substantially improve the radiometer's performance. 
It is impossible to list all of the people who have helped make this dissertation possible, but $I$ will at least attempt to thank a representative sample.

I thank George Smoot, for guidance, motivation, assistance, and tolerance. Giovanni De Amici has been extremely helpful as a colleague, and even more helpful as a friend. Chris Witebsky has provided advice and support continually from the time I first joined the research group through the present. Scott Friedman helped me get started on research when I first arrived at LBL.

The $1.4 \mathrm{GHz}$ radiometer could never have been built without Marc Bensadoun, and $\mathrm{Al} \mathrm{Kogut} \mathrm{and} \mathrm{Marco} \mathrm{Bersanelli} \mathrm{contributed} \mathrm{greatly} \mathrm{to}$ the project. Carol Stanton and Jon Aymon each added contributions to the project.

I kept John Gibson busy with last-minute electronics problems right up until the last moment, and without his expertise, patience, and talent none of the electronics would ever have worked.

The entire staff of the White Mountain Research Station have invariably been helpful, tolerant, and understanding. In particular, the Barcroft crew have always made the extra effort to ensure that this project (and every project) was a success. The White Mountain Research Station also provided financial support at a critical juncture.

Phil Lubin, Terry Mast, Rich Muller, Giorgio Sironi, and Luis Alvarez have all contributed greatly to my education, and I have benefitted 
from associating with each of them. Faye Mitschang, Nancy Gusack, and Janice Gates have all helped at various times by guiding me through the sea of paper that keeps major institutions like LBL, Space Sciences Laboratory, and the University of California afloat.

I particularly want to thank Dave Williams and Tap Lum at the Radio Astronomy Lab, for invaluable advice and assistance, and for coming through with a replacement amplifier when all seemed lost.

I'd like to thank Hal Dougherty and Armi Meuti, for teaching me how not to kill myself in the machine shop, and for putting up with the mistakes of yet another novice machinist. Equipment built by $\mathrm{Hal}$ invariably works better, looks better, and lasts longer than our attempts to. emulate his skill.

For long hours above and beyond the call of duty spent on the doomed tunable radiometers, I thank Linda Kelley, who was invaluable as an assistant, colleague, and co-conspirator. I want to thank Bruce Grossan and Mark Griffith for their help and support, and for keeping me sane under insane conditions. I particularly appreciated Bruce's occasional fixes of Star Trek and pseudo-Chinese food.

I thank Nazzareno Mandolesi and CNR for the loan of antennas used in the $2-8 \mathrm{GHz}$ radiometer.

The Lawrence Berkeley Laboratory is an unparalleled place to do scientific research, and the wealth of facilities and expertise available 
Page $96 \quad$ Acknowledgements

here have made it a superb place to learn physics. This work has been supported by National Science Foundation grant number AST 8406187 and the Department of Energy under contract number DEAC03-76SF00098.

Special thanks to my sister, Sandi Levin, who not only provided the support I have come to expect from her, but also undertook the monumental task of editing this manuscript.

Last, but by no means least, I thank my parents, Larry Levin and Sylvia Levin, for their constant support and encouragement throughout my education, and for teaching me at an early age to ask questions and look for answers. It was a special joy to work with my father briefly at Barcroft, and both my parents have always been there when I needed them. 
Bielli, P., Pagana, E., and Sironi, G., 1983, Proceedings ICAP, 1, 509.

Berkhuijsen, E. M., 1972, Astron. Astrophys. Suppl. 5, 263

Chan, K. L., and Jones, B. J. T., 1975, Ap. J., 195, 1.

Chan, K. L., and Jones, B. J. T., 1976, Ap. J., $200,461$.

Costales, J.,1984, A.B. Thesis, Lawrence Berkeley Laboratory, 18744.

Costales, J., et al., 1986, Radio Science, 21, 47.

Crane, P., Heygi, D. J., Mandolesi, N., and Danks, A. C., 1986, Ap. J., 309,822

Crane, R., 1981, Proc. IEEE, 69(2), 196.

Danese, L., and De Zotti, G., 1977, Riv. Nuovo Cimento, 7, 277.

Danese, L., and De Zotti, G., 1978, Astron. Astroph., 68, 157.

Danese, L., and De Zotti, G., 1980, Astron. Astroph., 84, 364.

Danese, L., and De Zotti, G., 1982, Astron. Astroph., 107, 39.

Danese, L., 1984, Padua Memo 2, unpublished.

De Amici, G., Smoot., G., Friedman, S.D., and Witebsky, C., 1985, Ap. J., $298,710$.

De Amici, G., Smoot, G., Aymon, J., Bersanelli, M., Kogut, A., Levin, S., and Witebsky, C., 1987 In preparation for submission to Ap.J.

de Vaucoleurs, G., 1979, Ap.J., 233, 433

Donnelly, R. J.,1967, Experimental Superfluidity, University of Chicago Press, Chicago Il.

Haslam, C.G.T., Salter, C.J., Stoffel, H., and Wilson, W.E., 1982, Astr. Astroph. Suppl. Ser., 47, 1. 
Hawkins, I., and Wright, E. L., 1987 In preparation

Illarionov, A. F., and Sunyaev, R. A. 1974, Astron. Zh., 51, 1162 (1975, Soviet Astron., 18, 691).

Johnson, D. G., and Wilkinson, D. T., 1986, Ap. J. (Letiers), 313, L1-L4.

Jones, B. J. T., 1980, Physica Scripta, 21, 732.

Kogut, A., et al., 1987, in preparation for submission to Ap. J.

Kraus, J. D., 1966, Radio Astronomy, 241-244, McGraw-Hill, New York

Lange, A., personal communication, April 1987

Liebe, H.J., 1981, Radio Sci., 16(6), 1183.

Lightman, A. P., 1981, Ap. J., 244, 392.

Mandolesi, N., Calzolari, P., Cortiglioni, S., and Morigi, G., 1984, Phys. Rev. D, 29, 2680.

Mandolesi et al., 1986, Ap. J., 310, 561.

Meyer, D.M., and Jura, M., 1984, Ap. J. (Lett.), 276, L1.

Meyer, D.M., and Jura, M., 1985, Ap. J., 297, 119.

Otoshi, T.Y., and Stelzried, C.T., 1975, IEEE Trans. Instr. Meas., 24, 174.

Penzias, A.A., and Wilson, R.W., 1965, Ap. J., 142, 419.

Peterson, J.B., Richards, P.L., and Timusk, T., 1985, Phys. Rev. Lett., $55,332$.

Ramo, S., Whinnery, J. R., Van Duzer, T., 1965, Fields and Waves in Communication Electronics, 589-611, Wiley \& Sons, Inc., New York

Reich, W., 1982, Astron. Astrophys. Suppl. 48, 219 
Rosenkranz, P. W., 1975, IEEE Trans. Antennas Propagation, AP-23(4), 498

Sandage, A.R., Tammann, G., 1976, Ap. J., 210, 7

Sironi, G., and Bonelli, G.,1987, Ap. J., in press.

Smoot, G., et al., 1983, Phys. Rev. Letters, 51, 1099

Smoot, G., et al., 1985a, Conference Proceedings, Societa' Italiana di Fisica, 1, 27.

Smoot, G., et al., 1985b, Ap. J. Lett., 291, L23.

Smoot, G., 1986, Highlights of Astronomy, Reidel IAU Conference Proceedings, 7, 297-305.

Smoot, G., Levin, S., De Amici, G., and Witebsky, C., 1987, Radio Science, in publication.

Smoot et al., 1987, Ap. J. (Lett.), submitted 1987.

Sunyaev, R. A., and Zel'dovich, Ya. B., 1969, Astrophys. Space Sci., 4, 301.

Sunyaev, R. A., and Zel'dovich, Ya. B., 1959, Astrophys. Space Sci., 7, 20.

Sunyaev, R. A., and Zel'dovich, Ya. E., 1980, Ann. Rev. Ast. Ap., 18, 537.

Ulaby, F.T., Moore, R.K., and Fung, A.K., 1981, Microwave Remote Sensing: Active and Passive, Vol. 1, 256-343, Addison-Wesley, New York.

Waters, J. R., 1576, "Absorption and Emission by Atmospheric Gases," in Methods of Experimental Physics, vol. 12B, Ed. M. L. Meeks, Academic Press, NY.

Webster, A. S., 1974, Mon. Not. Royal. Astron. Soc., 166, 355 
Weiss, R., 1980, Ann. Rev. Ast. Ap., 18, 489.

Witebsky, C., Smoot, G., De Amici, G., and Friedman, S.D., 1986, Ap. J., $310,145$.

Witebsky, C., Smoot, G. F., Levin, S., ànd Bensadoun, M., 1987, submitted to IEEE Antennas and Propagation (reprinted here as Appendix C)

Wright, E. L., 1982, Ap. J., 255, 401. 
It is a truism that we learn from our failures at least as much as from our successes, and the $2-18 \mathrm{GHz}$ radiometers are an example of that fact. While they were not successful as instruments to study the spectrum of the CBR, they were (and are) useful for other purposes, including the purpose of learning from previous mistakes.

Originally the $2-18 \mathrm{GHz}$ radiometers were to be a single instrument, tunable from 2 to $18 \mathrm{GHz}$, and capable of measuring the CBR antenna temperature to an absolute precision of $0.10 \mathrm{~K}$. This tunable radiometer consisted of two Dicke-switched differential radiometers sharing the same pair of antennas. (see Figure A1), using opposite linear polarization states. I will describe the operation of the 7.5-18 $\mathrm{GHz}$ radiometer, but the $2-8 \mathrm{GHz}$ radiometer was very similar.

Immediately following the antennas, signals passed through a PIN diode switch to the first amplifier, so that the radiometer alternately viewed each antenna. The signal was then amplified by a GaAs FET amplifier, sent through a YIG filter, amplified again, and sent to the detector diode. The output of the detector diode went to a lockin amplifier/integrator. The lockin amplifier and integrator synchronously amplified that portion of the signal which switched at the $100 \mathrm{~Hz}$ operating frequency of the Dicke switch. Thus the radiometer output was proportional to the difference in power received at the two antennas. The YIG filter was controlled by a computer via a digital to analog converter (DAC), and normal operating procedure was to linearly sweep the frequency from 7.5 to 
Broadband

Antennas

(Dual Polarization)
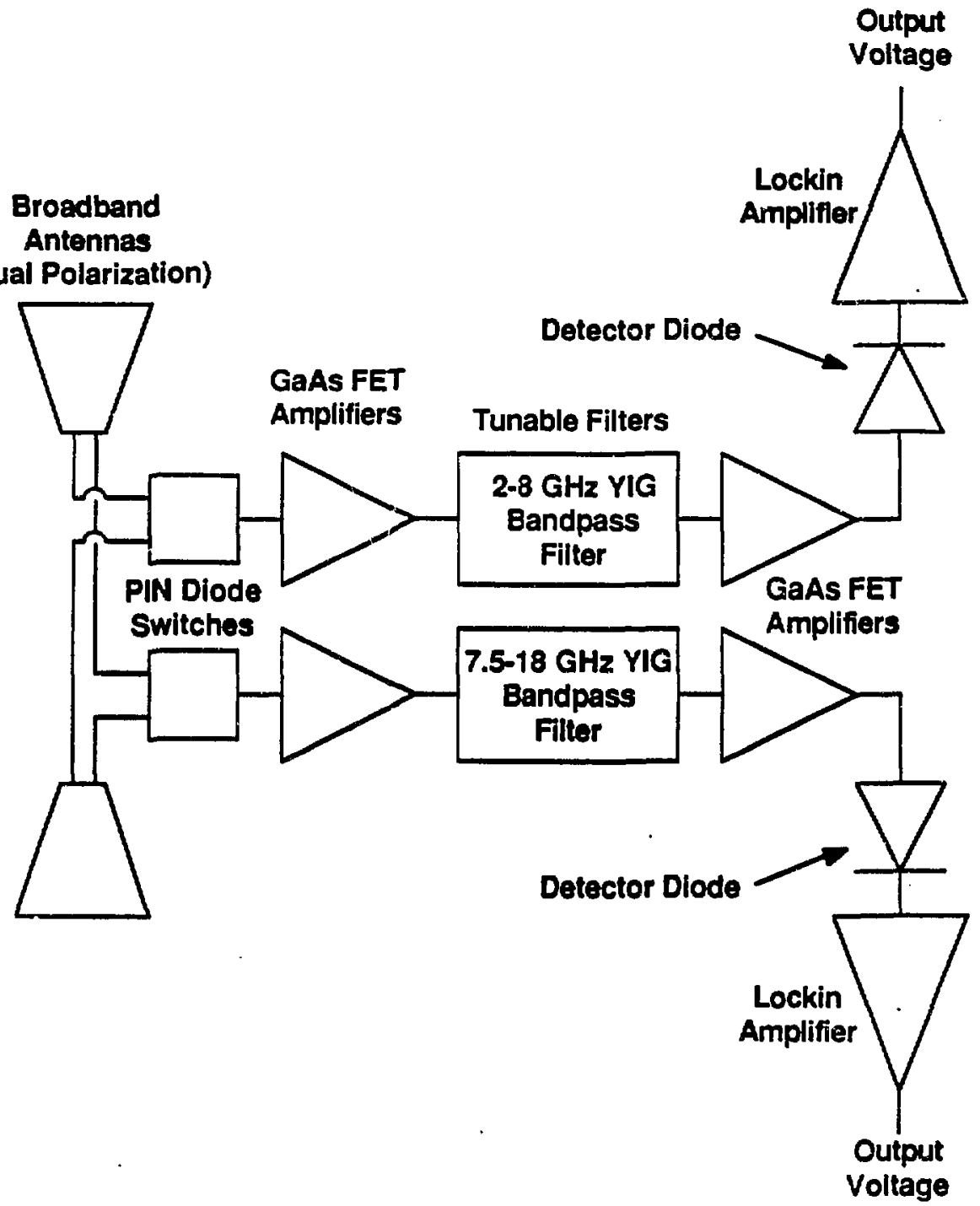

Figure Al. Schematic of the 2-18 GHz radiometers. 
18.0 GHz in 256 steps, each lasting 1 second. The YIG bandwidth was $200 \mathrm{MHz}$, so this constituted a substantial oversampling. The radiometer output went through an Analog to Digital Converter (ADC) to the same computer which controlled the YIG, for storage on floppy disk and for real-time analysis.

The $2-8 \mathrm{GHz}$ radiometer was essentially the same as the $7.5-18 \mathrm{GHz}$ radiometer, and the switching was phased such that the radiometers alternated antennas, with the $2-8 \mathrm{GHz}$ radiometer looking out antenna $A$ whenever the $7.5-18 \mathrm{GHz}$ radiometer was looking out antenna B. By having the two radiometers use opposite antennas at any one time, we eliminated the possibility of cross-talk between the two radiometers.

The 2-18 GHz radiometer is shown in Figure A2. The radiometer was mounted on bearings, so that it could be rotated to have each antenna face vertically or horizontally, while the other antenna faced the opposite direction. The radiometer was on a cart capable of being rolled along the rails shown in Figure 6, and movable reflectors were mounted on the cart to make atmospheric scans possible. As shown in Figure A2, each of the two reflectors could be tipped to either 45 or 25 degrees, so that the radiometer viewed (in reflection) either the zenith sky or an angle 40 degrees from zenith. The reflectors and the cart were surrounded with reflecting "ground 


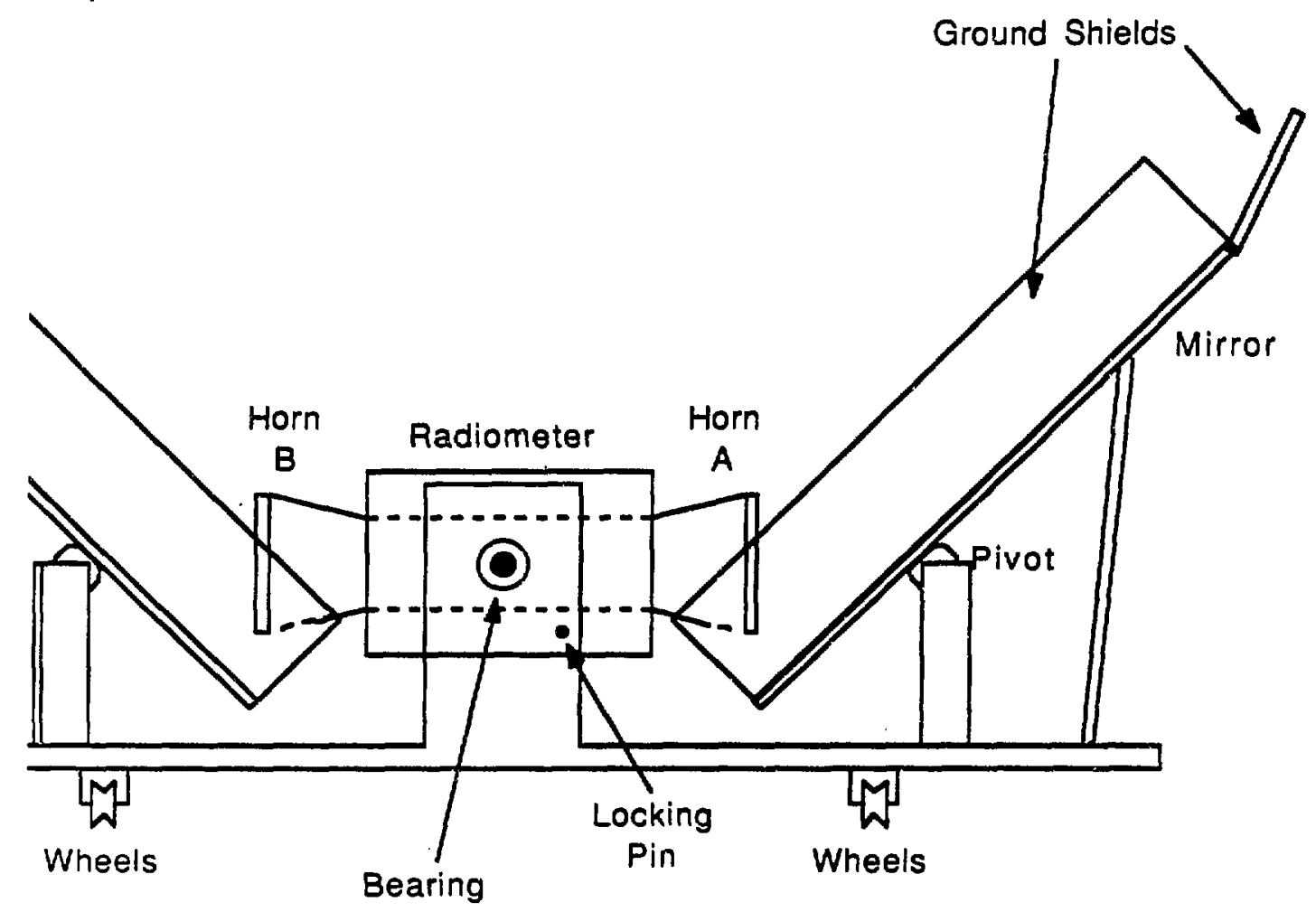

Figure A2. The 2-18 $\mathrm{GHz}$ radiometer. 
Appendix A: The 2-18 $\mathrm{GHz}$ Radiometers Page A5

shields" to redirect to the sky any part of the antenna pattern which spilled over the reflectors.

Tests in Berkeley soon showed that additional shielding was needed when the radiometer was oriented to view the vertical sky. As shown in Figure A3, we built a large conical ground shield, along with a mechanism for raising and lowering it to allow the radiometer to rotate.

Preliminary work in 1982 and 1983 revealed problems with RF interference, and we replaced the 2-pole YIG filter in the $2-8 \mathrm{GHz}$ radiometer with a 4-pole YIG filter with a $50 \mathrm{MHz}$ bandwidth, in order to reduce the effect of out-of-band interference.

In 1984 we brought the $2-18 \mathrm{GHz}$ radiometer to the remote site on Mount Barcroft, and, while attempting to make CBR measurements, discovered a number of problems. From then until July of 1986, I spent most of my time isolating and trying to solve those problems.

Because of the large bandwiath requireu, ine $2-18$ GHz antennas did not have low enough sidelobe response, particularly at low frequencies, to sufficiently reduce the ground contribution. We attempted to solve this problem by building big ( $3 \times 5$ meters) ground screens at the Barcroft observing site (the screens were too large to transport or to use at our test facility in Berkeley), but found 


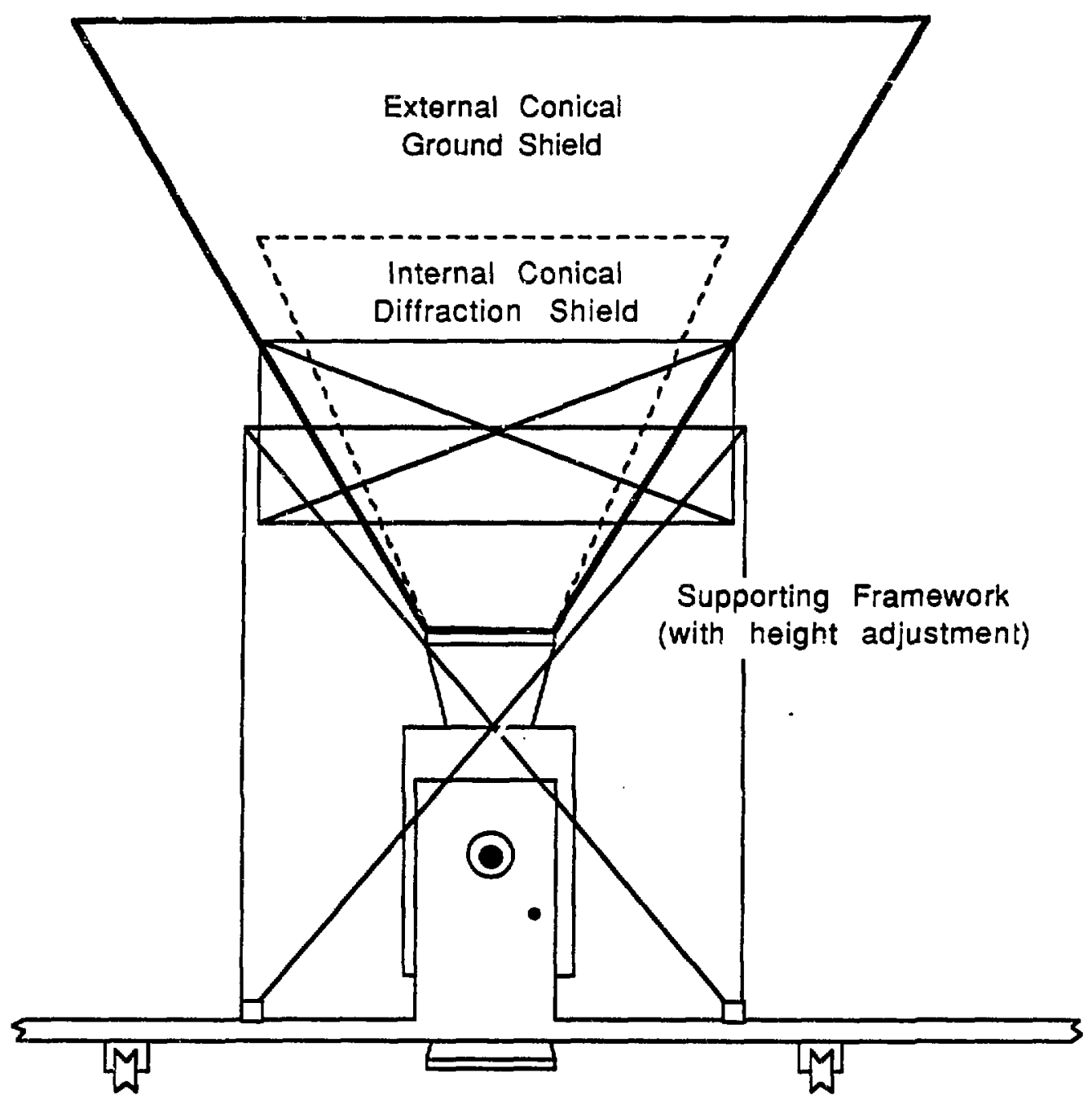

Figure A3. The 2-18 GHz radiometer with conical ground shield. The ground shield was raised several $\mathrm{cm}$ when the radiometer was rotated, and was then lowered back into position. 
the screens less effective than desired, especially at the lowest frequencies.

The major drawback to our frequency-scanning pattern with the YIG filters was that it took nearly 5 minutes to run through a single set of frequencies. Thus there was a minimum delay of 5 minutes between viewing any two targets. Thus temperature instability, and its attendant drifts in radiometer gain, was an important problem. Because the ambient temperature at the remote observing site is considerably lower than in Berkeley, it was not possible to optimize the radiometer's thermal characteristics for maximum control both in Berkeley and at the observing site. In 1984 and earlier, we had settled for a system which would not thermally regulate in Berkeley, but was thermally controlled by heaters when we were at the White Mountain research station. In 1984 we became convinced that the radiometer's temperature needed to be held more constant, and in 1985 we arranged removable thermal insulation, and used an air conditioner to artificially cool the radiometers, in order to partially simulate the White Mountain temperatures in Berkeley. In this fashion we were able to experiment with and improve the placement of the heaters and the thermal regulation settings to make the radiometers' gain more stable.

The most important problem discovered in 1984 was that the radiometer was seeing its own reflection from virtually every target. 
The 2-8 $\mathrm{GHz}$ radiometer and the $7.5-18 \mathrm{GHz}$ radiometer each broadcast out the antenna power equivalent to approximately $300 \mathrm{~K}$. There were frequency-dependent reflection effects from the conical ground shield, the interface between the radiometer and the LHe dewar, and the reflectors used for atmospheric scans, at levels ranging from nearly 0 to approximately $1 \mathrm{~K}$, depending on frequency and target.

As an attempt to solve these problems, in 1985 we split the $2-18 \mathrm{GHz}$ instrument into two separate radiometers, with two sets of antennas. The 7.5-18 GHz radiometer used the same antennas as before, but the 2-8 GHz radiometer was put in an entirely new housing, using antennas originally designed to operate at $4.75 \mathrm{GHz}$ (borrowed from Nazzareno Mandolesi at Istituto TESRE-CNR, Bologna, Italy). In this fashion, we could re-design the ground shields and dewar interface to be more effective within their respective (narrower) bandwidths.

To provide a better check on reflections, we added a directional coupler and a noise source to each radiometer, as shown in Figure A4. When the noise source was on, the power broadcast by the radiometer was increased by nearly an order of magnitude, so reflection effects became easily visible. We soon realized that coherent interference effects (see Chapter $V$, Section ii) made the reflection effects still difficult to measure precisely, but with the noise source arrangement we were able to conveniently determine the magnitude of the problem for each target viewed. 
Appendix A: The 2-18 GHz Radiometers Page A9

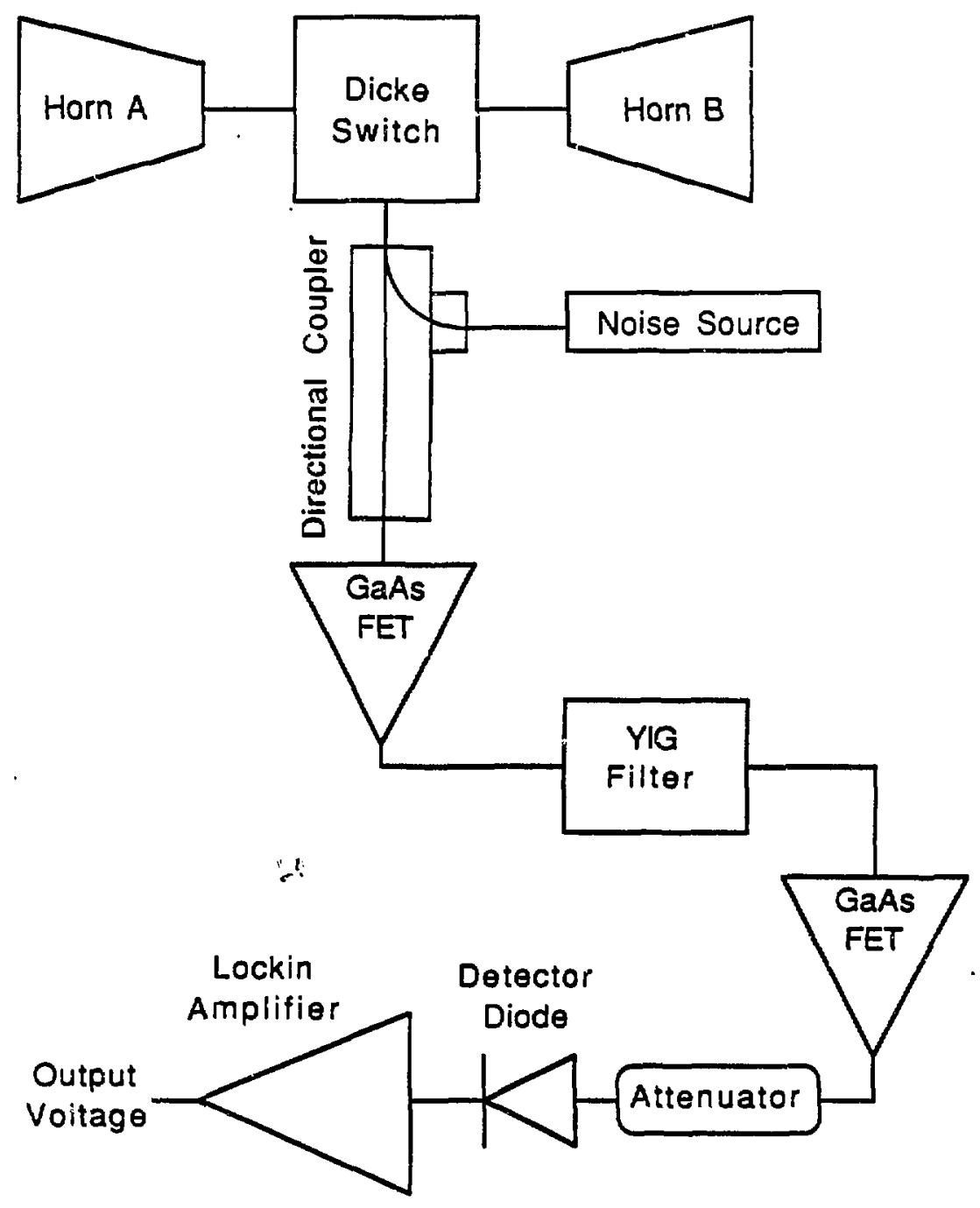

Figure A4. Schematic diagram of the $2-8 \mathrm{GHz}$ and $7.5-18 \mathrm{GHz}$ radiometers in 1986. 
In order to use the $4.75 \mathrm{GHz}$ antennas over a bandwidth from 2 to 8 $\mathrm{GHz}$, we needed to build an adaptor and modify the horn throat. We originally contracted with Passive Microwave Technology in Canoga Park, California to build a pair of low-reflection adaptors from the antennas to coaxial cable, but (nearly a year after the original deadline) they were unable to meet the performance originally specified. We therefore bought a pair of low-reflection SMA/doubleridged-waveguide launchers from Continental Microwave (model DRA250-SMA-F) and built the rest of the adaptors ourselves, modifying the throat of the antenna by trial and error with aluminum foil until we had achieved a satisfactory design, which we machined out of aluminum and bolted inside the antennas.

In order to make the new antennas and adaptors work, we had to accept a slightly reduced bandwidth, so the radiometer was only effective from 2.5 to $7.5 \mathrm{GHz}$. The frequency-dependent reflection from the antennas and adaptors was still as high as several per cent at some frequencies within this reduced range, but they were quite stajle, and the differential character of the measurement makes reflection effects dependent only on the change in reflection when the radiometer views different targets.

We constructed a new cart and housing for the 2-8 GHz radiometer (see Figure A5), and mounted the $2-8 \mathrm{GHz}$ components inside the 


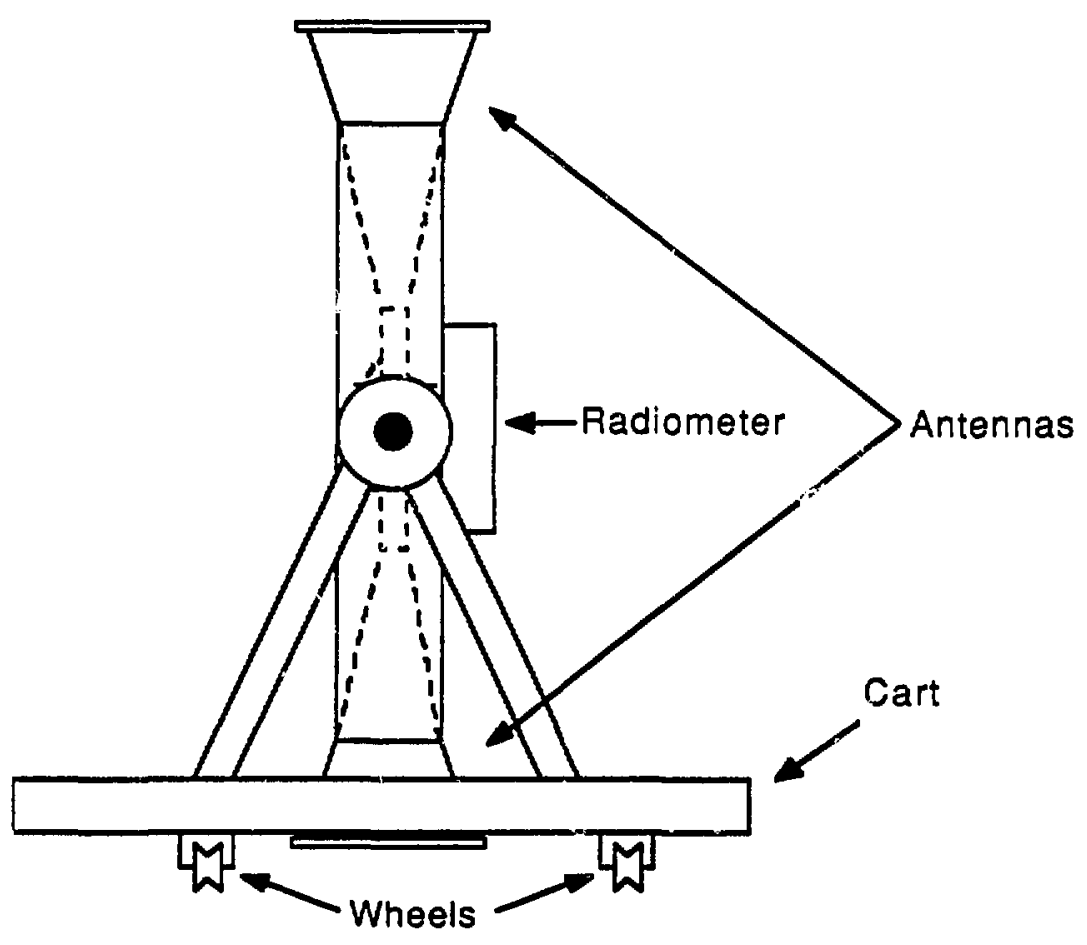

Figure A5. The 2-8 $\mathrm{GH}_{2}$ radiometer and cart in 1986. 
new housing. As discussed above, the electronic components were thermally regulated, and we rearranged the heaters and thermal sensors to improve thermal stability. The dominant source of thermal variation in each radiometer was the YIG filter, which produces waste heat dependent on the current driving the coils, and hence dependent on the frequency setting. We arranged the temperature sensors and heaters in each of the two radiometers to respond more quickly to sudden changes in the YIG temperature, and we lowered the regulated temperature of the radiometer as a whole, to allow the YIG's internal temperature regulation system to become more effective.

The 2.5-7.5 GHz bardwidth of the new low-frequency instrument was still too broad to allow us to use corrugated antennas, so the sidelobes on the $2-8 \mathrm{GHz}$ radiometer were substantial. We built a large structure of screens and aluminum sheet to serve as ground shields, and used measurements of the antenna gain pattern (with screens attached) to determine the contribution from the ground. After considerable effort, we achieved a design which reduced the ground contribution to an acceptable level when the radiometer was viewing the zenith sky.

Using the noise sources and directional couplers, we had re-designed the ground shields, reflectors, and LHe dewar interface for the 7.5-18 $\mathrm{GHz}$ radiometer so that reflections were no longer significant. For the 
2-8 $\mathrm{GHz}$ radiometer we were able to reduce reflections from the interface and the ground screens to an acceptable level, but were unable to devise a scheme for measuring the atmosphere which had low enough reflection, reduced the sidelobe contribution to acceptable levels, and used equipment small enough to be practicai under the adverse conditions of our remote observing site. Fortunately, the atmospheric contribution is relatively incepcndent of frequency between 2 and $8 \mathrm{GHz}$ (səe Appenidix B), so wo decided to use atmospheric measurements by the $8.18 \mathrm{GHz}$ radiometer and by the radiometer operating at $3.7 \mathrm{GHz}$ to madei to atmospheric contribution between 2.5 and $7.5 \mathrm{GHz}$.

While the problems dessribed aboye, and the compromises they required, did reduce the radiometers' performance, we were still able to continue the project. Nonetheless, these problems absorbed considerable time and efrort, and by the middle of 1986 the research group was developing new radiometers operating a: 3.7 and $1.4 \mathrm{GHz}$, and we were pressed for resources. Under these conditions, we discovered an additional problem with buth the 2-8 $\mathrm{GHz}$ and $7.5-18$ $\mathrm{GHz}$ radiometers: the PIN diode switches each emit radiation which is dependent on the radiometer's orientation at the 0.2 Kelvin level.

The presence of a 0.2 Kelvin "flip offset" would have been acceptable if the source of the offset were well anderstood and repeatable, so that it could be reliably subtracted. The presence of a yariable flip offset whose cause was not well established, coupled with the other 
Page A14 Appendix A: The 2-18 GHz Radiometers

problems plaguing the $2-18 \mathrm{GHz}$ radiometers, caused us to abandon the 2-18 GHz radiometers in favor of concentrating scarce resources on the new radiometers at $1.4 \mathrm{GHz}$ and $3.7 \mathrm{GHz}$. The $2-18 \mathrm{GHz}$ radiometers have not been used for further CBR measurements.

While the $2-18 \mathrm{GHz}$ radiometers did not provide any useful measurements of the CBR, they did furnish experience in radiometer development. Experience with the 2-18 $\mathrm{GHz}$ radiometers provided the beginning of our current understanding of the coherent reflection problems and the techniques for measuring them. Our experience measuring the gain patterns of the $2-8 \mathrm{GHz}$ antennas and ground shields was useful in measuring the gain patterns of other antennas, and we copied some of the techniques of ground shield construction in connection with other radiometers. In general, building the 2-18 $\mathrm{GHz}$ radiometers was a valuable learning experience. 
In order to measure the brightness temperature of the CBR, we need to determine and subtract the contribution from emission by the atmosphere. In making this determination, we are dependent on a model of the atmosphere. This model has two parts, a spatial dependence and a spectral dependence. For the $1.4 \mathrm{GHz}$ measurement, both aspects of the model are important.

As discussed in Chapter $\mathrm{V}$, Section vi, the atmospheric contribution at 1.4 $\mathrm{GHz}$ was deduced by extrapolating from a measurement at 3.7 GHz. The measurement itself depended on an assumed spatial dependence of the atmospheric emission, and the extrapolation depends on the spectral dependence of atmospheric emission. Putting aside for the moment the actual measurement at $3.7 \mathrm{GHz}$, let us look briefly at the spectral dependence of microwave emission from the atmosphere.

The major contributors to atmospheric emission at $\mathrm{GHz}$ frequencies are oxygen, water vapor, and liquid water. Liquid water contributes primarily through Rayleigh scattering from small droplets, and does not have a significant effect at our $21 \mathrm{~cm}$ wavelength on clear days, especially at the Barcroft site, where the water content is low (Ulaby, 1981, Waters, 1976, Danese, 1984).

The oxygen molecule has no permanent electric dipole moment, but it has a number of magnetic dipole transitions at microwave frequencies. The transition probabilities are small for these lines, but the abundance of oxygen along any line of sight is enough to 
make the oxygen emission significant. The shape and width of the oxygen lines depends on temperature and pressure broadening, but the emission by oxygen is relatively stable at a given site, as the oxygen is well mixed in the atmosphere, and temperature fluctuations are largely balanced by the corresponding density variation (Costales et al. 1985, Smoot et al. 1987a).

Models of water vapor emission are somewhat empirical, since observations do not agree well with theory, but in all models water emission is low at $3.7 \mathrm{GHz}$ and negligible at $1.4 \mathrm{GHz}$, so the error induced is minor (Costales 1984, Liebe 1981, Crane, 1981, Waters, 1976). In this instance we are fortunate that we are extrapolating atmospheric emission between such low frequencies. Water vapor emission is significant at higher frequencies, and measurements of the atmosphere at $3.7 \mathrm{GHz}$ and $10 \mathrm{GHz}$ are only in marginal agreement with the models (De Amici, et al. 1987, Kogut et al. 1987), indicating that an extrapolation from $10 \mathrm{GHz}$ to $1.4 \mathrm{GHz}$ would have been questionable.

The atmospheric measurement at $3.7 \mathrm{GHz}$ is discussed in detail by $\mathrm{De}$ Amici et al. (1987), but I will briefly summarize that discussion here. The simplest approximation to the spatial dependence of atmospheric emission is to treat the atmosphere as an optically thin,. stable, flat slab of uniform temperature, and the antenna's gain pattern as a 2 dimensional delta function. In this oversimplified model, atmospheric emission is simply proportional to the thickness of 
atmosphere viewed at a particular angle, and the atmospheric contribution is

$$
T(\theta)=T(0) / \cos (\theta)
$$

where $\theta$ is the zenith angle of observation. Introducing a layered atmosphere, where temperature and density are functions of altitude, does not change the spatial dependence in this simplified model. We can therefore determine the vertical atmospheric emission by measuring the signal at several zenith angles, and fitting the measurements to Equation B1, under the assumption that there are no other spatially dependent effects. The CBR anisotropy has been measured to be less than $0.1 \%$ on large angular scales, and the Galactic contribution can be measured and subtracted, as discussed in Chapter V, Section iii. The $3.7 \mathrm{GHz}$ radiometer made measurerients at zenith angles of 0,30 , and 40 degrees.

The actual atmosphere is curved, and the antenna has a finite beam width, so Equation B1 must be replaced by a more complicated integral, with some small dependence on the vertical structure of the atmosphere. We used a simple model of the vertical structure, dependent only on a characteristic scale height $(6 \mathrm{Km})$ and a kinetic temperature of the atmosphere $(260 \mathrm{~K})$. Large changes in the parameters produce negligible changes in the calculated atmospheric emission. (A change in scale height of $2 \mathrm{Km}$ or a change in kinetic temperature of $40 \mathrm{~K}$ would result in a change in calculated zenith atmospheric emission of less than $0.001 \mathrm{~K}$.) 
The antenna gain pattern was measured in Berkeley, and numerically integrated to calculate the effect on the apparent spatial dependence of the atmospheric emission. Repeating the analysis with an equivalent gaussian gain pattern, and gaussians wider and narrower by $20 \%$, changed the calculated zenith atmospheric emission by less than $0.02 \mathrm{~K}$.

Additional corrections were made for contributions from the Galaxy and for gain saturation effects. Pointing errors contributed to the error budget, as did possible ground contribution through the antenna sidelobes. The end result was that the zenith atmospheric antenna temperature at $3.7 \mathrm{GHz}$ during the time of $\mathrm{CBR}$ measurements at Barcroft was $0.875 \pm 0.073 \mathrm{~K}$. 


\title{
A LARGE L-BAND RECTANGULAR CORRUGATED HORN
}

C. Witebsky, G. F. Smoot, S. Levin, M. Bensadoun

\author{
Lawrence Berkeley Laboratory \\ and Space Sciences Laboratory \\ University of California, Berkeley
}

\begin{abstract}
This paper describes a lightweight, corrugated-horn antenna, constructed from sheet metal. Over a 1.3-1.7 GHz operating band, ita half-power beamwidth is approximately $20^{\circ}$ in the $E$-plane and varies from $17^{\circ}$ to $13^{\circ}$ in the $H$-plane. Quarter-wave choke slots at the aperture help to reduce the $E$-plane sidelobes below $-55 \mathrm{~dB}$ at angles greater than $90^{\circ}$, while the H-plane sidelobes lie in that range both with and without choke slots. Return loss throughout the operating band is $-25 \mathrm{~dB}$ or below. Critical dimenuions are provided, together with useful guidelines for designing similar antennaa.
\end{abstract}

\section{INTRODUCTION}

We have developed a large (s 2-meter-long), lightweight horn antenna with low sidelobes for measurements of the cosmic background radiation temperature near $1.4 \mathrm{GHz}$. The antenna, a rectangular horn with corrugations on its E-plane walls, is made primarily from aluminum sheet for lightness and ease of fabricaticn. This design has several significant advantages over the conical corrugated horns used at higher frequencies $[1,2 \mid$. I'irst, the horn is much lighter than a machined corrugated horn of comparable sise. Second, the antenna is relatively simple to construct, and does not require any special machining facilities other than a sheet-metal break of sufficient width. Horns of thin sort are especially convenient for prototype development, since characteristics such an the slot width can be readily varied. Third, the horn can be easily broken down into panels for transport. In common with conical corrugated horns, it has low sidelobe levels and a good input match.

This paper describes the design of the antenna and reports the results of our pattern and reflection measurements. 


\section{THEORY}

In contrat to the large body of work published on the theory and design of conical corrugated horns, relatively little has been written about rectangular corrugated horns. Some of the first such horns are described in a paper by Lawrie and Peters [3], who pioneered this Geld. The theory of wave propagation in $E$-plane corrugated waveguide is discussed in some detail by Baldwin and McInnea [4]. The parametric study of the properties of corrugated surfaces by Metzer and Peters [5] provides useful insights into the effects of ridge thickness, corrugation density, and surface resistivity. Work by these authors and others has been summarized by Clarricoats and Olver $|6|$. Studies nave shown that E-plane corrugated horns have substantially lower sidelobes than do smooth-wall horne of similar size, in some cases comparable to thove of conical corrugated horns. They also suggest that resistive losses may be lower than those of smooth-wall horns.

Corrugated horn are generally designed with two objectives: to achieve an antenna pattern with low sidelobes and to optimise the input match. One achieven these goals primarily by adjusting the depths of the corrugation slots to provide the desired field characteristics. Slots onequarter wave deep at the aperture cause the aperture-plane electric field to taper smoothly to zero at both the $E$-and $H$-plane walls; slots one-half wave in depth give rise to a field that matches the feld in uncorrugated rectangular guide, for a good match at the horn throat.

One must be careful to launch only the desired mode or modes in the corrugated horn. Most commonly, this is the $\mathrm{HE}_{12}$ rectangular mode (in the notation of $[6]$ ), whose transverse electric field component tapers smoothly to zero at the $H$-plane walls and also decreases smoothly toward the $E$-plane walls. In general, the presence of other modes causes higher sidelobe levels and a more irregular and more frequency-dependent radiation pattern. Among these undesired modes is the $H E_{11}$ slow-wave mode, whose field increases to a maximum at the $E$-plane walls, as well as various higher-order modes.

One can understand the effect of corrigations on the feld behavior by considering an $E$. plane corrugated rectangular waveguide (Fig. 1), whose properties resemble those of corrugated horns with moderate flare angles. The slots in the E-plane walls act as shorted segments of 
rectangular waveguide of length s, branching of from the main guide. Within a slot, waves are asoumed to propagate in the $\mathrm{TE}_{10}$ mode. (Note that the electric field within the slot is in the 2 direction, perpendicular to the ridges.) The $T E_{10}$ guide wavelength in the slot is $\lambda_{1}{ }^{1}$ given by the equation

$$
\lambda_{0}=\left(\frac{1}{\lambda^{2}}-\frac{1}{4 a^{2}}\right)^{-1 / 2},
$$

where $\lambda$ is the free-space wavelength. Reflections from the metal surface at the base of the slot create standing waves.

Boundary conditions for the allowed modes in the main guide are set by the requirements that the field components be continuous at the $E$-plane walls $(y= \pm b / 2)$, and that $E_{y}$ vanish at the amooth $H$-plane walls. If the alot depth $s$ is equal to $\lambda_{0} / 4, E_{y}$ and $H_{2}$ vanish at $y= \pm b / 2$, although a small $E$, component remains. In the $H E_{12}$ mode, $E_{y}$ decreases monotonically to zero at both the $E$ - and $H$-plane walls, yielding a radiation pattern with a well-defined peak and low sidelobes, whereas the value of $E_{y}$ for higher-order modes may go through several maxima and minima before vanishing at the walls. If $s=\lambda_{0} / 2$, the standing-wave condition at $y= \pm b / 2$ forces $E_{\text {s }}$ to vanish, creating boundary conditions equivalent to a conductive wall. In this case, the fields of the HE modes resemble those of the TE medes in uncorrugated waveguide. In particular, the $H E_{12}$ field distribution resembles that of the $T E_{10}$ mode in uncorrugated guide of width $a$ and height $b$.

The low-frequency cut-off for the $H E_{12}$ mode occurs when $\lambda_{1}=2 s+b$. To avoid excessive reflection at the transition from smooth to corrugated walls, one can include a short section of Gared imooth-wall waveguide at the horn throat in order to lower the $\mathrm{HE}_{12}$ cut-off frequency at the onset of corrugations. For good match over a moderate bandwidth, the $H E_{12}$ cuit-off frequency should be at least $20-30 \%$ below the minimum operating frequency. Reflections are further reduced if the first slot is made approximately $\lambda_{0} / 2$ deep and the subsequent slot depths are gradually decreased to $\lambda_{1} / 4$. An alternative form of smooth-to-corrugated transition-a gradual taper in the slot depths from 0 to $\lambda_{0} / 4$-is ruled out because it also launches the $H E_{11}$ slow-wave

'This is denoted $\lambda_{1}$ in the notation of $[4]$ and $[6]$. 
mode, which can propagate when $s \leq \lambda, / 4$. This mode is especially undesirable because the strong field at the $E$-plane walls causes high sidelobes.

\section{ANTENNA DESIGN AND CONSTRUCTION}

The antenna, shown in Fig. 2, can be divided for the purpose of description into four regions:

1) The smooth-wall throat section;

2) The intermediate corrugated section, whose slot depths taper from approximately $\lambda_{3} / 2$ near the throat to $\lambda_{0} / 4$ at the wide end;

3) The corrugated extension;

4) The quarter-wave traps at the mouth of the horn.

WR-650 waveguide at the throat $(16.5 \mathrm{~cm}$ by $8.3 \mathrm{~cm}$ cross-section) fiares out into the $16-\mathrm{cm}$ long smooth-walled section of the horn. Both the $E$ - and $H$-plane walls have a $19^{\circ}$ semi-flare angle; their ultimate dimensions are $27 \mathrm{~cm}$ and $19 \mathrm{~cm}$, respectively. This expanded section of smooth-walled guide lowers the $\mathrm{HE}_{12}$ cut-of frequency at the entrance to the corrugated section to approximately $980 \mathrm{MHz}$.

The intermediate corrugated section, $38 \mathrm{~cm}$ in length, serves as a transition from the smoothwalled section near the throat to the large section that comprises the main body of the horn. The two $E$-plane walls of this section contain corrugations whose ridge tops are coplanar with the walls of the smooth-walled section. The smooth $H$-plane walls continue, unbroken, from the previous section. Both the $E$ - and $H$-plane surfaces continue the $19^{\circ}$ flare of the smooth section. The first slot is $9.1 \mathrm{~cm}$ in depth, approximately $\lambda_{.} / 2$ at $1.74 \mathrm{GHz}$. The next 15 slots taper smoothly to a depth of $5.2 \mathrm{~cm}$, approximately $\lambda_{0} / 4$, at the far end of the section. The corrugations are spaced at $2.5-\mathrm{cm}$ incervals. Their density (approximately 7 to 9 corrugations per wavelength) yields acceptably low reflection withou: excessive resistive loss :5l.

The ridges are made from strips of $0.8-\mathrm{mm}$ aluminum sheet for reduced weight, ease of construction, and low ohmic loss. The strips are bent at right angles along their lengths and bolted to a flat aluminum backing sheet. The E-plane backing sheets open at a $13.5^{\circ}$ semi-flare 
angle in order to provide the taper in the slot depth. Lengths of aluminum angle running along the edges of the pyramid stiffen the structure and provide attachment surfaces for the $E$ - and $H$ plane walls. The walls are braced externally, and the $E$-plane ridges provide added stiffness. At their wide end, the E-plane walls are $53 \mathrm{~cm}$ across, while the maximum width of the $H$-plane walls is $45 \mathrm{~cm}$.

The extension is a continuation of the previous corrugated section, with the same $19^{\circ}$ semi-flare angle and a slot depth of $5.2 \mathrm{~cm}$ throughout the $145-\mathrm{cm}$ length of the section. The dimensions of horn mouth are $149 \mathrm{~cm}$ by $145 \mathrm{~cm}$. The choice of opening angle and aperture dimensions causes the phase to vary by more than $180^{\circ}$ across the aperture, for a beam pattern whose main lobe is wider than that of a diffraction-limited horn but less frequency-dependent.

To further reduce the sidelobe and backlobe response, we have installed quarter-wave-deep choke slots in the aperture plane around the periphery of the horn, a technique that has been shown to work well for circular antennas $[7,8]$. Along each edge of the aperture are five slots, each one $5.2 \mathrm{~cm}$ deep and $2.5 \mathrm{~cm}$ wide, made also of folded sheet metal bolted to a backing sheet. No attempt has been made to optimize either the number or the width of these slots, since the design used here provides sufficient backlobe suppression for our needs.

\section{REFLECTION}

The return loss of the horn, measured by means of a slotted line, is plotted in Fig. 3 . Between 1.3 and $1.7 \mathrm{GHz}$, the return loss is $-25 \mathrm{~dB}$ or lower. Removal of the antenna extension increases the reflected signal by $\sim 1 \mathrm{~dB}$ or less, an indication not only of the fact that very little reflection occurs within the extension but also of the good match between the mouth of the intermediate corrugated section and free space.

\section{RADIATION PATTERN}

We have measured the antenna's radiation pattern on a roof-top test range, with a $24-m$ separation between the source antenna and the test antenna. Scattering from near-field objects in the test range prevents accurate measurements at angles greater than $\sim 150^{\circ}$, but measurements 
of other rectangular corrugated horns indicate that the response from $150^{\circ}$ to $180^{\circ}$ is likely to be similar to the pattern between $90^{\circ}$ and $150^{\circ}[3]$. To evaluate the contrit ition from the quarterwave choke slots at the aperture, we have performed measurements both with and without them. Figure 4 shows the $E$-and $H$-plane patterns at $1.3,1.5$, and $1.7 \mathrm{GHz}$. The shape of the $E$-plane main lobe is quite similar at all three frequencies, with a half-power beamwidth that varies from $21^{\circ}$ at $1.3 \mathrm{GH}_{3}$ to $18^{\circ}$ at $1.7 \mathrm{GHz}$. The antenna response between $90^{\circ}$ and $150^{\circ}$ is below $-55 \mathrm{~dB}$ with the choke slots in place and below $-.50 \mathrm{~dB}$ without them. The unexpectedly high sidelobes at 1.7 GHz may be evidence of a secondary mode at this frequency.

The $H$-plane main lobe is narrower and somewhat more frequency-dependent than its $E$-plane counterpart. The half-power beamwidth varies from $17^{\circ}$ at $1.3 \mathrm{GHz}$ to $13^{\circ}$ at $1.7 \mathrm{GHz}$. The $H$-plane sidelobes show structure at the -55 to $-60 \mathrm{~dB}$ level from $90^{\circ}$ to $150^{\circ}$. The relatively high sidelobes at $1.7 \mathrm{GHz}$ again suggest the presence of a secondary mode. The patterns are approximately what one would expect from an aperture field with a cosine distribution in the $E$. and $H$-planes, and a phase error given by the dimensions of the antenna.

The effect of the quarter-wave choke slots is shown in Fig. 4. The E-plane antenna response at angles greater than $\sim 75^{\circ}$ is reduced by as much $210 \mathrm{~dB}$. The $H$-plane choke slots have no significant effect; sidelobe and backlobe levels are virtually the same with and without them.

Scattering by objects in the test range prevented us from making accurate far-field measurements of the cross-polarized response, although we were able to make rough measurements of the response in the aperture plane. The ratio of compolarized to cross-polarized response meseured at the aperture varied from $<10 \mathrm{~dB}$ near the $H$-plane walls to $>35 \mathrm{~dB}$ near the center of the aperture.

\section{CONCLUSIONS}

We have shown that rectangular corrugated horn antennas with good sidelobe suppression over at least a $25 \%$ bandwidth can be made from sheet metal, using simple techniques. Quarterwave choke slots at the aperture flange, also fabricated from sheet metal, provide another 5 to 
$10 \mathrm{~dB}$ of sidelobe and backlobe suppression in the $E$-plane; greater backlobe suppression may be obtainable with a different choice of slot width and number. The techniques described here permit one to build corrugated horns to operate in a frequency range where such horns constructed by conventional techniques would not be practical.

\section{ACKNOWLEDGEMENT}

We would like to acknowledge the assistance of Marco Berssnelli, Giovanni De Amici, Al Kogut, and Carol Stanton in the construction and testing of the antenna. We would also like to thank William T. Lum and David Williams of the University of California Radio Astronomy Laboratory for their advice and assistance. This work was supported by National Science Foundation grant AST.8406187 and by the Department of Energy under Contract DE-AC03. 76 SF00098.

\section{REFERENCES}

[1] G. F. Smoot et al., "Low-frequency measurements of the cosmic background radiation spectrum," Ap. J., vol. 291, Pp. L23-L27, April 1985.

(2) M. A. Janssen, S. M. Bednarczyk, S. Gulkis, H. W. Marlin, and G. F. Smoot, "Pattern measurement of a low-sidelobe horn antenna," IEEE Trans. Antennas Propagat., vol. AP-27, pp. 551-555, July 1979.

(3) R. E. Lawrie and L. Peters, Jr,, “Modifications of horn antennas for low sidelobe levels," IEEE Trans. Antennas Propagat., vol. AP-14, pp. 605-610, Sept. 1966.

4) R. Baldwin and P. A. McInnes, "Corrugated rectangular horns for use as microwave feeds," Proc. IEE, vol. 122, pp. 465-469, May 1975.

(5) C. A. Metzner and L. Peters, Jr., "Properties of cutoff corr'ıgated surfaces for corrugated horn design," IEEE Trans. Antennas Propagat., vol. AP-22, pp. 191-196, Mar. 1974.

[6] P. J. B. Clarricoats and A. D. Olver, Corrugated Horns for Microwave Antennas, London: Peter Peregrinus, 1984. 
[7] G. A. Hockham, "Investigation of a $90^{\circ}$ corrugated horn," Electron. Lett., vol. 12, pp. 199-20i, Apr. 1976.

[8] R. Wohlleben, H. Mattes, and O. Lochner, "Simple small primary feed for large opening angles and high aperture efficiency," Electron. Lett., vol. 8, pp. 474-6, Sept. 1972. 
Figure 1: E-plane corrugated rectangular waveguide.

Figure 2: $\not K$-plane cutaway view of rectangular corrugated horn.

Figure 3: Corrugated antenna return loss. Points indicate measured values.

Figure 4: Measured E- and H-plane patterns for rectangular corrugated horn. Successive patterns offset by $20 \mathrm{~dB}$. 
Page Clo

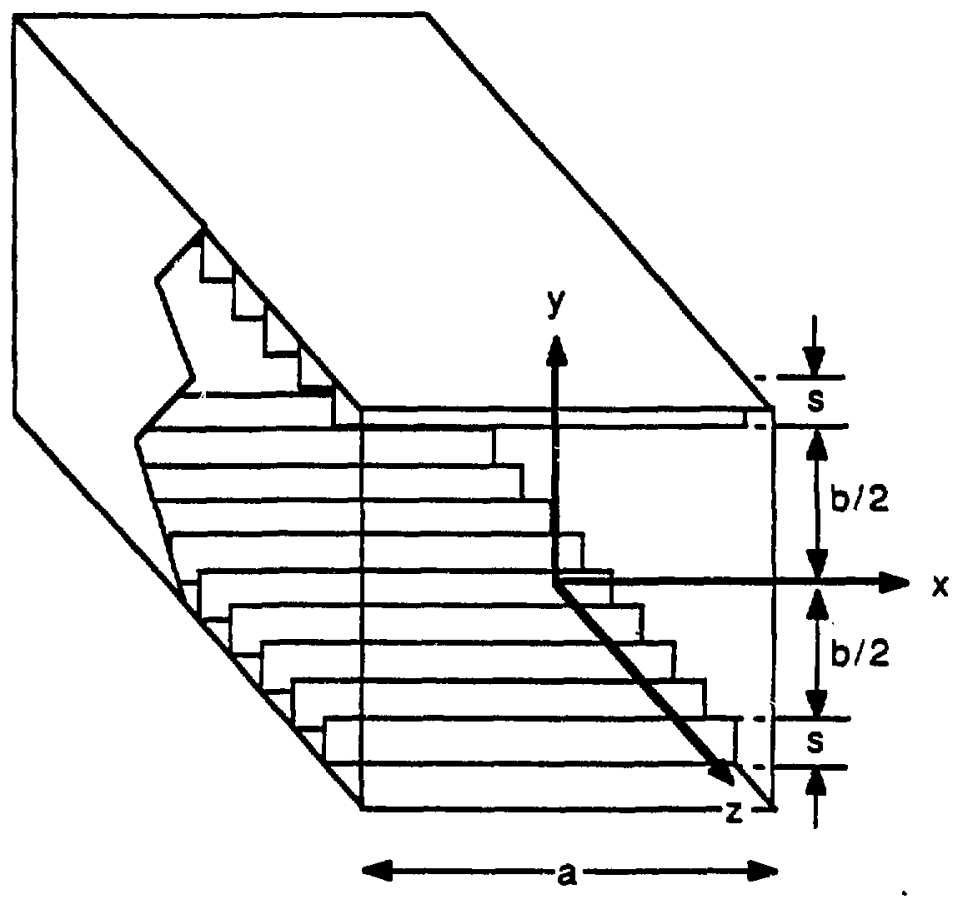

Figure 1: $E$-plane corrugated rectangular waveguide. 
Figure 2: $H$-plane cutaway view of rectangular corrugated horn.

\section{(4)}

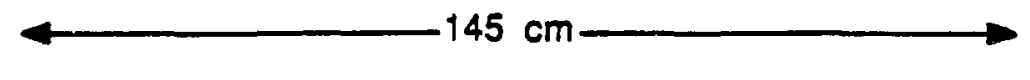

\section{Ш世}

(3)
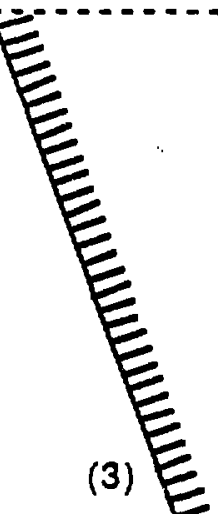

(2)
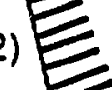

$38 \mathrm{~cm}$

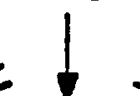

(1)

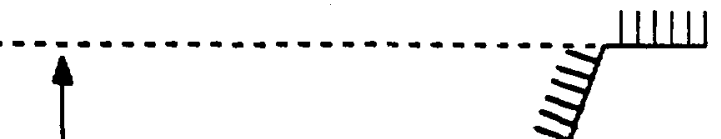




\section{Return Loss (dB)}
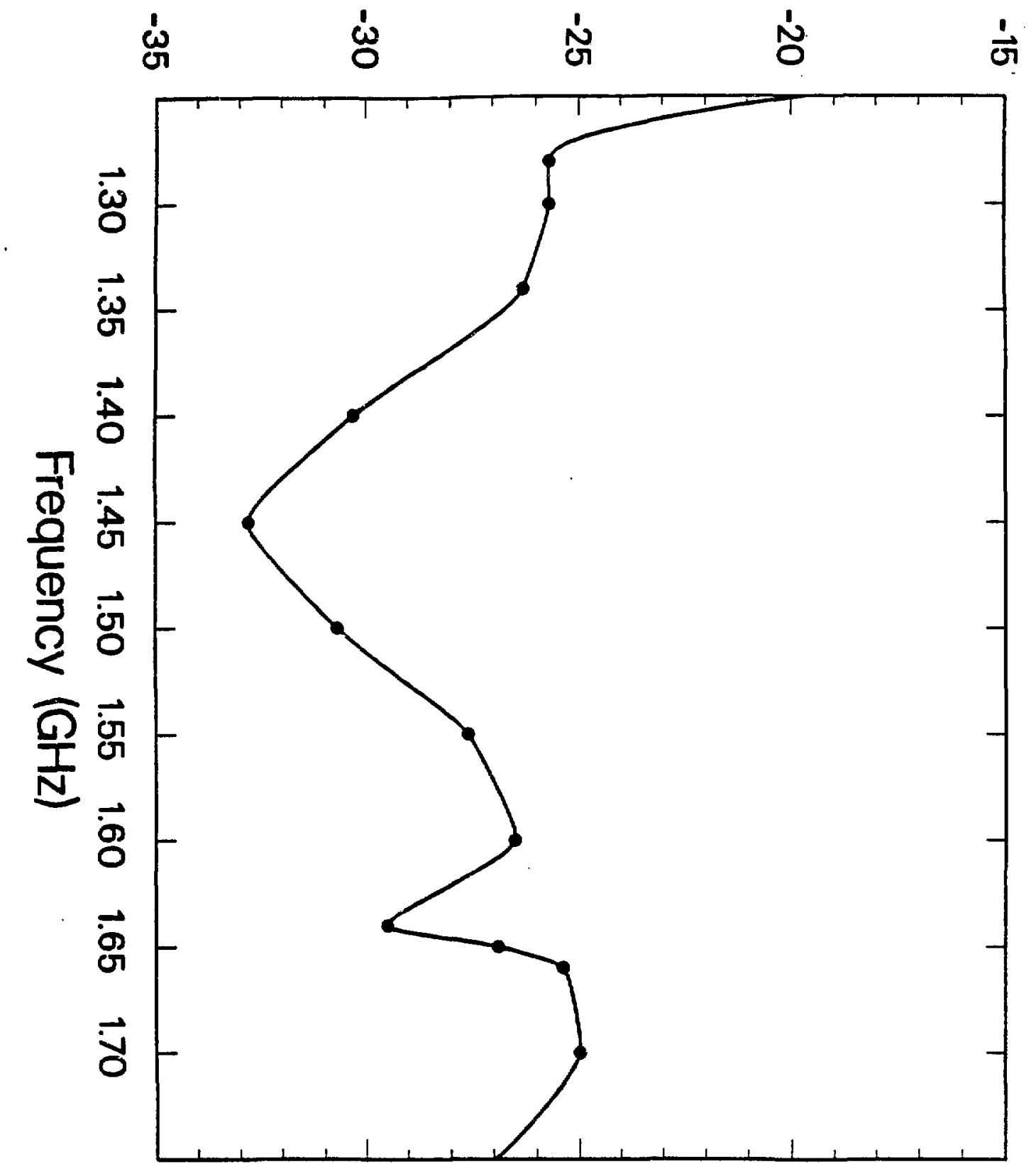

Figure 3: Corrugated antenna return loss. Points indicate measured values 


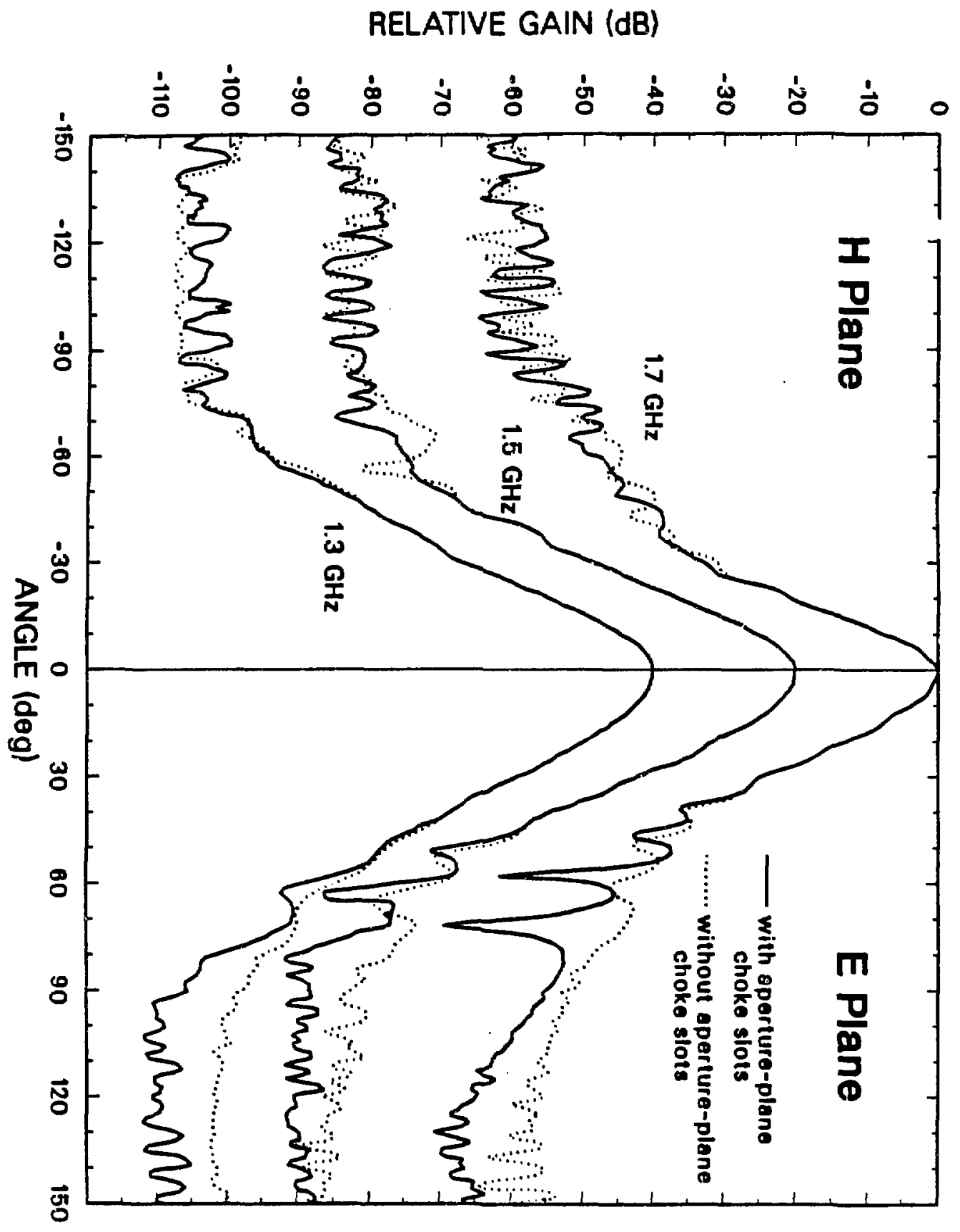

Figure 4: Measured E- and H-plane patterns for rectangular corrugated horn. Successive patcerng offset by $20 \mathrm{~dB}$. 